\title{
Study of $\Lambda_{c} \Lambda_{c}$ dibaryon and $\Lambda_{c} \bar{\Lambda}_{c}$ baryonium states via QCD sum rules
}

\author{
Xiu-Wu Wang ${ }^{1,2} *$ Zhi-Gang Wang $\left.{ }^{1}\right\rfloor^{\dagger}$ and Guo-liang $\mathrm{Yu}^{1}$ \\ 1 School of Mathematics and Physics, North China Electric power university, \\ Baoding, 071003, People's Republic of China \\ 2 School of Nuclear Science and Engineering, \\ North China Electric power University, Beijing, 102206, People's Republic of China
}

In this article, we construct the six-quark currents with the $J^{P}=0^{+}, 0^{-}, 1^{+}$and $1^{-}$to study the $\Lambda_{c} \Lambda_{c}$ dibaryon and $\Lambda_{c} \bar{\Lambda}_{c}$ baryonium states via QCD sum rules. We consider the vacuum condensates up to dimension 16 and truncation of the order $\mathcal{O}\left(\alpha_{s}^{k}\right)$ with $k \leq 3$. The predicted masses are $5.11_{-0.12}^{+0.15} \mathrm{GeV}, 4.66_{-0.06}^{+0.10} \mathrm{GeV}, 4.99_{-0.09}^{+0.10} \mathrm{GeV} 4.68_{-0.08}^{+0.08} \mathrm{GeV}$ for the $J^{P}=0^{+}, 0^{-}, 1^{+}$and $1^{-}$ states, respectively, which can be confronted to the experimental data in the future considering the high integrated luminosity at the center-of-mass energy about $4.8 \mathrm{GeV}$ at the BESIII. We find the terms with $\frac{3}{2}<k \leq 3$ do play a tiny role, and we can ignore these terms safely in the QCD sum rules.

Keywords: dibaryon states; QCD sum rules

PACS numbers: 12.39.Mk, 12.38.Lg

\section{Introduction}

In 2003, $X(3872)$ was discovered by the Belle collaboration [1], later on, this state was confirmed by the BABAR collaboration [2]. After that, many charmonium-like and bottomonium-like states were observed. Due to the extraordinary properties of these exotic $X, Y$ and $Z$ states, it is hard to embed them into the conventional charmonium spectrum. The charged charmonium-like states are good candidates for the multiquark states, for example, tetraquark states and molecular states [3]. Among these exotic states, the $\Lambda_{c} \bar{\Lambda}_{c}$ baryonium state was introduced to interpret the decays of the $Y(4260)$ [4, 5]. In 2006, the $X$ (1835) was tentatively interpreted as a proton-antiproton baryonium state based on the QCD sum rules [6]. Interestingly, the BESIII collaboration searched for the strange cousin of the $Z_{c}(3885)$ in the process $e^{+}+e^{-} \rightarrow K^{+}\left(D_{s}^{-} D^{* 0}+D_{s}^{*-} D^{0}\right)[7]$, they observed the strongest signal at the center-of-mass energy $\sqrt{s}=4.681 \mathrm{GeV}$, but they did not see any evidence of exotic states in the $D_{s}^{*-} D_{s 2}^{*}(2573)^{+}$mass spectrum.

The dibaryon state is composed by two baryons. The well-known dibaryon state is the deuteron, the bound state of a proton and neutron with binding energy $E_{B}=2.225 \mathrm{MeV}$. In 1994, the $H$-dibaryon state was studied with the QCD sum rules [8], thereafter, applying the QCD sum rules to study the

\footnotetext{
*Electronic address: wangxiuwu2020@163.com

${ }^{\dagger}$ Electronic address: zgwang@aliyun.com
} 
dibaryon and baryonium states has triggered much attention. In Ref. [9], the masses of the $d^{*}(2380)$ et al were calculated using the $\Delta \Delta$-like six-quark local interpolating currents. In Ref.[10], the scalar and axialvector $\Xi_{c c} \Sigma_{c}$ dibaryon states were studied using the color-singlet-color-singlet type interpolating currents. And very recently, the hidden-bottom and hidden-charm hexaquark molecular states with the quantum numbers $J^{P C}=0^{++}, 0^{-+}, 1^{++}$, and $1^{--}$have been studied in the framework of the QCD sum rules [11]. It is proposed via the Bethe-Salpeter equation that the $\Lambda_{c} \bar{\Lambda}_{c}$ baryonium states should contain at least two states: the $\Lambda_{c} \bar{\Lambda}_{c}$ molecular state and another one with mass around $4.65 \mathrm{GeV}$ for the thresholds up to $4.7 \mathrm{GeV}[12]$. But the masses of the $\Lambda_{c} \bar{\Lambda}_{c}$ baryonium states in Ref.[11] are all above $4.7 \mathrm{GeV}$.

The QCD sum rules is a successful method in studying the hadronic properties 13 25]. It could be used to calculate the masses of hadrons, decay constants, hadronic form-factors, coupling constants, etc [26, 27]. This method has been applied extensively to study the hidden-charm (bottom) tetraquark (molecular) states [13 23, 28 30] and pentaquark (molecular) states [31, 32]. In this paper, we construct four six-quark local interpolating currents to study both the $\Lambda_{c} \Lambda_{c}$ dibaryon and $\Lambda_{c} \bar{\Lambda}_{c}$ baryonium states with the $J^{P}=0^{+}, 0^{-}, 1^{+}$and $1^{-}$, respectively.

The article is arranged as follows: we derive the QCD sum rules for the masses and pole residues of the dibaryon and baryonium states in Sect.2; we present the numerical results and discussions in Sect.3; Sect.4 is reserved for our conclusion.

\section{QCD sum rules for the dibaryon and baryonium states}

We begin our calculation from the following two-point correlation functions,

$$
\begin{aligned}
& \Pi_{1}(p)=i \int d^{4} x e^{i p \cdot x}\left\langle 0\left|J_{1}(x) J_{1}^{\dagger}(0)\right| 0\right\rangle, \\
& \Pi_{2}(p)=i \int d^{4} x e^{i p \cdot x}\left\langle 0\left|J_{2}(x) J_{2}^{\dagger}(0)\right| 0\right\rangle, \\
& \Pi_{3 ; \mu \nu}(p)=i \int d^{4} x e^{i p \cdot x}\left\langle 0\left|J_{3, \mu}(x) J_{3, \nu}^{\dagger}(0)\right| 0\right\rangle, \\
& \Pi_{4 ; \mu \nu}(p)=i \int d^{4} x e^{i p \cdot x}\left\langle 0\left|J_{4, \mu}(x) J_{4, \nu}^{\dagger}(0)\right| 0\right\rangle,
\end{aligned}
$$

where

$$
\begin{aligned}
& J_{1}(x)=J_{\Lambda}^{T}(x) C \gamma_{5} J_{\Lambda}(x), \\
& J_{2}(x)=\bar{J}_{\Lambda}(x) i \gamma_{5} J_{\Lambda}(x), \\
& J_{3, \mu}(x)=\bar{J}_{\Lambda}(x) \gamma_{5} \gamma_{\mu} J_{\Lambda}(x), \\
& J_{4, \mu}(x)=\bar{J}_{\Lambda}(x) \gamma_{\mu} J_{\Lambda}(x),
\end{aligned}
$$

$J_{\Lambda}(x)=\varepsilon^{i j k} u^{i T}(x) C \gamma_{5} d^{j}(x) c^{k}(x)$, the superscripts $i, j, k$ are color indices and $C$ represents the charge 
conjugation matrix. Under parity transformation $\widehat{P}$, the four currents satisfy the following equations,

$$
\begin{aligned}
& \widehat{P} J_{1}(x) \widehat{P}^{-1}=J_{1}(\widetilde{x}), \\
& \widehat{P} J_{2}(x) \widehat{P}^{-1}=-J_{2}(\widetilde{x}), \\
& \widehat{P} J_{3, \mu}(x) \widehat{P}^{-1}=-J_{3}^{\mu}(\widetilde{x}), \\
& \widehat{P} J_{4, \mu}(x) \widehat{P}^{-1}=J_{4}^{\mu}(\widetilde{x}),
\end{aligned}
$$

where $x^{\mu}=\left(x^{0}, x^{1}, x^{2}, x^{3}\right)$ and $\widetilde{x}^{\mu}=\left(x^{0},-x^{1},-x^{2},-x^{3}\right)$. As the phenomenological expressions of the correlation functions are concerned, we insert a complete set of intermediate hadronic states with the same quantum numbers as the current operators $J_{1,2,3,4}(x)$ into the correlation functions and get,

$$
\begin{aligned}
& \Pi_{1,2}(p)=\frac{\lambda_{1,2}^{2}}{M_{1,2}^{2}-p^{2}}+\ldots \\
& \Pi_{3,4 ; \mu \nu}(p)=A_{3,4}(p)\left(-g_{\mu \nu}+\frac{p_{\mu} p_{\nu}}{p^{2}}\right)+\ldots
\end{aligned}
$$

where

$$
\begin{aligned}
& A_{3,4}(p)=\frac{\lambda_{3,4}^{2}}{M_{3,4}^{2}-p^{2}}+\ldots, \\
& \left\langle 0\left|J_{1,2}(0)\right| Z_{1,2}(p)\right\rangle=\lambda_{1,2}, \\
& \left\langle 0\left|J_{3,4 ; \mu}(0)\right| Z_{3,4}(p)\right\rangle=\lambda_{3,4} \epsilon_{\mu},
\end{aligned}
$$

$\left|Z_{1,2,3,4}\right\rangle$ denote the ground states and $\lambda_{1,2,3,4}$ are the pole residues, which show the couplings of the currents $J_{1,2,3,4}$ to the molecular states, the $\epsilon_{\mu}$ are the polarization vectors.

We apply the Wick theorem to contract the quark fields and acquire the expressions of the correlation functions in terms of full quark propagators,

$$
\begin{aligned}
& \Pi_{1}(p)=2 \varepsilon^{i j k} \varepsilon^{l m n} \varepsilon^{i^{\prime} j^{\prime} k^{\prime}} \varepsilon^{l^{\prime} m^{\prime} n^{\prime}} i \int d^{4} x e^{i p \cdot x} \\
& \left\{-\operatorname{Tr}\left\{\gamma_{5} B^{k k^{\prime}}(x) \gamma_{5} C B^{n n^{\prime} T}(x) C\right\} \operatorname{Tr}\left\{\gamma_{5} Q^{j j^{\prime}}(x) \gamma_{5} C Q^{i i^{\prime} T}(x) C\right\} \operatorname{Tr}\left\{\gamma_{5} Q^{m m^{\prime}}(x) \gamma_{5} C Q^{l l^{\prime} T}(x) C\right\}\right. \\
& -\operatorname{Tr}\left\{\gamma_{5} B^{k n^{\prime}}(x) \gamma_{5} C B^{n k^{\prime} T}(x) C\right\} \operatorname{Tr}\left\{\gamma_{5} Q^{j j^{\prime}}(x) \gamma_{5} C Q^{i i^{\prime} T}(x) C\right\} \operatorname{Tr}\left\{\gamma_{5} Q^{m m^{\prime}}(x) \gamma_{5} C Q^{l l^{\prime} T}(x) C\right\} \\
& +\operatorname{Tr}\left\{\gamma_{5} B^{k k^{\prime}}(x) \gamma_{5} C B^{n n^{\prime} T}(x) C\right\} \operatorname{Tr}\left\{\gamma_{5} Q^{l i^{\prime}}(x) \gamma_{5} C Q^{j j^{\prime} T}(x) C \gamma_{5} Q^{i l^{\prime}}(x) \gamma_{5} C Q^{m m^{\prime} T}(x) C\right\} \\
& \left.+\operatorname{Tr}\left\{\gamma_{5} B^{k k^{\prime}}(x) \gamma_{5} C B^{n n^{\prime} T}(x) C\right\} \operatorname{Tr}\left\{\gamma_{5} Q^{i i^{\prime}}(x) \gamma_{5} C Q^{m j^{\prime} T}(x) C \gamma_{5} Q^{l l^{\prime}}(x) \gamma_{5} C Q^{j m^{\prime} T}(x) C\right\}\right\}, \\
& \Pi_{2}(p)=\varepsilon^{i j k} \varepsilon^{l m n} \varepsilon^{i^{\prime} j^{\prime} k^{\prime}} \varepsilon^{l^{\prime} m^{\prime} n^{\prime}} i \int d^{4} x e^{i p \cdot x} \\
& \operatorname{Tr}\left\{\gamma_{5} B^{n n^{\prime}}(x) \gamma_{5} B^{k^{\prime} k}(-x)\right\} \operatorname{Tr}\left\{\gamma_{5} Q^{m m^{\prime}}(x) \gamma_{5} C Q^{l l^{\prime} T}(x) C\right\} \operatorname{Tr}\left\{\gamma_{5} Q^{j^{\prime} j}(-x) \gamma_{5} C Q^{i^{\prime} i T}(-x) C\right\}, \\
& \Pi_{3 ; \mu \nu}(p)=-\varepsilon^{i j k} \varepsilon^{l m n} \varepsilon^{i^{\prime} j^{\prime} k^{\prime}} \varepsilon^{l^{\prime} m^{\prime} n^{\prime}} i \int d^{4} x e^{i p \cdot x} \\
& \operatorname{Tr}\left\{\gamma_{5} \gamma_{\mu} B^{k k^{\prime}}(x) \gamma_{5} \gamma_{\nu} B^{n^{\prime} n}(-x)\right\} \operatorname{Tr}\left\{\gamma_{5} Q^{j j^{\prime}}(x) \gamma_{5} C Q^{i i^{\prime} T}(x) C\right\} \operatorname{Tr}\left\{\gamma_{5} Q^{m^{\prime} m}(-x) \gamma_{5} C Q^{l^{\prime} l T}(-x) C\right\}
\end{aligned}
$$




$$
\begin{aligned}
& \Pi_{4 ; \mu \nu}(p)=-\varepsilon^{i j k} \varepsilon^{l m n} \varepsilon^{i^{\prime} j^{\prime} k^{\prime}} \varepsilon^{l^{\prime} m^{\prime} n^{\prime}} i \int d^{4} x e^{i p \cdot x} \\
& \operatorname{Tr}\left\{\gamma_{\mu} B^{k k^{\prime}}(x) \gamma_{\nu} B^{n^{\prime} n}(-x)\right\} \operatorname{Tr}\left\{\gamma_{5} Q^{j j^{\prime}}(x) \gamma_{5} C Q^{i i^{\prime} T}(x) C\right\} \operatorname{Tr}\left\{\gamma_{5} Q^{m^{\prime} m}(-x) \gamma_{5} C Q^{l^{\prime} l T}(-x) C\right\},
\end{aligned}
$$

where $Q^{a b}(x)$ and $B^{a b}(x)$ are the full light and heavy quark propagators respectively, and they can be written as,

$$
\begin{gathered}
Q^{a b}(x)=\quad \frac{i \not \delta^{a b}}{2 \pi^{2} x^{4}}-\frac{\delta^{a b}}{12}\langle\bar{q} q\rangle-\frac{\delta^{a b} x^{2}}{192}\left\langle\bar{q} g_{s} \sigma G q\right\rangle-\frac{i \delta^{a b} x^{2} \not g_{s}^{2}\langle\bar{q} q\rangle^{2}}{7776}-t_{a b}^{n}\left(\not \sigma^{\alpha \beta}+\sigma^{\alpha \beta} \not x\right) \frac{i}{32 \pi^{2} x^{2}} g_{s} G_{\alpha \beta}^{n} \\
-\frac{\delta^{a b} x^{4}\langle\bar{q} q\rangle\langle G G\rangle}{27648}-\frac{1}{8}\left\langle\bar{q}^{b} \sigma^{\alpha \beta} q^{a}\right\rangle \sigma_{\alpha \beta}-\frac{1}{4}\left\langle\bar{q}^{b} \gamma_{\mu} q^{a}\right\rangle \gamma^{\mu}+\cdots, \\
B_{a b}(x)=\frac{i}{(2 \pi)^{4}} \int d^{4} k e^{-i k \cdot x}\left\{\frac{\delta_{a b}}{\not k-m_{c}}-\frac{g_{s} G_{\alpha \beta}^{h} t_{a b}^{h}}{4} \frac{\sigma^{\alpha \beta}\left(\not k+m_{c}\right)+\left(\not k+m_{c}\right) \sigma^{\alpha \beta}}{\left(k^{2}-m_{c}^{2}\right)^{2}}\right. \\
+\frac{g_{s} D_{\alpha} G_{\beta \lambda}^{h} t_{a b}^{h}\left(f^{\lambda \beta \alpha}+f^{\lambda \alpha \beta}\right)}{3\left(k^{2}-m_{c}^{2}\right)^{4}}-\frac{g_{s}^{2}\left(t^{h} t^{r}\right)_{a b} G_{\alpha \beta}^{h} G_{\mu \nu}^{r}\left(f^{\alpha \beta \mu \nu}+f^{\alpha \mu \beta \nu}+f^{\alpha \mu \nu \beta}\right)}{4\left(k^{2}-m_{c}^{2}\right)^{5}} \\
\left.\frac{\langle G G G\rangle}{48\left(k^{2}-m_{c}^{2}\right)^{6}}\left(\not k+m_{c}\right)\left[\not k\left(k^{2}-3 m_{c}^{2}\right)+2 m_{c}\left(2 k^{2}-m_{c}^{2}\right)\right]\left(\not k+m_{c}\right)+\cdots\right\}, \\
f^{\lambda \alpha \beta}=\left(\not k+m_{c}\right) \gamma^{\lambda}\left(\not k+m_{c}\right) \gamma^{\alpha}\left(\not k+m_{c}\right) \gamma^{\beta}\left(\not k+m_{c}\right), \\
f^{\alpha \beta \mu \nu}=\left(\not k+m_{c}\right) \gamma^{\alpha}\left(\not k+m_{c}\right) \gamma^{\beta}\left(\not k+m_{c}\right) \gamma^{\mu}\left(\not k+m_{c}\right) \gamma^{\nu}\left(\not k+m_{c}\right),
\end{gathered}
$$

where $t^{n}=\frac{\lambda^{n}}{2}, \lambda^{n}$ is the Gell-Mann matrix and $D_{\alpha}=\partial_{\alpha}-i g_{s} G_{\alpha}^{h} t^{h}$ [27, 28, 33]. Here, we consider the term $\left\langle\bar{q}^{b} \sigma^{\alpha \beta} q^{a}\right\rangle\left(\left\langle\bar{q}^{b} \gamma^{\mu} q^{a}\right\rangle\right)$ which is derived from Fierz rearrangement of the quark-antiquark pair $\left\langle q^{a} \bar{q}^{b}\right\rangle$, it is used to absorb the gluons emitted from other quark lines to form the mixed condensates $\left\langle\bar{q} g_{s} \sigma G q\right\rangle,\left\langle\bar{q} g_{s} \sigma G q\right\rangle^{2}$ and $\left\langle\bar{q} g_{s} \sigma G q\right\rangle^{3}$, respectively (the four-quark condensate $g_{s}^{2}\langle\bar{q} q\rangle^{2}$ ) [28]. For the light quark propagator, we calculate the integrals in the coordinate space, as for the heavy quark counterpart, we consider the momentum space.

Now, let us draw the Feynman diagrams for the leading order contribution of these correlation functions, see Fig.1, there are four light quark lines and two heavy quark lines. Considering each heavy quark line emits a gluon and each light quark line contributes quark-antiquark pair, we obtain the quark-gluon operator $g_{s} G_{\alpha \beta} g_{s} G_{\eta \tau} \bar{q} q \bar{q} q \bar{q} q \bar{q} q$ with dimension 16 . This operator leads to the vacuum condensates $\left\langle\frac{\alpha_{s}}{\pi} G G\right\rangle\langle\bar{q} q\rangle^{4}$ and $\left\langle\bar{q} g_{s} \sigma G q\right\rangle^{2}\langle\bar{q} q\rangle^{2}$. In order to find the exact contributions of different kinds of the vacuum condensates, we choose the $J_{1}(x)$ for detailed calculations and consider all kinds of the vacuum condensates up to dimension 16 and take the truncation of the order $\mathcal{O}\left(\alpha_{s}^{k}\right)$ with $k \leq 3$. The considered terms include $\left\langle\frac{\alpha_{s}}{\pi} G G\right\rangle,\langle\bar{q} q\rangle^{2},\langle\bar{\psi} \psi\rangle^{2}=\sum_{u, s, d}\langle\bar{q} q\rangle^{2},\left\langle g_{s}^{3} f G G G\right\rangle,\left\langle\bar{q} g_{s} \sigma G q\right\rangle\langle\bar{q} q\rangle$, $\left\langle\frac{\alpha_{s}}{\pi} G G\right\rangle^{2},\left\langle\bar{q} g_{s} \sigma G q\right\rangle^{2},\left\langle\frac{\alpha_{s}}{\pi} G G\right\rangle\langle\bar{q} q\rangle^{2},\langle\bar{q} q\rangle^{4},\left\langle\bar{q} g_{s} \sigma G q\right\rangle\left\langle\frac{\alpha_{s}}{\pi} G G\right\rangle\langle\bar{q} q\rangle,\left\langle g_{s}^{3} f G G G\right\rangle^{2},\left\langle\frac{\alpha_{s}}{\pi} G G\right\rangle^{3},\langle\bar{q} q\rangle^{2}\langle\bar{\psi} \psi\rangle^{2}$, $\left\langle\bar{q} g_{s} \sigma G q\right\rangle\langle\bar{q} q\rangle^{3},\langle\bar{q} q\rangle\left\langle\bar{q} g_{s} \sigma G q\right\rangle\langle\bar{\psi} \psi\rangle^{2},\left\langle\frac{\alpha_{s}}{\pi} G G\right\rangle^{2}\langle\bar{q} q\rangle^{2},\left\langle\bar{q} g_{s} \sigma G q\right\rangle^{2}\left\langle\frac{\alpha_{s}}{\pi} G G\right\rangle,\left\langle\bar{q} g_{s} \sigma G q\right\rangle^{2}\langle\bar{q} q\rangle^{2},\left\langle\frac{\alpha_{s}}{\pi} G G\right\rangle\langle\bar{q} q\rangle^{4}$, 


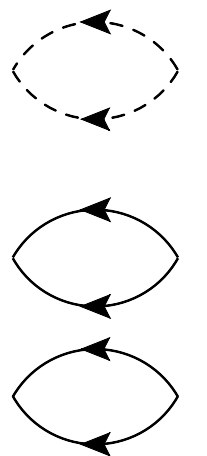

(1)
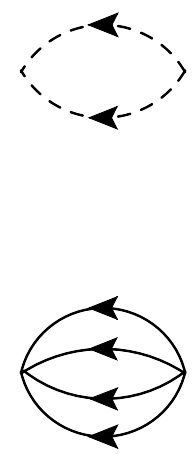

(2)
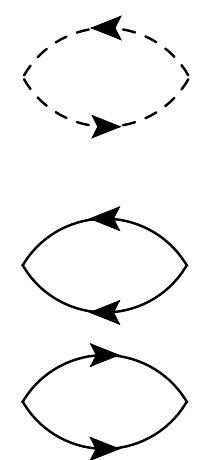

(3)

FIG. 1: The Feynman diagrams (1) and (2) (diagram (3)) are the lowest order contributions for the current $J_{1}$ (currents $J_{2,3,4}$ ), where the solid and dashed lines represent the light and heavy quark propagators respectively.

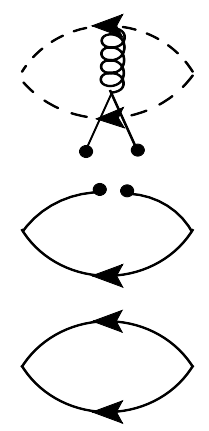

(1)

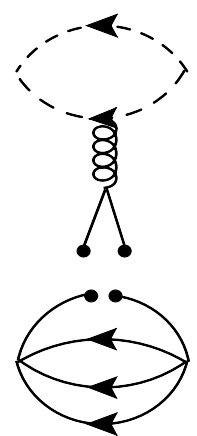

(2)
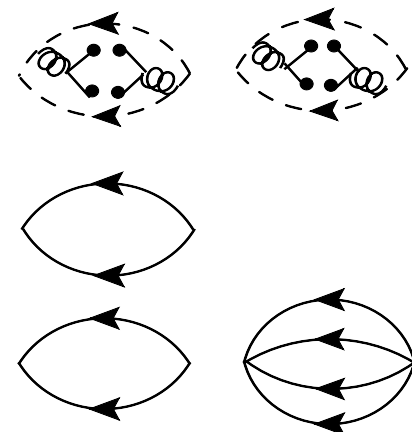

(3)

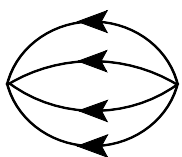

(4)

FIG. 2: The Feynman diagrams contribute to the four-quark condensates, where (1)(2) for $\langle\bar{q} q\rangle^{2}$ and $(3)(4)$ for $\langle\bar{\psi} \psi\rangle^{2}$, respectively.

$\left\langle\bar{q} g_{s} \sigma G q\right\rangle\left\langle\frac{\alpha_{s}}{\pi} G G\right\rangle^{2}\langle\bar{q} q\rangle$ and $\left\langle\bar{q} g_{s} \sigma G q\right\rangle^{2}\langle\bar{\psi} \psi\rangle^{2}$. The vacuum condensates $\langle\bar{q} q\rangle,\left\langle\bar{q} g_{s} \sigma G q\right\rangle$ and $\left\langle\bar{q} g_{s} \sigma G q\right\rangle^{3}$ have no contribution for all the four currents. From the numerical results, we find that those terms with $k \geq \frac{3}{2}$ do play a tiny role. So, for the currents $J_{2,3,4}$, it is safe for us to take account of only the terms $\left\langle\frac{\alpha_{s}}{\pi} G G\right\rangle,\langle\bar{q} q\rangle^{2},\left\langle\bar{q} g_{s} \sigma G q\right\rangle\langle\bar{q} q\rangle,\left\langle\bar{q} g_{s} \sigma G q\right\rangle^{2},\left\langle\frac{\alpha_{s}}{\pi} G G\right\rangle\langle\bar{q} q\rangle^{2},\langle\bar{q} q\rangle^{4},\left\langle\bar{q} g_{s} \sigma G q\right\rangle\langle\bar{q} q\rangle^{3},\left\langle\bar{q} g_{s} \sigma G q\right\rangle^{2}\langle\bar{q} q\rangle^{2}$ and $\left\langle\frac{\alpha_{s}}{\pi} G G\right\rangle\langle\bar{q} q\rangle^{4}$.

For the current $J_{1}$, we take into account of the four-quark condensates, whose typical Feynman diagrams are shown in Fig.2; the three-gluon condensates, whose typical Feynman diagrams are shown in Fig.3. We also take into consideration of the $\left\langle\frac{\alpha_{s}}{\pi} G G\right\rangle^{2}$ of the order $\mathcal{O}\left(\alpha_{s}^{2}\right)$, the corresponding Feynman diagrams are shown in Fig.4 and Fig.5. The typical terms with the highest truncation $k=3$ are the vacuum condensates $\left\langle\frac{\alpha_{s}}{\pi} G G\right\rangle^{3}$ and $\left\langle g_{s}^{3} f G G G\right\rangle^{2}$. Parts of the Feynman diagrams are shown in Fig.6, one can draw other diagrams in a similar way as displayed. 


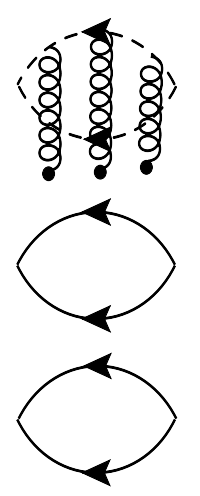

(1)

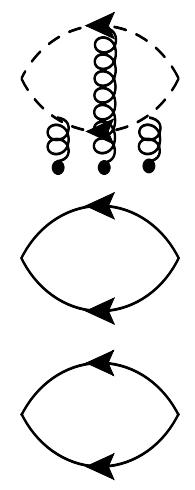

(2)

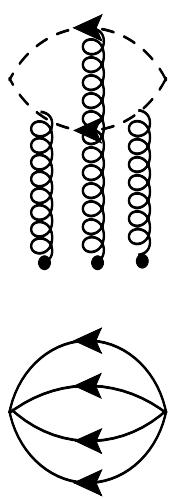

(3)

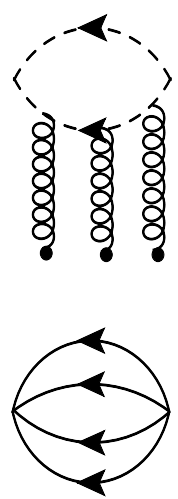

(4)<smiles>C1CCCCC1</smiles>

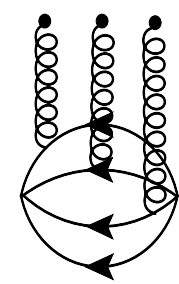

(5)

FIG. 3: Typical Feynman graphs for three-gluon condensates, truncation $k=\frac{3}{2}$.

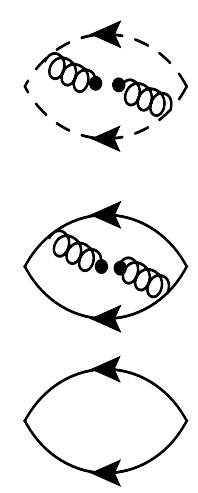

(1)

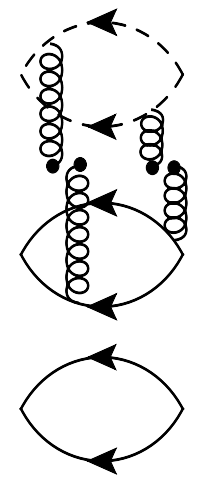

(2)

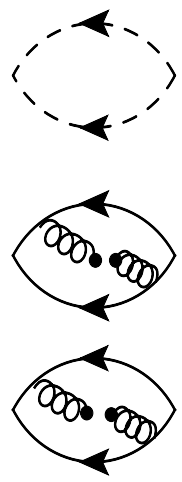

(3)

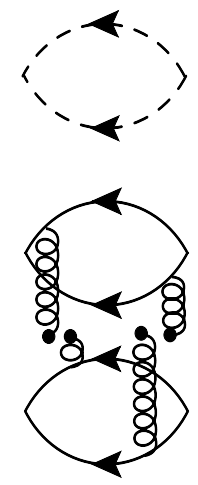

(4)

FIG. 4: Typical Feynman graphs for the $\left\langle\frac{\alpha_{s}}{\pi} G G\right\rangle^{2}$ condensates, truncation $k=2$.

After accomplishing the operator product expansion, we perform the Borel transform in regard to $P^{2}=-p^{2}$,

$$
\widehat{B}\left(P^{2}\right) \Pi(p)=\int_{\Delta^{2}}^{\infty} d s \rho_{Q C D}(s) \exp \left(-\frac{s}{T^{2}}\right),
$$

where the $\rho_{Q C D}(s)$ are the QCD spectral densities, the $T^{2}$ is the Borel parameter and $\widehat{B}\left(P^{2}\right)$ is the Borel operator which is defined as,

$$
\widehat{B}_{T^{2}}\left(P^{2}\right)=\lim _{\substack{-p^{2}, n \rightarrow \infty \\-p^{2} / n \rightarrow T^{2}}} \frac{\left(-p^{2}\right)^{(n+1)}}{n !}\left(\frac{d}{d p^{2}}\right)^{n} .
$$

Matching Eq.(8) with the spectral densities on the hadron side, we get the QCD sum rules,

$$
\lambda_{1,2,3,4}^{2} \exp \left(-\frac{M_{1,2,3,4}^{2}}{T^{2}}\right)=\int_{\Delta^{2}}^{s_{0}} d s \rho_{1,2,3,4}(s) \exp \left(-\frac{s}{T^{2}}\right),
$$




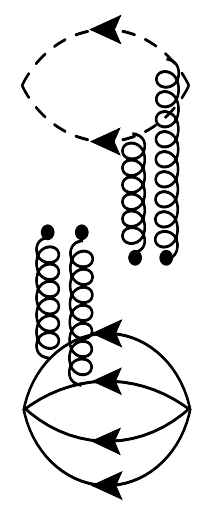

(5)

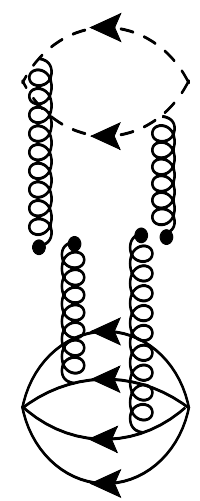

(6)<smiles>C1CCCCC1</smiles>

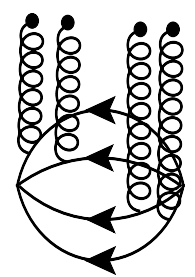

(7)

FIG. 5: Typical Feynman graphs for the $\left\langle\frac{\alpha_{s}}{\pi} G G\right\rangle^{2}$, truncation $k=2$.

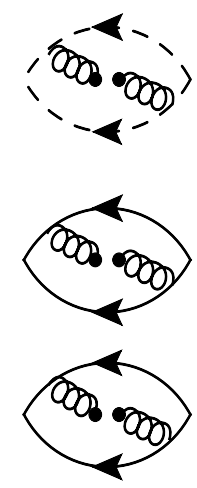

(1)

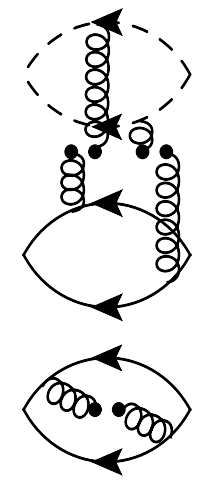

(2)

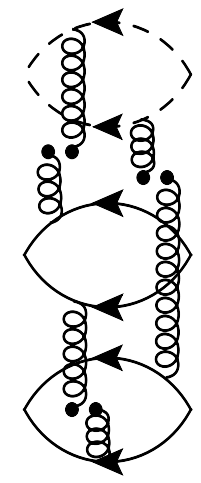

(3)

FIG. 6: Parts of typical Feynman graphs for the $\left\langle\frac{\alpha_{s}}{\pi} G G\right\rangle^{3}$ condensates with truncation $k=3$.

where the $\rho_{1,2,3,4}(s)$ are the QCD spectral densities for the currents $J_{1,2,3,4}$ and $\Delta^{2}=4 m_{c}^{2}$. An appendix is attached at the end of this paper, one can check the exact expressions of the spectral densities of the four currents there. As for the continuum threshold parameters $s_{0}$, we take experiential values,

$$
\sqrt{s_{0}}=M_{Z}+(0.5 \sim 0.7) \mathrm{GeV}
$$

where $M_{Z}$ are the masses of the ground states.

We differentiate the Eq.(10) with respect to $\tau=-\frac{1}{T^{2}}$ and eliminate the pole residues, the extracted masses can be written as,

$$
M_{1,2,3,4}^{2}=\frac{-\frac{d}{d \tau} \int_{\Delta^{2}}^{s_{0}} d s \rho_{1,2,3,4}(s) \exp (-s \tau)}{\int_{\Delta^{2}}^{s_{0}} d s \rho_{1,2,3,4}(s) \exp (-s \tau)} .
$$

\section{Numerical result and discussion}


We apply the standard values of the vacuum condensates $\langle\bar{q} q\rangle=-(0.24 \pm 0.01 \mathrm{GeV})^{3},\left\langle\bar{q} g_{s} \sigma G q\right\rangle=$ $m_{0}^{2}\langle\bar{q} q\rangle, m_{0}^{2}=(0.8 \pm 0.1) \mathrm{GeV}^{2},\left\langle\frac{\alpha_{s} G G}{\pi}\right\rangle=(0.33 \mathrm{GeV})^{4}$ at the energy scale $\mu=1 \mathrm{GeV}$ [27, 34], and choose the $\overline{M S}$ mass $m_{c}\left(m_{c}\right)=1.275 \pm 0.025 \mathrm{GeV}$ from the Particle Data Group [34]. Consider the energy-scale dependence for the above condensates [35],

$$
\begin{aligned}
\langle\bar{q} q\rangle(\mu) & =\langle\bar{q} q\rangle(1 \mathrm{GeV})\left[\frac{\alpha_{s}(1 \mathrm{GeV})}{\alpha_{s}(\mu)}\right]^{\frac{12}{33-2 n_{f}}}, \\
\left\langle\bar{q} g_{s} \sigma G q\right\rangle(\mu) & =\left\langle\bar{q} g_{s} \sigma G q\right\rangle(1 \mathrm{GeV})\left[\frac{\alpha_{s}(1 \mathrm{GeV})}{\alpha_{s}(\mu)}\right]^{\frac{2}{33-2 n_{f}}}, \\
m_{c}(\mu) & =m_{c}\left(m_{c}\right)\left[\frac{\alpha_{s}(\mu)}{\alpha_{s}\left(m_{c}\right)}\right]^{\frac{12}{33-2 n_{f}}}, \\
\alpha_{s}(\mu) & =\frac{1}{b_{0} t}\left[1-\frac{b_{1}}{b_{0}^{2}} \frac{\log t}{t}+\frac{b_{1}^{2}\left(\log ^{2} t-\log t-1\right)+b_{0} b_{2}}{b_{0}^{4} t^{2}}\right],
\end{aligned}
$$

where the related parameters are written as, where $t=\log \frac{\mu^{2}}{\Lambda^{2}}, b_{0}=\frac{33-2 n_{f}}{12 \pi}, b_{1}=\frac{153-19 n_{f}}{24 \pi^{2}}, b_{2}=$ $\frac{2857-\frac{5033}{9} n_{f}+\frac{325}{27} n_{f}^{2}}{128 \pi^{3}}, \Lambda=213 \mathrm{MeV}, 296 \mathrm{MeV}$ and $339 \mathrm{MeV}$ for the quark flavors $n_{f}=5,4$ and 3 , respectively [34]. In this paper, for the four constructed currents, we choose the flavor number $n_{f}=4$. We set $\alpha_{s}\langle\bar{\psi} \psi\rangle^{2}=-1.8 \times 10^{-4} \mathrm{GeV}^{6},\left\langle g_{s}^{3} f G G G\right\rangle=0.045 \mathrm{GeV}^{6}$ [26, 27, 36]. Compared with the heavy quarks, the masses of the light quarks $u$ and $d$ are too small to make much difference. So, we ignore the masses of the light quarks. The masses of baryon and anti-baryon are $M_{\Lambda_{c}}=M_{\bar{\Lambda}_{c}}=2.29 \mathrm{GeV}$ from the Particle Data Group 34]. Note that, Eq.(12) depends on the energy scale $\mu$ [29, 30], we apply the energy scale formula [28],

$$
\mu=\sqrt{M_{Z}^{2}-4 \mathbb{M}_{c}^{2}}
$$

to determine the best energy scales of the QCD spectral densities, where the $\mathbb{M}_{c}$ is the effective charmed quark mass, its updated value is $1.85 \mathrm{GeV}$ [37]. Combining above equations, we now find that the $M_{Z}$ depend on the threshold parameter $s_{0}$ and Borel parameter $T^{2}$. Of course, at first, we do not know the suitable energy scale, so we adjust the value of the $\mu$ via trial and error. From the energy scale formula Eq.(14), one can determine that the mass $M_{Z}$ increases with the parameter $\mu$, however, numerical results show that mass derived from Eq.(12) decreases with the increment of the parameter $\mu$, we can always find a best energy scale and determine the mass which satisfies the energy scale formula. As for the values of the $s_{0}$ and $T^{2}$, they are determined by the following two principles, firstly, the pole dominance criterion, here we define the pole contributions (PC) of the QCD sum rules as,

$$
\mathrm{PC}=\frac{\int_{4 m_{c}^{2}}^{s_{0}} d s \rho_{Q C D}(s) \exp \left(-\frac{s}{T^{2}}\right)}{\int_{4 m_{c}^{2}}^{\infty} d s \rho_{Q C D}(s) \exp \left(-\frac{s}{T^{2}}\right)},
$$

secondly, the convergence of the operator product expansion, for which, we define the contributions 
of the vacuum condensates of dimension $n$,

$$
D(n)=\frac{\int_{4 m_{c}^{2}}^{s_{0}} d s \rho_{Q C D ; n}(s) \exp \left(-\frac{s}{T^{2}}\right)}{\int_{4 m_{c}^{2}}^{s_{0}} d s \rho_{Q C D}(s) \exp \left(-\frac{s}{T^{2}}\right)} .
$$

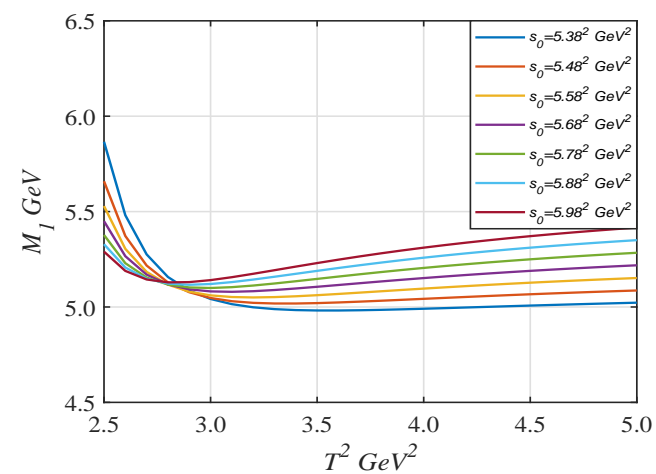

FIG. 7: The $M_{1}-T^{2}$ curves of the $J_{1}$ under different values of $\sqrt{s_{0}}$.

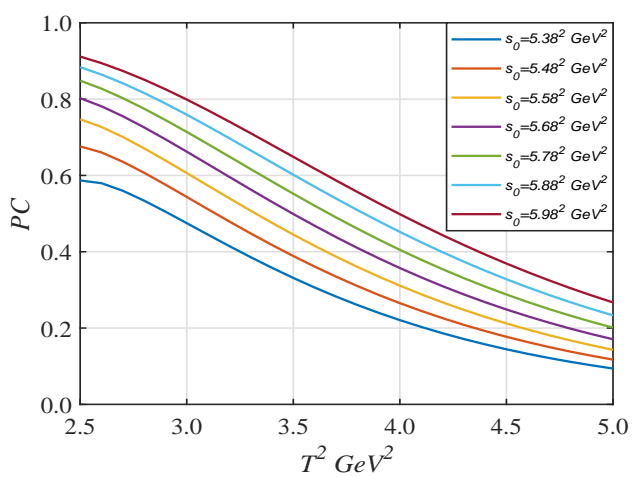

FIG. 8: The PC $-T^{2}$ curves of the $J_{1}$ under different values of $\sqrt{s_{0}}$.

Since the extracted mass from the QCD sum rules relies on the energy scale $\mu$ which differs with the threshold $s_{0}$, one should choose different suitable energy scale $\mu$ for each $s_{0}$, thus, we show the $M-T^{2}$ curves rather than $M-s_{0}$. We take the current $J_{1}$ as an example to show the detailed steps to determine the threshold $s_{0}$.

In Fig.7 and Fig.8, the numerical results of the $M_{1}-T^{2}$ and $\mathrm{PC}-T^{2}$ curves under different thresholds $\sqrt{s_{0}}$ are displayed. For $\sqrt{s_{0}}=5.78 \mathrm{GeV}$, according to the energy scale formula Eq.(14), the Borel window ranges from $3.9-4.3 \mathrm{GeV}^{2}$, the corresponding pole contribution is $43-35 \%$, which does not satisfy the pole dominance criterion. In the case of $\sqrt{s_{0}}=5.88 \mathrm{GeV}$ or $\sqrt{s_{0}}=5.98 \mathrm{GeV}$, the pole contribution has the smaller value compared with that of $\sqrt{s_{0}}=5.78 \mathrm{GeV}$. As for $\sqrt{s_{0}}=5.58 \mathrm{GeV}$, the mass should be $5.01 \mathrm{GeV}$ according to the energy scale formula Eq.(14), however, the minimum value of the $M-T^{2}$ curve is $5.05 \mathrm{GeV}$. The difference between the minimum value of the $M-T^{2}$ curve and mass satisfying Eq.(14) is even larger for either $\sqrt{s_{0}}=5.48 \mathrm{GeV}$ or $\sqrt{s_{0}}=5.38 \mathrm{GeV}$. Thus we have solid reason to set the threshold being $\sqrt{s_{0}}=5.68 \mathrm{GeV}$.

The $M_{1}-T^{2}$ graph of the current $J_{1}$ is shown in Fig.9, where the error bounds come from the uncertainties of the input parameters. A stable Borel platform is obtained with the parameter $T^{2}$ ranging from $3.4 \mathrm{GeV}^{2}$ to $3.8 \mathrm{GeV}^{2}$. The central value of the mass equals to $5.11 \mathrm{GeV}$ which satisfies the energy scale formula very well. As is discussed in Ref.[10], the contribution of the scattering states plays a tiny role. For a color-singlet-color-singlet type hexaquark bound state, naively we expect that its mass should be lower than the sum of the two baryon constituents, here, we find that the mass of the state is about $530 \mathrm{MeV}$ above that of the two $\Lambda_{c}$ baryons. All these facts show that the $\Lambda_{c} \Lambda_{c}$-type resonance with the $J^{P}=0^{+}$is a dibaryon state. The detailed numerical results are shown in Table I. 
TABLE I: The related numerical results extracted from the Borel windows of the four currents.

\begin{tabular}{cccccccc}
\hline \hline & $J^{P}$ & $\mu(\mathrm{GeV})$ & $T^{2}\left(\mathrm{GeV}^{2}\right)$ & $\sqrt{s_{0}}(\mathrm{GeV})$ & pole & $M(\mathrm{GeV})$ & $\lambda\left(10^{-4} \mathrm{GeV}^{8}\right)$ \\
\hline$J_{1}$ & $0^{+}$ & 3.52 & $3.4-3.8$ & $5.68 \pm 0.1$ & $(41-53) \%$ & $5.11_{-0.12}^{+0.15}$ & $17.55_{-2.68}^{+3.16}$ \\
$J_{2}$ & $0^{-}$ & 2.83 & $3.2-3.6$ & $5.28 \pm 0.1$ & $(42-55) \%$ & $4.66_{-0.06}^{+0.10}$ & $6.60_{-0.74}^{+0.17}$ \\
$J_{3}$ & $1^{+}$ & 3.35 & $3.4-3.8$ & $5.58 \pm 0.1$ & $(41-53) \%$ & $4.99_{-0.09}^{+0.10}$ & $6.16_{-0.97}^{+1.06}$ \\
$J_{4}$ & $1^{-}$ & 2.87 & $3.3-3.7$ & $5.38 \pm 0.1$ & $(42-56) \%$ & $4.68_{-0.08}^{+0.08}$ & $6.75_{-1.06}^{+1.12}$ \\
\hline \hline
\end{tabular}

TABLE II: Contributions of $D(k)$ for $\frac{3}{2} \leq k \leq 3$ in the Borel window of the $J_{1}$.

\begin{tabular}{ccccc}
\hline \hline & $k=\frac{3}{2}$ & $k=2$ & $k=\frac{5}{2}$ & $k=3$ \\
\hline$D(k)$ & $-3.07 \times 10^{-2}$ & $1.04 \times 10^{-2}$ & $-3.06 \times 10^{-5}$ & $-1.85 \times 10^{-5}$ \\
\hline \hline
\end{tabular}

Especially, in this paper, we consider the contributions of the truncation of the order $\mathcal{O}\left(\alpha_{s}^{k}\right)$ for $\frac{3}{2} \leq k \leq 3$ in detail. The vacuum condensates of the order $\mathcal{O}\left(\alpha_{s}^{\frac{3}{2}}\right)$ are $\left\langle\frac{\alpha_{s}}{\pi} G G\right\rangle\left\langle\bar{q} g_{s} \sigma G q\right\rangle,\left\langle g_{s}^{3} f G G G\right\rangle$ and $\left\langle\bar{q} g_{s} \sigma G q\right\rangle\left\langle\frac{\alpha_{s}}{\pi} G G\right\rangle\langle\bar{q} q\rangle$. For $k=2$, the related vacuum condensates are $\left\langle\frac{\alpha_{s}}{\pi} G G\right\rangle^{2},\left\langle\frac{\alpha_{s}}{\pi} G G\right\rangle^{2}\langle\bar{q} q\rangle^{2}$ and $\left\langle\frac{\alpha_{s}}{\pi} G G\right\rangle\left\langle\bar{q} g_{s} \sigma G q\right\rangle^{2}$. For $k=\frac{5}{2}$, the corresponding vacuum condensate is $\left\langle\frac{\alpha_{s}}{\pi} G G\right\rangle^{2}\left\langle\bar{q} g_{s} \sigma G q\right\rangle\langle\bar{q} q\rangle$. For $k=3$, the vacuum condensates in consideration are $\left\langle\frac{\alpha_{s}}{\pi} G G\right\rangle^{3}$ and $\left\langle g_{s}^{3} f G G G\right\rangle^{2}$. The contributions of the orders $\mathcal{O}\left(\alpha_{s}^{k}\right)$ are determined by the following equation,

$$
D(k)=\frac{\int_{4 m_{c}^{2}}^{s_{0}} d s \rho_{Q C D ; k}(s) \exp \left(-\frac{s}{T^{2}}\right)}{\int_{4 m_{c}^{2}}^{s_{0}} d s \rho_{Q C D}(s) \exp \left(-\frac{s}{T^{2}}\right)},
$$

where $k=\frac{3}{2}, 2, \frac{5}{2}, 3$ respectively. In Table II, all the condensate terms with $\frac{3}{2}<k \leq 3$ do play tiny roles, thus, it is reasonable for the QCD sum rules to choose the terms with $k \leq \frac{3}{2}$, they play the decisive roles. Furthermore, we calculate the contribution of the condensates related to the $\langle\bar{\psi} \psi\rangle^{2}$, similar to the $D(k)$, we define the $D(\langle\bar{\psi} \psi\rangle)$ as,

$$
D(\langle\bar{\psi} \psi\rangle)=\frac{\int_{4 m_{c}^{2}}^{s_{0}} d s \rho_{Q C D ;\langle\bar{\psi} \psi\rangle}(s) \exp \left(-\frac{s}{T^{2}}\right)}{\int_{4 m_{c}^{2}}^{s_{0}} d s \rho_{Q C D}(s) \exp \left(-\frac{s}{T^{2}}\right)}
$$

where the $\rho_{Q C D ;\langle\bar{\psi} \psi\rangle}$ refers to the spectral density contains the vacuum condensate $\langle\bar{\psi} \psi\rangle^{2}$. Its numerical value at the Borel window is $D(\langle\bar{\psi} \psi\rangle) \approx 8.7 \times 10^{-5}$, it shows that the vacuum condensate $\langle\bar{\psi} \psi\rangle^{2}$ does play a tiny role.

The numerical results of the masses for the currents $J_{2}, J_{3}$ and $J_{4}$ are shown in Fig.8 Fig.10 respectively. We also find Borel platforms for the $J_{2}, J_{3}$ and $J_{4}$. For the pseudoscalar current $J_{2}$, the central value of the mass in the platform is $4.66 \mathrm{GeV}$, just about $80 \mathrm{MeV}$ above the mass of the two baryon constituents. For the axialvector current $J_{3}$, the mass extracted from the Borel window is $4.99 \mathrm{GeV}$. As for the vector current $J_{4}$, the extracted mass is $4.68 \mathrm{GeV}$, which coincides very well with the center-of mass energy of the electron-positron annihilation with high integrated luminosity 


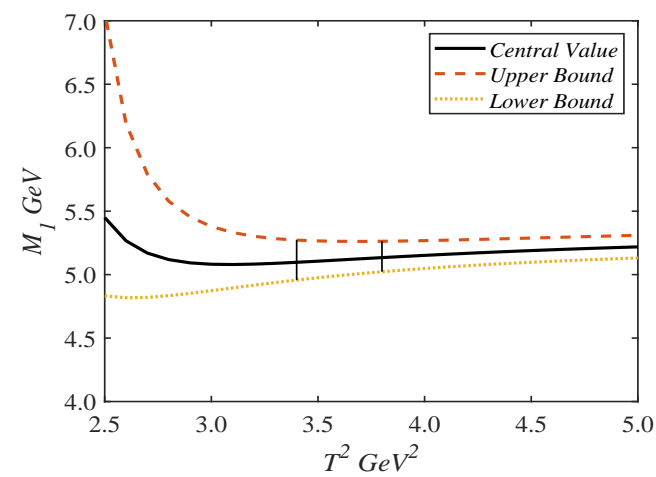

FIG. 9: The $M_{1}-T^{2}$ graph, dashed curve is drawn from the upper value of the input parameters and dotted curve is from the lower value.

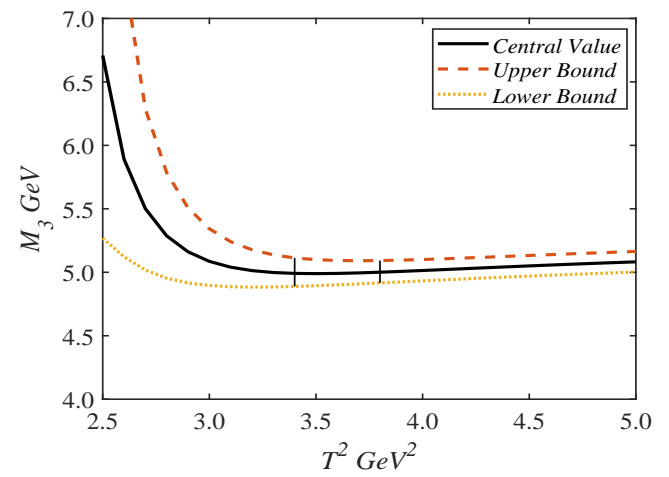

FIG. 11: The $M_{3}-T^{2}$ graph, dashed curve is drawn from the upper value of the input parameters and dotted curve is from the lower value.

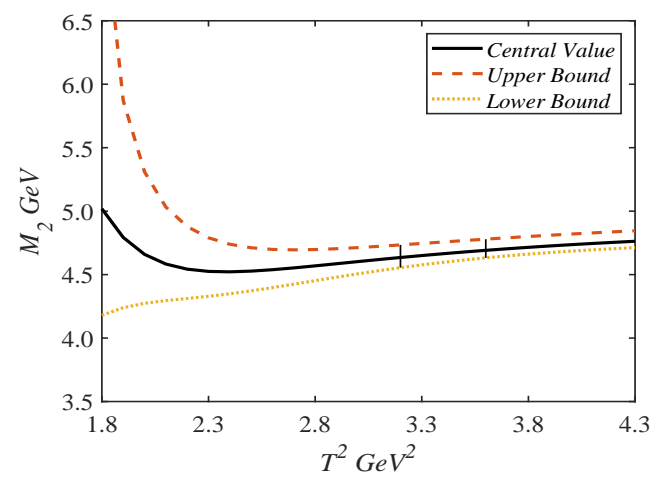

FIG. 10: The $M_{2}-T^{2}$ graph, dashed curve is drawn from the upper value of the input parameters and dotted curve is from the lower value.

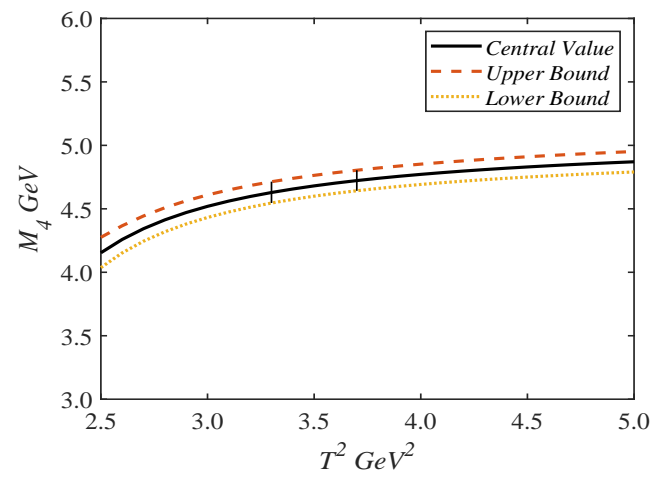

FIG. 12: The $M_{4}-T^{2}$ graph, dashed curve is drawn from the upper value of the input parameters and dotted curve is from the lower value.

7]. We are interested to work out whether or not there is a $Y$ state at around $4.68 \mathrm{GeV}$, thus it may be possible for us to find the relation between the $Y$ state and $\Lambda_{c} \bar{\Lambda}_{c}$ baryonium state with the $J^{P}=1^{-}$. The contributions $D(n)$ in the operator product expansion of each currents are displayed in Fig.13 Fig.16, as can be seen clearly on these graphs, for higher dimensions $(n \geq 10)$ in the regions of the Borel windows, they play the less important roles compared with the condensates with the low ones. The convergency of the operator product expansion holds well for the QCD sum rules. It is worth mentioning that it does not mean the consideration of the higher dimensions are meaningless. They maybe play important roles in stabilizing the Borel windows from which to extract the masses and pole residues and so on. As is shown in Fig.13 Fig.16, the vacuum condensates of dimension 6 (mainly $\left.\langle\bar{q} q\rangle^{2}\right)$ and dimension $8\left(\left\langle\bar{q} g_{s} \sigma G q\right\rangle\langle\bar{q} q\rangle\right)$ play the most important roles for these four states.

\section{Conclusion}




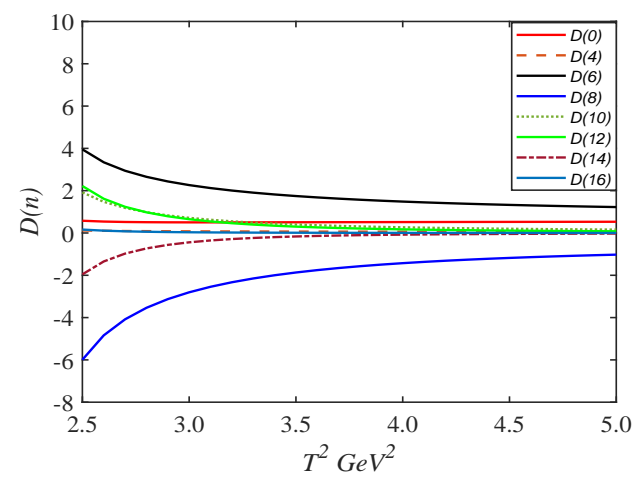

FIG. 13: The $D(n)-T^{2}$ graph for the $J_{1}$.

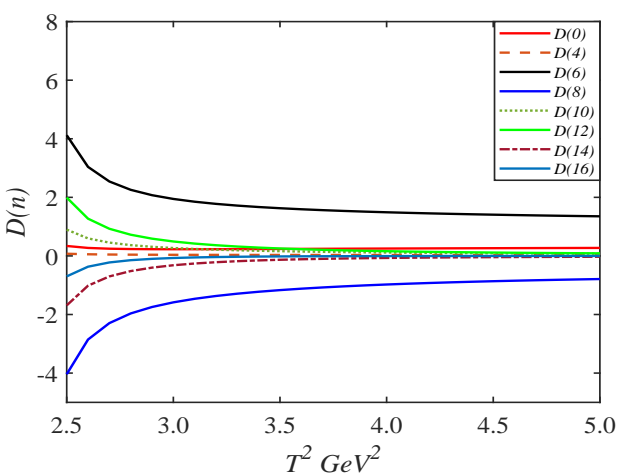

FIG. 15: The $D(n)-T^{2}$ graph for the $J_{3}$.

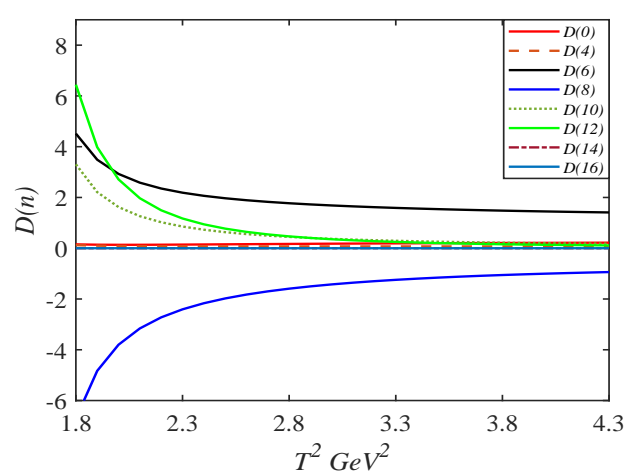

FIG. 14: The $D(n)-T^{2}$ graph for the $J_{2}$.

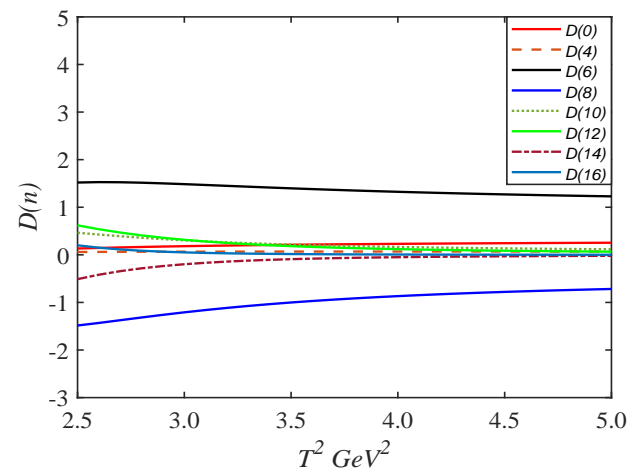

FIG. 16: The $D(n)-T^{2}$ graph for the $J_{4}$.

In this paper, we construct four currents $J_{1,2,3,4}$ with the $J^{P}=0^{+}, 0^{-}, 1^{+}, 1^{-}$respectively to study the $\Lambda_{c} \Lambda_{c}$ dibaryon and $\Lambda_{c} \bar{\Lambda}_{c}$ baryonium states. We acquire the Borel windows for these currents and extract the corresponding masses $5.11 \mathrm{GeV}, 4.66 \mathrm{GeV}, 4.99 \mathrm{GeV}$ and $4.68 \mathrm{GeV}$ respectively. They are all above the thresholds of the two baryon constituents, which is qualitatively in agreement with the results obtained in Ref.[11]. In contrary to the conclusion obtained in Ref.[11], we find the currents with the quantum numbers $J^{P}=0^{-}$and $1^{+}$also exist Borel platforms. The vacuum condensates are calculated up to dimension 16 , especially, for the current $J_{1}$, we consider all the vacuum condensates up to dimension 16 and choose the truncation of the operator product expansion of the order $\mathcal{O}\left(\alpha_{s}^{k}\right)$ with $k \leq 3$. The numerical results show that those vacuum condensates with $\frac{3}{2}<k \leq 3$ do play tiny roles and it is reasonable to discard these terms in the QCD sum rules. For the vector current $J_{4}$, the predicted mass $4.68 \mathrm{GeV}$ coincides very well with the center-of mass energy of the electron-positron annihilation with high integrated luminosity [7], we can confronted the prediction to the experimental 
data in the BESIII in the future.

[1] S.K. Choi et al. (Belle Collaboration), Phys. Rev. Lett. 91, (2003) 262001.

[2] B. Aubert et al. (BABAR Collaboration), Phys. Rev. D 77, (2008) 111101.

[3] F.K. Guo, C. Hanhart, U.G. Meissner, Q. Wang, Q. Zhao and B.S. Zou, Rev. Mod. Phys. 90, (2018) 015004.

[4] C.F. Qiao, Phys. Lett. B 639, (2006) 263.

[5] C.F. Qiao, J. Phys. G 35, (2008) 075008.

[6] Z.G. Wang and S.L Wan, J.Phys. G 34, (2007) 505-511.

[7] M. Ablikim, et al. arXiv:2011.07855 1 [hep-ph].

[8] N. Kodama, M. Oka and T. Hatsuda, Nucl. Phys. A 580, (1994) 445.

[9] H.X. Chen, E.L. Cui, W. Chen, T.G. Steele, and Sh.L. Zhu, Phys. Rev. C 91, (2015) 025204.

[10] Z.G. Wang, Eur. Phys. J. C 77, (2017) 642.

[11] B.D. Wan, L. Tang and C.F. Qiao, Eur. Phys. J. C80, (2020) 121.

[12] X.K. Dong, F.K. Guo and B.S. Zhou, arXiv:2101.01021 [hep-ph].

[13] S.S. Agaev, K. Azizi and H. Sundu, Phys. Rev. D 96, (2017) 034026.

[14] W. Chen and S.L. Zhu, Phys. Rev. D 81, (2010) 105018.

[15] W. Chen and S.L. Zhu, Phys. Rev. D 83, (2011) 034010.

[16] U. Ozdem and K. Azizi, Phys. Rev. D 97, (2018) 014010.

[17] S. H. Lee, M. Nielsen and U. Wiedner, J. Korean Phys. Soc. 55, (2009) 424.

[18] J.R. Zhang and M. Q. Huang, Commun. Theor. Phys. 54, (2010) 1075.

[19] J.R. Zhang, M. Zhong and M. Q. Huang, Phys. Lett. B 704, (2011) 312.

[20] R.D. Matheus, F. S. Navarra, M. Nielsen and C. M. Zanetti, Phys. Rev. D 80, (2009) 056002.

[21] S.H. Lee, A. Mihara, F.S. Navarra and M. Nielsen, Phys. Lett. B 661, (2008) 28.

[22] R.M. Albuquerque and M. Nielsen, Nucl.Phys. A 815, (2009) 53; Erratum, Nucl. Phys.A 857, (2011) 48.

[23] Z.Y. Di, Z.G. Wang and G.L. Yu, Commun. Theor. Phys. 71, (2019) 685.

[24] H.J. Lee, K.S. Kim and H. Kim, Phys. Rev. D 100, (2019) 034021.

[25] Y.J. Shi, Y. Xing and Z.X. Zhao, Eur. Phys. J. C 81, (2021) 156.

[26] M.A. Shifman, A.I. Vainshtein and V.I. Zakharov, Nucl. Phys. B 147, (1979) 385; Nucl. Phys. B147, (1979) 448.

[27] L.J. Reinders, H. Rubinstein and S. Yazaki, Phys. Rept. 127, (1985) 1.

[28] Z.G. Wang and T. Huang, Phys. Rev. D 89, (2014) 054019.

[29] Z.G. Wang and T. Huang, Eur. Phys. J. C 74, (2014) 2891.

[30] Z.G. Wang, Eur. Phys. J. C 76, (2016) 387.

[31] Z.G. Wang, Eur. Phys. J. C 76, (2016) 70.

[32] Z.G. Wang and T. huang, Eur. Phys. J. C 76, (2016) 43.

[33] P. Pascual and R. Tarrach, "QCD: Renormalization for the practitioner", Springer Berlin Heidelberg, (1984). 
[34] P.A. Zyla et al, Prog. Theor. Exp. Phys., (2020) 083 C01.

[35] S. Narison and R. Tarrach, Phys. Lett. 125 B, (1983) 217.

[36] P. Colangelo and A. Khodjamirian, arXiv:hep-ph/0010175.

[37] Z.G. Wang, Chin. Phys. C 41, (2017) 083103. 


\section{Appendix}

The right side of Eq.(10) can be expressed in the form $\mathbf{S}$,

$$
\mathbf{S}=\sum_{A, B, C, D, n, i} \mathbf{S}_{A, B, C, D}(n)_{i}
$$

where $A, B, C$ and $D$ refer to four types of integrals, $n$ are the dimension of the vacuum condensates. For the following expressions, $\tilde{m}^{2}=\frac{m_{c}^{2}}{(1-y) y}$ and $\bar{m}^{2}=\frac{(y+z) m_{c}^{2}}{y z}, y$ and $z$ are parameters introduced during the calculations of the integrals in the momentum space of the two heavy quarks, For type $A$ and $B, y_{i}=\frac{1}{2}\left(1-\sqrt{1-4 m_{c}^{2} / s}\right), y_{f}=\frac{1}{2}\left(1+\sqrt{1-4 m_{c}^{2} / s}\right)$ and $z_{i}=\frac{y m_{c}^{2}}{y s-m_{c}^{2}}$. For type $C$ and $D$, $y_{i}=0, y_{f}=1$ and $z_{i}=0$.

Type A for $J_{1}$

$$
\begin{aligned}
\mathbf{S}_{A}(12)_{1} & =\langle\bar{q} q\rangle^{4} \int_{4 m_{c}^{2}}^{s_{0}} d s \int_{y_{i}}^{y_{f}} d y e^{-\frac{s}{T^{2}}}\{y(y-1)\}\left\{\frac{-7\left(s-\tilde{m}^{2}\right)}{216 \pi^{2}}-\frac{7 s}{432 \pi^{2}}\right\} \\
& +m_{c}^{2}\langle\bar{q} q\rangle^{4} \int_{4 m_{c}^{2}}^{s_{0}} d s \int_{y_{i}}^{y_{f}} d y e^{-\frac{s}{T^{2}}}\left\{\frac{7}{432 \pi^{2}}\right\} \\
& +m_{c}^{2}\langle\bar{q} q\rangle^{4} \int_{4 m_{c}^{2}}^{s_{0}} d s \int_{y_{i}}^{y_{f}} d y e^{-\frac{s}{T^{2}}}\left\{\frac{7}{839808 \pi^{6}}\right\} \\
& +\langle\bar{q} q\rangle^{4} \int_{4 m_{c}^{2}}^{s_{0}} d s \int_{y_{i}}^{y_{f}} d y e^{-\frac{s}{T^{2}}}\{(y-1) y\}\left\{\frac{7\left(\tilde{m}^{2}-s\right)}{419904 \pi^{6}}-\frac{7 s}{839808 \pi^{6}}\right\} \\
& +m_{c}^{2}\langle\bar{q} q\rangle^{4} \int_{4 m_{c}^{2}}^{s_{0}} d s \int_{y_{i}}^{y_{f}} d y e^{-\frac{s}{T^{2}}}\left\{\frac{5}{11664 \pi^{4}}\right\} \\
& +\langle\bar{q} q\rangle^{4} \int_{4 m_{c}^{2}}^{s_{0}} d s \int_{y_{i}}^{y_{f}} d y e^{-\frac{s}{T^{2}}}\{(y-1) y\}\left\{\frac{5\left(\tilde{m}^{2}-s\right)}{5832 \pi^{4}}-\frac{5 s}{11664 \pi^{4}}\right\},
\end{aligned}
$$

$$
\begin{aligned}
\mathbf{S}_{A}(12)_{2} & =\langle\bar{q} q\rangle\left\langle g_{s}^{2} G G\right\rangle m_{c}^{2}\left\langle\bar{q} g_{s} \sigma G q\right\rangle \int_{4 m_{c}^{2}}^{s_{0}} d s \int_{y_{i}}^{y_{f}} d y e^{-\frac{s}{T^{2}}}\left\{-\frac{5}{110592 \pi^{6}}\right\} \\
& +\langle\bar{q} q\rangle\left\langle g_{s}^{2} G G\right\rangle\left\langle\bar{q} g_{s} \sigma G q\right\rangle \int_{4 m_{c}^{2}}^{s_{0}} d s \int_{y_{i}}^{y_{f}} d y e^{-\frac{s}{T^{2}}}\{(y-1) y\}\left\{\frac{5 s}{110592 \pi^{6}}-\frac{5\left(\tilde{m}^{2}-s\right)}{55296 \pi^{6}}\right\} \\
& +\left\langle g_{s}^{2} G G\right\rangle m_{c}^{2}\langle\bar{q} q\rangle\left\langle\bar{q} g_{s} \sigma G q\right\rangle \int_{4 m_{c}^{2}}^{s_{0}} d s \int_{y_{i}}^{y_{f}} d y e^{-\frac{s}{T^{2}}}\left\{-\frac{19}{147456 \pi^{6}}\right\} \\
& +\left\langle g_{s}^{2} G G\right\rangle\langle\bar{q} q\rangle\left\langle\bar{q} g_{s} \sigma G q\right\rangle \int_{4 m_{c}^{2}}^{s_{0}} d s \int_{y_{i}}^{y_{f}} d y e^{-\frac{s}{T^{2}}}\{(y-1) y\}\left\{\frac{19 s}{49152 \pi^{6}}-\frac{19 \tilde{m}^{2}}{73728 \pi^{6}}\right\}
\end{aligned}
$$




$$
\begin{aligned}
\mathbf{S}_{A}(14) & =\left\langle\bar{q} g_{s} \sigma G q\right\rangle\langle\bar{q} q\rangle^{3} \int_{4 m_{c}^{2}}^{s_{0}} d s \int_{y_{i}}^{y_{f}} d y e^{-\frac{s}{T^{2}}}\{(y-1) y\}\left\{\frac{7}{72 \pi^{2}}\right\} \\
& +\left\langle g_{s}^{2} G G\right\rangle\left\langle\bar{q} g_{s} \sigma G q\right\rangle^{2} \int_{4 m_{c}^{2}}^{s_{0}} d s \int_{y_{i}}^{y_{f}} d y e^{-\frac{s}{T^{2}}}\{(y-1) y\}\left\{-\frac{19}{196608 \pi^{6}}\right\} \\
& +\langle\bar{q} q\rangle^{2}\left\langle g_{s}^{2} G G\right\rangle^{2} \int_{4 m_{c}^{2}}^{s_{0}} d s \int_{y_{i}}^{y_{f}} d y e^{-\frac{s}{T^{2}}}\{(y-1) y\}\left\{-\frac{5}{1327104 \pi^{6}}\right\} \\
& +\langle\bar{q} q\rangle^{3}\left\langle\bar{q} g_{s} \sigma G q\right\rangle \int_{4 m_{c}^{2}}^{s_{0}} d s \int_{y_{i}}^{y_{f}} d y e^{-\frac{s}{T^{2}}}\{(y-1) y\}\left\{\frac{5}{3888 \pi^{4}}\right\}
\end{aligned}
$$

Type B for $J_{1}$

$$
\begin{aligned}
\mathbf{S}_{B}(0)= & \int_{4 m_{c}^{2}}^{s_{0}} d s \int_{y_{i}}^{y_{f}} d y \int_{z_{i}}^{1-y} d z e^{-\frac{s}{T^{2}}}\left\{-y z(y+z-1)^{5}\right\}\left\{\frac{\left(s-\bar{m}^{2}\right)^{7}}{58982400 \pi^{10}}+\frac{7 s\left(s-\bar{m}^{2}\right)^{6}}{117964800 \pi^{10}}\right\} \\
& +m_{c}^{2} \int_{4 m_{c}^{2}}^{s_{0}} d s \int_{y_{i}}^{y_{f}} d y \int_{z_{i}}^{1-y} d z e^{-\frac{s}{T^{2}}}\left\{-(y+z-1)^{5}\right\}\left\{\frac{7\left(s-\bar{m}^{2}\right)^{6}}{117964800 \pi^{10}}\right\}
\end{aligned}
$$

$$
\begin{aligned}
\mathbf{S}_{B}(4) & =2 m_{c}^{2}\left\langle g_{s}^{2} G G\right\rangle \int_{4 m_{c}^{2}}^{s_{0}} d s \int_{y_{i}}^{y_{f}} d y \int_{z_{i}}^{1-y} d z e^{-\frac{s}{T^{2}}}\left\{\frac{(y+z-1)^{5}}{y^{2}}\right\}\left\{\frac{7 y\left(s-\bar{m}^{2}\right)^{4}}{141557760 \pi^{10}}+\frac{7 s y\left(s-\bar{m}^{2}\right)^{3}}{70778880 \pi^{10}}\right\} \\
& +2 m_{c}^{2}\left\langle g_{s}^{2} G G\right\rangle \int_{4 m_{c}^{2}}^{s_{0}} d s \int_{y_{i}}^{y_{f}} d y \int_{z_{i}}^{1-y} d z e^{-\frac{s}{T^{2}}}\left\{\frac{(y+z-1)^{5}}{y^{2}}\right\}\left\{-\frac{7\left(s-\bar{m}^{2}\right)^{4}}{141557760 \pi^{10}}\right\} \\
& +2 m_{c}^{2}\left\langle g_{s}^{2} G G\right\rangle \int_{4 m_{c}^{2}}^{s_{0}} d s \int_{y_{i}}^{y_{f}} d y \int_{z_{i}}^{1-y} d z e^{-\frac{s}{T^{2}}}\left\{\frac{-z(y+z-1)^{5}}{y^{2}}\right\}\left\{-\frac{7\left(s-\bar{m}^{2}\right)^{4}}{141557760 \pi^{10}}-\frac{7 s\left(s-\bar{m}^{2}\right)^{3}}{70778880 \pi^{10}}\right\} \\
& +m_{c}^{2}\left\langle g_{s}^{2} G G\right\rangle \int_{4 m_{c}^{2}}^{s_{0}} d s \int_{y_{i}}^{y_{f}} d y \int_{z_{i}}^{1-y} d z e^{-\frac{s}{T^{2}}}\left\{-(y+z-1)^{3}\right\}\left\{\frac{19\left(s-\bar{m}^{2}\right)^{4}}{18874368 \pi^{10}}\right\} \\
& +\left\langle g_{s}^{2} G G\right\rangle \int_{4 m_{c}^{2}}^{s_{0}} d s \int_{y_{i}}^{y_{f}} d y \int_{z_{i}}^{1-y} d z e^{-\frac{s}{T^{2}}}\left\{-y z(y+z-1)^{3}\right\}\left\{\frac{19\left(s-\bar{m}^{2}\right)^{5}}{47185920 \pi^{10}}+\frac{19 s\left(s-\bar{m}^{2}\right)^{4}}{18874368 \pi^{10}}\right\} \\
& +m_{c}^{2}\left\langle g_{s}^{2} G G\right\rangle \int_{4 m_{c}^{2}}^{s_{0}} d s \int_{y_{i}}^{y_{f}} d y \int_{z_{i}}^{1-y} d z e^{-\frac{s}{T^{2}}}\left\{\frac{-(y+z-1)^{5}}{y z}\right\}\left\{-\frac{7\left(s-\bar{m}^{2}\right)^{4}}{188743680 \pi^{10}}\right\} \\
& +\left\langle g_{s}^{2} G G\right\rangle \int_{4 m_{c}^{2}}^{s_{0}} d s \int_{y_{i}}^{y_{f}} d y \int_{z_{i}}^{1-y} d z e^{-\frac{s}{T^{2}}}\left\{(y+z-1)^{5}\right\}\left\{\frac{7\left(s-\bar{m}^{2}\right)^{5}}{943718400 \pi^{10}}+\frac{7 s\left(s-\bar{m}^{2}\right)^{4}}{377487360 \pi^{10}}\right\}
\end{aligned}
$$




$$
\begin{aligned}
& \mathbf{S}_{B}(6)_{1}=\langle\bar{q} q\rangle^{2} \int_{4 m_{c}^{2}}^{s_{0}} d s \int_{y_{i}}^{y_{f}} d y \int_{z_{i}}^{1-y} d z e^{-\frac{s}{T^{2}}}\left\{y z(y+z-1)^{2}\right\}\left\{\frac{5\left(s-\bar{m}^{2}\right)^{4}}{9216 \pi^{6}}+\frac{5 s\left(s-\bar{m}^{2}\right)^{3}}{4608 \pi^{6}}\right\} \\
& +m_{c}^{2}\langle\bar{q} q\rangle^{2} \int_{4 m_{c}^{2}}^{s_{0}} d s \int_{y_{i}}^{y_{f}} d y \int_{z_{i}}^{1-y} d z e^{-\frac{s}{T^{2}}}\left\{(y+z-1)^{2}\right\}\left\{\frac{5\left(s-\bar{m}^{2}\right)^{3}}{4608 \pi^{6}}\right\} \\
& +m_{c}^{2} 2\langle\bar{q} q\rangle^{2} \int_{4 m_{c}^{2}}^{s_{0}} d s \int_{y_{i}}^{y_{f}} d y \int_{z_{i}}^{1-y} d z e^{-\frac{s}{T^{2}}}\left\{\frac{(y+z-1)^{4}}{y}\right\}\left\{-\frac{35\left(s-\bar{m}^{2}\right)^{3}}{7962624 \pi^{8}}-\frac{35 s\left(s-\bar{m}^{2}\right)^{2}}{5308416 \pi^{8}}\right\} \\
& +2 m_{c}^{2}\langle\bar{q} q\rangle^{2} \int_{4 m_{c}^{2}}^{s_{0}} d s \int_{y_{i}}^{y_{f}} d y \int_{z_{i}}^{1-y} d z e^{-\frac{s}{T^{2}}}\left\{\frac{z(y+z-1)^{4}}{y^{2}}\right\}\left\{-\frac{35\left(s-\bar{m}^{2}\right)^{3}}{7962624 \pi^{8}}-\frac{35 s\left(s-\bar{m}^{2}\right)^{2}}{5308416 \pi^{8}}\right\} \\
& +2\langle\bar{q} q\rangle^{2} \int_{4 m_{c}^{2}}^{s_{0}} d s \int_{y_{i}}^{y_{f}} d y \int_{z_{i}}^{1-y} d z e^{-\frac{s}{T^{2}}}\left\{\frac{z(y+z-1)^{4}}{y}\right\}\left\{\frac{35 s^{2} y\left(s-\bar{m}^{2}\right)^{2}}{7962624 \pi^{8}}+\frac{35 y\left(s-\bar{m}^{2}\right)^{4}}{15925248 \pi^{8}}\right\} \\
& +2\langle\bar{q} q\rangle^{2} \int_{4 m_{c}^{2}}^{s_{0}} d s \int_{y_{i}}^{y_{f}} d y \int_{z_{i}}^{1-y} d z e^{-\frac{s}{T^{2}}}\left\{\frac{z(y+z-1)^{4}}{y}\right\}\left\{\frac{35 s y\left(s-\bar{m}^{2}\right)^{3}}{3981312 \pi^{8}}-\frac{35\left(s-\bar{m}^{2}\right)^{4}}{23887872 \pi^{8}}\right\} \\
& +2\langle\bar{q} q\rangle^{2} \int_{4 m_{c}^{2}}^{s_{0}} d s \int_{y_{i}}^{y_{f}} d y \int_{z_{i}}^{1-y} d z e^{-\frac{s}{T^{2}}}\left\{\frac{z(y+z-1)^{4}}{y}\right\}\left\{-\frac{35 s\left(s-\bar{m}^{2}\right)^{3}}{11943936 \pi^{8}}\right\} \\
& +2\langle\bar{q} q\rangle^{2} \int_{4 m_{c}^{2}}^{s_{0}} d s \int_{y_{i}}^{y_{f}} d y \int_{z_{i}}^{1-y} d z e^{-\frac{s}{T^{2}}}\left\{\frac{z(y+z-1)^{4}}{y}\right\}\left\{-\frac{35 s^{2} y\left(s-\bar{m}^{2}\right)^{2}}{7962624 \pi^{8}}-\frac{35 y\left(s-\bar{m}^{2}\right)^{4}}{15925248 \pi^{8}}\right\} \\
& +2\langle\bar{q} q\rangle^{2} \int_{4 m_{c}^{2}}^{s_{0}} d s \int_{y_{i}}^{y_{f}} d y \int_{z_{i}}^{1-y} d z e^{-\frac{s}{T^{2}}}\left\{\frac{z(y+z-1)^{4}}{y}\right\}\left\{-\frac{35 s y\left(s-\bar{m}^{2}\right)^{3}}{3981312 \pi^{8}}+\frac{35\left(s-\bar{m}^{2}\right)^{4}}{95551488 \pi^{8}}\right\} \\
& +2\langle\bar{q} q\rangle^{2} \int_{4 m_{c}^{2}}^{s_{0}} d s \int_{y_{i}}^{y_{f}} d y \int_{z_{i}}^{1-y} d z e^{-\frac{s}{T^{2}}}\left\{\frac{z(y+z-1)^{4}}{y}\right\}\left\{\frac{35 s\left(s-\bar{m}^{2}\right)^{3}}{47775744 \pi^{8}}\right\} \\
& +m_{c}^{2}\langle\bar{q} q\rangle^{2} \int_{4 m_{c}^{2}}^{s_{0}} d s \int_{y_{i}}^{y_{f}} d y \int_{z_{i}}^{1-y} d z e^{-\frac{s}{T^{2}}}\left\{(y+z-1)^{2}\right\}\left\{\frac{7\left(s-\bar{m}^{2}\right)^{3}}{248832 \pi^{8}}\right\} \\
& +\langle\bar{q} q\rangle^{2} \int_{4 m_{c}^{2}}^{s_{0}} d s \int_{y_{i}}^{y_{f}} d y \int_{z_{i}}^{1-y} d z e^{-\frac{s}{T^{2}}}\left\{y z(y+z-1)^{2}\right\}\left\{\frac{7\left(s-\bar{m}^{2}\right)^{4}}{497664 \pi^{8}}+\frac{7 s\left(s-\bar{m}^{2}\right)^{3}}{248832 \pi^{8}}\right\},
\end{aligned}
$$




$$
\begin{aligned}
& \mathbf{S}_{B}(6)_{2}=m_{c}^{4}\langle\bar{\psi} \psi\rangle^{2} \int_{4 m_{c}^{2}}^{s_{0}} d s \int_{y_{i}}^{y_{f}} d y \int_{z_{i}}^{1-y} d z e^{-\frac{s}{T^{2}}}\left\{-\frac{(y+z-1)^{5}}{y^{2} z^{2}}\right\}\left\{-\frac{7\left(s-\bar{m}^{2}\right)^{2}}{159252480 \pi^{10}}\right\} \\
& +m_{c}^{2}\langle\bar{\psi} \psi\rangle^{2} \int_{4 m_{c}^{2}}^{s_{0}} d s \int_{y_{i}}^{y_{f}} d y \int_{z_{i}}^{1-y} d z e^{-\frac{s}{T^{2}}}\left\{\frac{(y+z-1)^{5}}{y z^{2}}\right\}\left\{-\frac{7(y-1)\left(s-\bar{m}^{2}\right)^{3}}{238878720 \pi^{10}}-\frac{7 s y\left(s-\bar{m}^{2}\right)^{2}}{159252480 \pi^{10}}\right\} \\
& +\langle\bar{\psi} \psi\rangle^{2} \int_{4 m_{c}^{2}}^{s_{0}} d s \int_{y_{i}}^{y_{f}} d y \int_{z_{i}}^{1-y} d z e^{-\frac{s}{T^{2}}}\left\{\frac{(y+z-1)^{6}}{(y-1) y z}\right\}\left\{\frac{7 s y\left(s-\bar{m}^{2}\right)^{3}}{716636160 \pi^{10}}-\frac{7(1-y)\left(s-\bar{m}^{2}\right)^{4}}{1433272320 \pi^{10}}\right\} \\
& +\langle\bar{\psi} \psi\rangle^{2} \int_{4 m_{c}^{2}}^{s_{0}} d s \int_{y_{i}}^{y_{f}} d y \int_{z_{i}}^{1-y} d z e^{-\frac{s}{T^{2}}}\left\{\frac{(y+z-1)^{5}}{(y-1) y}\right\}\left\{-\frac{7 s\left(s-\bar{m}^{2}\right)^{3}}{716636160 \pi^{10}}\right\} \\
& +\langle\bar{\psi} \psi\rangle^{2} \int_{4 m_{c}^{2}}^{s_{0}} d s \int_{y_{i}}^{y_{f}} d y \int_{z_{i}}^{1-y} d z e^{-\frac{s}{T^{2}}}\left\{(y+z-1)^{5}\right\}\left\{-\frac{7 s^{2}\left(s-\bar{m}^{2}\right)^{2}}{477757440 \pi^{10}}-\frac{7\left(s-\bar{m}^{2}\right)^{4}}{955514880 \pi^{10}}\right\} \\
& +\langle\bar{\psi} \psi\rangle^{2} \int_{4 m_{c}^{2}}^{s_{0}} d s \int_{y_{i}}^{y_{f}} d y \int_{z_{i}}^{1-y} d z e^{-\frac{s}{T^{2}}}\left\{(y+z-1)^{5}\right\}\left\{-\frac{7 s\left(s-\bar{m}^{2}\right)^{3}}{238878720 \pi^{10}}\right\} \\
& +m_{c}^{2}\langle\bar{\psi} \psi\rangle^{2} \int_{4 m_{c}^{2}}^{s_{0}} d s \int_{y_{i}}^{y_{f}} d y \int_{z_{i}}^{1-y} d z e^{-\frac{s}{T^{2}}}\left\{-\frac{(y+z-1)^{5}}{y z}\right\}\left\{-\frac{7\left(s-\bar{m}^{2}\right)^{3}}{59719680 \pi^{10}}-\frac{7 s\left(s-\bar{m}^{2}\right)^{2}}{39813120 \pi^{10}}\right\} \\
& +\langle\bar{\psi} \psi\rangle^{2} \int_{4 m_{c}^{2}}^{s_{0}} d s \int_{y_{i}}^{y_{f}} d y \int_{z_{i}}^{1-y} d z e^{-\frac{s}{T^{2}}}\left\{\frac{(y+z-1)^{6}}{(y-1) y z}\right\}\left\{\frac{49(1-y)\left(s-\bar{m}^{2}\right)^{4}}{5733089280 \pi^{10}}-\frac{49 s y\left(s-\bar{m}^{2}\right)^{3}}{2866544640 \pi^{10}}\right\} \\
& +\langle\bar{\psi} \psi\rangle^{2} \int_{4 m_{c}^{2}}^{s_{0}} d s \int_{y_{i}}^{y_{f}} d y \int_{z_{i}}^{1-y} d z e^{-\frac{s}{T^{2}}}\left\{\frac{(y+z-1)^{5}}{(y-1) y}\right\}\left\{\frac{49 s\left(s-\bar{m}^{2}\right)^{3}}{2866544640 \pi^{10}}\right\} \\
& +\langle\bar{\psi} \psi\rangle^{2} \int_{4 m_{c}^{2}}^{s_{0}} d s \int_{y_{i}}^{y_{f}} d y \int_{z_{i}}^{1-y} d z e^{-\frac{s}{T^{2}}}\left\{(y+z-1)^{5}\right\}\left\{\frac{49 s^{2}\left(s-\bar{m}^{2}\right)^{2}}{477757440 \pi^{10}}+\frac{49\left(s-\bar{m}^{2}\right)^{4}}{955514880 \pi^{10}}\right\} \\
& +\langle\bar{\psi} \psi\rangle^{2} \int_{4 m_{c}^{2}}^{s_{0}} d s \int_{y_{i}}^{y_{f}} d y \int_{z_{i}}^{1-y} d z e^{-\frac{s}{T^{2}}}\left\{(y+z-1)^{5}\right\}\left\{\frac{49 s\left(s-\bar{m}^{2}\right)^{3}}{238878720 \pi^{10}}\right\},
\end{aligned}
$$




$$
\begin{aligned}
& \mathbf{S}_{B}(6)_{3}=m_{c}^{2}\left\langle g_{s}^{3} f G G G\right\rangle \int_{4 m_{c}^{2}}^{s_{0}} d s \int_{y_{i}}^{y_{f}} d y \int_{z_{i}}^{1-y} d z e^{-\frac{s}{T^{2}}}\left\{(y+z-1)^{2}\right\}\left\{-\frac{\left(s-\bar{m}^{2}\right)^{3}}{524288 \pi^{10}}\right\} \\
& +\left\langle g_{s}^{3} f G G G\right\rangle \int_{4 m_{c}^{2}}^{s_{0}} d s \int_{y_{i}}^{y_{f}} d y \int_{z_{i}}^{1-y} d z e^{-\frac{s}{T^{2}}}\left\{y z(y+z-1)^{2}\right\}\left\{-\frac{\left(s-\bar{m}^{2}\right)^{4}}{1048576 \pi^{10}}-\frac{s\left(s-\bar{m}^{2}\right)^{3}}{524288 \pi^{10}}\right\} \\
& +m_{c}^{4}\left\langle g_{s}^{3} f G G G\right\rangle \int_{4 m_{c}^{2}}^{s_{0}} d s \int_{y_{i}}^{y_{f}} d y \int_{z_{i}}^{1-y} d z e^{-\frac{s}{T^{2}}}\left\{-\frac{(y+z-1)^{5}}{y^{4}}\right\}\left\{\frac{7\left(s-\bar{m}^{2}\right)^{2}}{94371840 \pi^{10}}\right\} \\
& +m_{c}^{2}\left\langle g_{s}^{3} f G G G\right\rangle \int_{4 m_{c}^{2}}^{s_{0}} d s \int_{y_{i}}^{y_{f}} d y \int_{z_{i}}^{1-y} d z e^{-\frac{s}{T^{2}}}\left\{\frac{(y+z-1)^{5}}{y^{3}}\right\}\left\{\frac{7(1-y)\left(s-\bar{m}^{2}\right)^{3}}{47185920 \pi^{10}}-\frac{7 s y\left(s-\bar{m}^{2}\right)^{2}}{31457280 \pi^{10}}\right\} \\
& +m_{c}^{2}\left\langle g_{s}^{3} f G G G\right\rangle \int_{4 m_{c}^{2}}^{s_{0}} d s \int_{y_{i}}^{y_{f}} d y \int_{z_{i}}^{1-y} d z e^{-\frac{s}{T^{2}}}\left\{-\frac{z(y+z-1)^{5}}{y^{3}}\right\}\left\{\frac{49\left(s-\bar{m}^{2}\right)^{3}}{283115520 \pi^{10}}+\frac{49 s\left(s-\bar{m}^{2}\right)^{2}}{188743680 \pi^{10}}\right\} \\
& +\left\langle g_{s}^{3} f G G G\right\rangle \int_{4 m_{c}^{2}}^{s_{0}} d s \int_{y_{i}}^{y_{f}} d y \int_{z_{i}}^{1-y} d z e^{-\frac{s}{T^{2}}}\left\{-\frac{z(y+z-1)^{5}}{y^{2}}\right\}\left\{-\frac{7\left(s-\bar{m}^{2}\right)^{4}}{283115520 \pi^{10}}-\frac{7 s\left(s-\bar{m}^{2}\right)^{3}}{141557760 \pi^{10}}\right\} \\
& +m_{c}^{2}\left\langle g_{s}^{3} f G G G\right\rangle \int_{4 m_{c}^{2}}^{s_{0}} d s \int_{y_{i}}^{y_{f}} d y \int_{z_{i}}^{1-y} d z e^{-\frac{s}{T^{2}}}\left\{-\frac{z(y+z-1)^{5}}{y^{3}}\right\}\left\{\frac{7\left(s-\bar{m}^{2}\right)^{3}}{283115520 \pi^{10}}+\frac{7 s\left(s-\bar{m}^{2}\right)^{2}}{188743680 \pi^{10}}\right\} \\
& +m_{c}^{4}\left\langle g_{s}^{3} f G G G\right\rangle \int_{4 m_{c}^{2}}^{s_{0}} d s \int_{y_{i}}^{y_{f}} d y \int_{z_{i}}^{1-y} d z e^{-\frac{s}{T^{2}}}\left\{-\frac{(y+z-1)^{5}}{y^{3} z}\right\}\left\{-\frac{7\left(s-\bar{m}^{2}\right)^{2}}{94371840 \pi^{10}}\right\} \\
& +m_{c}^{2}\left\langle g_{s}^{3} f G G G\right\rangle \int_{4 m_{c}^{2}}^{s_{0}} d s \int_{y_{i}}^{y_{f}} d y \int_{z_{i}}^{1-y} d z e^{-\frac{s}{T^{2}}}\left\{-\frac{(y+z-1)^{5}}{y^{2} z}\right\}\left\{\frac{7 s y\left(s-\bar{m}^{2}\right)^{2}}{47185920 \pi^{10}}-\frac{7(1-y)\left(s-\bar{m}^{2}\right)^{3}}{70778880 \pi^{10}}\right\} \\
& +m_{c}^{2}\left\langle g_{s}^{3} f G G G\right\rangle \int_{4 m_{c}^{2}}^{s_{0}} d s \int_{y_{i}}^{y_{f}} d y \int_{z_{i}}^{1-y} d z e^{-\frac{s}{T^{2}}}\left\{\frac{(y+z-1)^{5}}{y^{2}}\right\}\left\{-\frac{7\left(s-\bar{m}^{2}\right)^{3}}{283115520 \pi^{10}}-\frac{7 s\left(s-\bar{m}^{2}\right)^{2}}{188743680 \pi^{10}}\right\} \\
& +\left\langle g_{s}^{3} f G G G\right\rangle \int_{4 m_{c}^{2}}^{s_{0}} d s \int_{y_{i}}^{y_{f}} d y \int_{z_{i}}^{1-y} d z e^{-\frac{s}{T^{2}}}\left\{\frac{(y+z-1)^{5}}{y}\right\}\left\{\frac{7\left(s-\bar{m}^{2}\right)^{4}}{377487360 \pi^{10}}+\frac{7 s\left(s-\bar{m}^{2}\right)^{3}}{188743680 \pi^{10}}\right\} \\
& +m_{c}^{2}\left\langle g_{s}^{3} f G G G\right\rangle \int_{4 m_{c}^{2}}^{s_{0}} d s \int_{y_{i}}^{y_{f}} d y \int_{z_{i}}^{1-y} d z e^{-\frac{s}{T^{2}}}\left\{\frac{(y+z-1)^{5}}{y^{2}}\right\}\left\{-\frac{7\left(s-\bar{m}^{2}\right)^{3}}{283115520 \pi^{10}}-\frac{7 s\left(s-\bar{m}^{2}\right)^{2}}{188743680 \pi^{10}}\right\},
\end{aligned}
$$

$$
\begin{aligned}
\mathbf{S}_{B}(8)_{1} & =\left\langle\bar{q} g_{s} \sigma G q\right\rangle\langle\bar{q} q\rangle \int_{4 m_{c}^{2}}^{s_{0}} d s \int_{y_{i}}^{y_{f}} d y \int_{z_{i}}^{1-y} d z e^{-\frac{s}{T^{2}}}\{-y z(y+z-1)\}\left\{-\frac{5\left(s-\bar{m}^{2}\right)^{3}}{2304 \pi^{6}}-\frac{5 s\left(s-\bar{m}^{2}\right)^{2}}{1536 \pi^{6}}\right\} \\
& +m_{c}^{2}\left\langle\bar{q} g_{s} \sigma G q\right\rangle\langle\bar{q} q\rangle \int_{4 m_{c}^{2}}^{s_{0}} d s \int_{y_{i}}^{y_{f}} d y \int_{z_{i}}^{1-y} d z e^{-\frac{s}{T^{2}}}\{-y-z+1\}\left\{-\frac{5\left(s-\bar{m}^{2}\right)^{2}}{1536 \pi^{6}}\right\} \\
& +\left\langle\bar{q} g_{s} \sigma G q\right\rangle m_{c}^{2}\langle\bar{q} q\rangle \int_{4 m_{c}^{2}}^{s_{0}} d s \int_{y_{i}}^{y_{f}} d y \int_{z_{i}}^{1-y} d z e^{-\frac{s}{T^{2}}}\{-y-z+1\}\left\{-\frac{13\left(s-\bar{m}^{2}\right)^{2}}{6144 \pi^{6}}\right\} \\
& +\left\langle\bar{q} g_{s} \sigma G q\right\rangle\langle\bar{q} q\rangle \int_{4 m_{c}^{2}}^{s_{0}} d s \int_{y_{i}}^{y_{f}} d y \int_{z_{i}}^{1-y} d z e^{-\frac{s}{T^{2}}}\{-y z(y+z-1)\}\left\{-\frac{13\left(s-\bar{m}^{2}\right)^{3}}{9216 \pi^{6}}-\frac{13 s\left(s-\bar{m}^{2}\right)^{2}}{6144 \pi^{6}}\right\},
\end{aligned}
$$




$$
\begin{aligned}
& \mathbf{S}_{B}(8)_{2}=m_{c}^{4}\left\langle g_{s}^{2} G G\right\rangle^{2} \int_{4 m_{c}^{2}}^{s_{0}} d s \int_{y_{i}}^{y_{f}} d y \int_{z_{i}}^{1-y} d z e^{-\frac{s}{T^{2}}}\left\{\frac{(y+z-1)^{5}}{y^{2} z^{2}}\right\}\left\{\frac{7\left(\bar{m}^{2}-s\right)}{424673280 \pi^{10}}-\frac{7 s}{849346560 \pi^{10}}\right\} \\
& +m_{c}^{2}\left\langle g_{s}^{2} G G\right\rangle^{2} \int_{4 m_{c}^{2}}^{s_{0}} d s \int_{y_{i}}^{y_{f}} d y \int_{z_{i}}^{1-y} d z e^{-\frac{s}{T^{2}}}\left\{-\frac{(y+z-1)^{6}}{(y-1) y^{2} z^{2}}\right\}\left\{\frac{7 s(1-y)\left(s-\bar{m}^{2}\right)^{2}}{424673280 \pi^{10}}-\frac{7 s y\left(s-\bar{m}^{2}\right)}{424673280 \pi^{10}}\right\} \\
& +m_{c}^{2}\left\langle g_{s}^{2} G G\right\rangle^{2} \int_{4 m_{c}^{2}}^{s_{0}} d s \int_{y_{i}}^{y_{f}} d y \int_{z_{i}}^{1-y} d z e^{-\frac{s}{T^{2}}}\left\{-\frac{(y+z-1)^{5}}{(y-1) y^{2} z}\right\}\left\{\frac{7 s\left(s-\bar{m}^{2}\right)}{424673280 \pi^{10}}\right\} \\
& +m_{c}^{2}\left\langle g_{s}^{2} G G\right\rangle^{2} \int_{4 m_{c}^{2}}^{s_{0}} d s \int_{y_{i}}^{y_{f}} d y \int_{z_{i}}^{1-y} d z e^{-\frac{s}{T^{2}}}\left\{-\frac{(y+z-1)^{5}}{y z}\right\}\left\{\frac{7 s\left(s-\bar{m}^{2}\right)}{141557760 \pi^{10}}+\frac{7\left(s-\bar{m}^{2}\right)^{2}}{283115520 \pi^{10}}\right\} \\
& +m_{c}^{2}\left\langle g_{s}^{2} G G\right\rangle^{2} \int_{4 m_{c}^{2}}^{s_{0}} d s \int_{y_{i}}^{y_{f}} d y \int_{z_{i}}^{1-y} d z e^{-\frac{s}{T^{2}}}\left\{-\frac{(y+z-1)^{5}}{y z}\right\}\left\{\frac{7 s^{2}}{849346560 \pi^{10}}\right\} \\
& +m_{c}^{2}\left\langle g_{s}^{2} G G\right\rangle^{2} \int_{4 m_{c}^{2}}^{s_{0}} d s \int_{y_{i}}^{y_{f}} d y \int_{z_{i}}^{1-y} d z e^{-\frac{s}{T^{2}}}\left\{-\frac{(y+z-1)^{3}}{y z}\right\}\left\{-\frac{145\left(s-\bar{m}^{2}\right)^{2}}{905969664 \pi^{10}}\right\} \\
& +\left\langle g_{s}^{2} G G\right\rangle^{2} \int_{4 m_{c}^{2}}^{s_{0}} d s \int_{y_{i}}^{y_{f}} d y \int_{z_{i}}^{1-y} d z e^{-\frac{s}{T^{2}}}\left\{(y+z-1)^{3}\right\}\left\{\frac{259\left(s-\bar{m}^{2}\right)^{3}}{4076863488 \pi^{10}}+\frac{259 s\left(s-\bar{m}^{2}\right)^{2}}{2717908992 \pi^{10}}\right\} \\
& +\left\langle g_{s}^{2} G G\right\rangle^{2} \int_{4 m_{c}^{2}}^{s_{0}} d s \int_{y_{i}}^{y_{f}} d y \int_{z_{i}}^{1-y} d z e^{-\frac{s}{T^{2}}}\left\{-(y+z-1)^{4}\right\}\left\{-\frac{83 s^{2}\left(s-\bar{m}^{2}\right)}{2717908992 \pi^{10}}-\frac{83\left(s-\bar{m}^{2}\right)^{3}}{2717908992 \pi^{10}}\right\} \\
& +\left\langle g_{s}^{2} G G\right\rangle^{2} \int_{4 m_{c}^{2}}^{s_{0}} d s \int_{y_{i}}^{y_{f}} d y \int_{z_{i}}^{1-y} d z e^{-\frac{s}{T^{2}}}\left\{-(y+z-1)^{4}\right\}\left\{-\frac{83 s\left(s-\bar{m}^{2}\right)^{2}}{905969664 \pi^{10}}\right\} \\
& +m_{c}^{2}\left\langle g_{s}^{2} G G\right\rangle^{2} \int_{4 m_{c}^{2}}^{s_{0}} d s \int_{y_{i}}^{y_{f}} d y \int_{z_{i}}^{1-y} d z e^{-\frac{s}{T^{2}}}\{-y-z+1\}\left\{\frac{199\left(s-\bar{m}^{2}\right)^{2}}{150994944 \pi^{10}}\right\} \\
& +\left\langle g_{s}^{2} G G\right\rangle^{2} \int_{4 m_{c}^{2}}^{s_{0}} d s \int_{y_{i}}^{y_{f}} d y \int_{z_{i}}^{1-y} d z e^{-\frac{s}{T^{2}}}\{-y z(y+z-1)\}\left\{\frac{199\left(s-\bar{m}^{2}\right)^{3}}{226492416 \pi^{10}}+\frac{199 s\left(s-\bar{m}^{2}\right)^{2}}{150994944 \pi^{10}}\right\},
\end{aligned}
$$




$$
\begin{aligned}
& \mathbf{S}_{B}(10)_{1}=\left\langle\bar{q} g_{s} \sigma G q\right\rangle^{2} \int_{4 m_{c}^{2}}^{s_{0}} d s \int_{y_{i}}^{y_{f}} d y \int_{z_{i}}^{1-y} d z e^{-\frac{s}{T^{2}}}\{y z\}\left\{\frac{5\left(s-\bar{m}^{2}\right)^{2}}{6144 \pi^{6}}+\frac{5 s\left(s-\bar{m}^{2}\right)}{6144 \pi^{6}}\right\} \\
& +m_{c}^{2}\left\langle\bar{q} g_{s} \sigma G q\right\rangle^{2} \int_{4 m_{c}^{2}}^{s_{0}} d s \int_{y_{i}}^{y_{f}} d y \int_{z_{i}}^{1-y} d z e^{-\frac{s}{T^{2}}}\left\{-\frac{5\left(\bar{m}^{2}-s\right)}{6144 \pi^{6}}\right\} \\
& +m_{c}^{2}\left\langle\bar{q} g_{s} \sigma G q\right\rangle^{2} \int_{4 m_{c}^{2}}^{s_{0}} d s \int_{y_{i}}^{y_{f}} d y \int_{z_{i}}^{1-y} d z e^{-\frac{s}{T^{2}}}\left\{\frac{-13\left(\bar{m}^{2}-s\right)}{12288 \pi^{6}}\right\} \\
& +\left\langle\bar{q} g_{s} \sigma G q\right\rangle^{2} \int_{4 m_{c}^{2}}^{s_{0}} d s \int_{y_{i}}^{y_{f}} d y \int_{z_{i}}^{1-y} d z e^{-\frac{s}{T^{2}}}\{y z\}\left\{\frac{13\left(s-\bar{m}^{2}\right)^{2}}{12288 \pi^{6}}+\frac{13 s\left(s-\bar{m}^{2}\right)}{12288 \pi^{6}}\right\} \\
& +m_{c}^{2}\left\langle\bar{q} g_{s} \sigma G q\right\rangle^{2} \int_{4 m_{c}^{2}}^{s_{0}} d s \int_{y_{i}}^{y_{f}} d y \int_{z_{i}}^{1-y} d z e^{-\frac{s}{T^{2}}}\left\{\frac{59\left(\bar{m}^{2}-s\right)}{1179648 \pi^{6}}\right\} \\
& +\left\langle\bar{q} g_{s} \sigma G q\right\rangle^{2} \int_{4 m_{c}^{2}}^{s_{0}} d s \int_{y_{i}}^{y_{f}} d y \int_{z_{i}}^{1-y} d z e^{-\frac{s}{T^{2}}}\{y z\}\left\{-\frac{59\left(s-\bar{m}^{2}\right)^{2}}{1179648 \pi^{6}}-\frac{59 s\left(s-\bar{m}^{2}\right)}{1179648 \pi^{6}}\right\} \\
& +m_{c}^{2}\left\langle\bar{q} g_{s} \sigma G q\right\rangle^{2} \int_{4 m_{c}^{2}}^{s_{0}} d s \int_{y_{i}}^{y_{f}} d y \int_{z_{i}}^{1-y} d z e^{-\frac{s}{T^{2}}}\left\{\frac{(y+z-1)^{2}}{y z}\right\}\left\{\frac{-\left(\bar{m}^{2}-s\right)}{24576 \pi^{6}}\right\} \\
& +\left\langle\bar{q} g_{s} \sigma G q\right\rangle^{2} \int_{4 m_{c}^{2}}^{s_{0}} d s \int_{y_{i}}^{y_{f}} d y \int_{z_{i}}^{1-y} d z e^{-\frac{s}{T^{2}}}\left\{-(y+z-1)^{2}\right\}\left\{-\frac{109\left(s-\bar{m}^{2}\right)}{7077888 \pi^{6}}-\frac{109 s\left(s-\bar{m}^{2}\right)}{7077888 \pi^{6}}\right\} \\
& +\left\langle\bar{q} g_{s} \sigma G q\right\rangle^{2} \int_{4 m_{c}^{2}}^{s_{0}} d s \int_{y_{i}}^{y_{f}} d y \int_{z_{i}}^{1-y} d z e^{-\frac{s}{T^{2}}}\left\{(y+z-1)^{3}\right\}\left\{-\frac{35 s\left(s-\bar{m}^{2}\right)}{884736 \pi^{6}}-\frac{35\left(s-\bar{m}^{2}\right)^{2}}{1769472 \pi^{6}}\right\} \\
& +\left\langle\bar{q} g_{s} \sigma G q\right\rangle^{2} \int_{4 m_{c}^{2}}^{s_{0}} d s \int_{y_{i}}^{y_{f}} d y \int_{z_{i}}^{1-y} d z e^{-\frac{s}{T^{2}}}\left\{(y+z-1)^{3}\right\}\left\{-\frac{35 s^{2}}{5308416 \pi^{6}}\right\},
\end{aligned}
$$




$$
\begin{aligned}
& \mathbf{S}_{B}(10)_{2}=2 m_{c}^{2}\langle\bar{q} q\rangle^{2}\left\langle g_{s}^{2} G G\right\rangle \int_{4 m_{c}^{2}}^{s_{0}} d s \int_{y_{i}}^{y_{f}} d y \int_{z_{i}}^{1-y} d z e^{-\frac{s}{T^{2}}}\left\{-\frac{(y+z-1)^{2}}{y^{2}}\right\}\left\{-\frac{5 y\left(\bar{m}^{2}-s\right)}{27648 \pi^{6}}+\frac{5\left(\bar{m}^{2}-s\right)}{27648 \pi^{6}}\right\} \\
& +2 m_{c}^{2}\langle\bar{q} q\rangle^{2}\left\langle g_{s}^{2} G G\right\rangle \int_{4 m_{c}^{2}}^{s_{0}} d s \int_{y_{i}}^{y_{f}} d y \int_{z_{i}}^{1-y} d z e^{-\frac{s}{T^{2}}}\left\{-\frac{(y+z-1)^{2}}{y^{2}}\right\}\left\{\frac{5 s y}{55296 \pi^{6}}\right\} \\
& +2 m_{c}^{2}\langle\bar{q} q\rangle^{2}\left\langle g_{s}^{2} G G\right\rangle \int_{4 m_{c}^{2}}^{s_{0}} d s \int_{y_{i}}^{y_{f}} d y \int_{z_{i}}^{1-y} d z e^{-\frac{s}{T^{2}}}\left\{\frac{z(y+z-1)^{2}}{y^{2}}\right\}\left\{\frac{5\left(\bar{m}^{2}-s\right)}{27648 \pi^{6}}-\frac{5 s}{55296 \pi^{6}}\right\} \\
& +m_{c}^{2}\left\langle g_{s}^{2} G G\right\rangle\langle\bar{q} q\rangle^{2} \int_{4 m_{c}^{2}}^{s_{0}} d s \int_{y_{i}}^{y_{f}} d y \int_{z_{i}}^{1-y} d z e^{-\frac{s}{T^{2}}}\left\{-\frac{19\left(\bar{m}^{2}-s\right)}{73728 \pi^{6}}\right\} \\
& +\left\langle g_{s}^{2} G G\right\rangle\langle\bar{q} q\rangle^{2} \int_{4 m_{c}^{2}}^{s_{0}} d s \int_{y_{i}}^{y_{f}} d y \int_{z_{i}}^{1-y} d z e^{-\frac{s}{T^{2}}}\{y z\}\left\{\frac{19\left(s-\bar{m}^{2}\right)^{2}}{73728 \pi^{6}}+\frac{19 s\left(s-\bar{m}^{2}\right)}{73728 \pi^{6}}\right\} \\
& +m_{c}^{2}\left\langle g_{s}^{2} G G\right\rangle\langle\bar{q} q\rangle^{2} \int_{4 m_{c}^{2}}^{s_{0}} d s \int_{y_{i}}^{y_{f}} d y \int_{z_{i}}^{1-y} d z e^{-\frac{s}{T^{2}}}\left\{\frac{(y+z-1)^{2}}{y z}\right\}\left\{\frac{5\left(\bar{m}^{2}-s\right)}{36864 \pi^{6}}\right\} \\
& +\left\langle g_{s}^{2} G G\right\rangle\langle\bar{q} q\rangle^{2} \int_{4 m_{c}^{2}}^{s_{0}} d s \int_{y_{i}}^{y_{f}} d y \int_{z_{i}}^{1-y} d z e^{-\frac{s}{T^{2}}}\left\{-(y+z-1)^{2}\right\}\left\{\frac{5\left(s-\bar{m}^{2}\right)^{2}}{73728 \pi^{6}}+\frac{5 s\left(s-\bar{m}^{2}\right)}{73728 \pi^{6}}\right\} \\
& +m_{c}^{2}\langle\bar{q} q\rangle^{2}\left\langle g_{s}^{2} G G\right\rangle \int_{4 m_{c}^{2}}^{s_{0}} d s \int_{y_{i}}^{y_{f}} d y \int_{z_{i}}^{1-y} d z e^{-\frac{s}{T^{2}}}\left\{-\frac{5\left(\bar{m}^{2}-s\right)}{27648 \pi^{6}}\right\} \\
& +\langle\bar{q} q\rangle^{2}\left\langle g_{s}^{2} G G\right\rangle \int_{4 m_{c}^{2}}^{s_{0}} d s \int_{y_{i}}^{y_{f}} d y \int_{z_{i}}^{1-y} d z e^{-\frac{s}{T^{2}}}\{y z\}\left\{\frac{5\left(s-\bar{m}^{2}\right)^{2}}{27648 \pi^{6}}+\frac{5 s\left(s-\bar{m}^{2}\right)}{27648 \pi^{6}}\right\} \\
& \left\langle g_{s}^{2} G G\right\rangle\langle\bar{q} q\rangle^{2} \int_{4 m_{c}^{2}}^{s_{0}} d s \int_{y_{i}}^{y_{f}} d y \int_{z_{i}}^{1-y} d z e^{-\frac{s}{T^{2}}}\left\{\frac{z(y+z-1)^{2}}{y^{2}}\right\}\left\{\frac{\bar{m}^{2}-s}{186624 \pi^{8}}-\frac{s}{373248 \pi^{8}}\right\} \\
& \left\langle g_{s}^{2} G G\right\rangle\langle\bar{q} q\rangle^{2} \int_{4 m_{c}^{2}}^{s_{0}} d s \int_{y_{i}}^{y_{f}} d y \int_{z_{i}}^{1-y} d z e^{-\frac{s}{T^{2}}}\left\{\frac{-(y+z-1)^{2}}{y^{2}}\right\}\left\{\frac{(1-y)\left(\bar{m}^{2}-s\right)}{186624 \pi^{8}}+\frac{s y}{373248 \pi^{8}}\right\},
\end{aligned}
$$

$$
\begin{aligned}
\mathbf{S}_{B}(12)_{1} \quad & =2 m_{c}^{2}\left\langle\bar{q} g_{s} \sigma G q\right\rangle\langle\bar{q} q\rangle\left\langle g_{s}^{2} G G\right\rangle \int_{4 m_{c}^{2}}^{s_{0}} d s \int_{y_{i}}^{y_{f}} d y \int_{z_{i}}^{1-y} d z e^{-\frac{s}{T^{2}}}\left\{\frac{y+z-1}{y^{2}}\right\}\left\{-\frac{5 y}{27648 \pi^{6}}+\frac{5}{27648 \pi^{6}}\right\} \\
& +2 m_{c}^{2}\left\langle\bar{q} g_{s} \sigma G q\right\rangle\langle\bar{q} q\rangle\left\langle g_{s}^{2} G G\right\rangle \int_{4 m_{c}^{2}}^{s_{0}} d s \int_{y_{i}}^{y_{f}} d y \int_{z_{i}}^{1-y} d z e^{-\frac{s}{T^{2}}}\left\{-\frac{z(y+z-1)}{y^{2}}\right\}\left\{\frac{5}{27648 \pi^{6}}\right\} \\
& +\left\langle g_{s}^{2} G G\right\rangle\left\langle\bar{q} g_{s} \sigma G q\right\rangle\langle\bar{q} q\rangle \int_{4 m_{c}^{2}}^{s_{0}} d s \int_{y_{i}}^{y_{f}} d y \int_{z_{i}}^{1-y} d z e^{-\frac{s}{T^{2}}}\{y+z-1\}\left\{\frac{5 \bar{m}^{2}}{36864 \pi^{6}}-\frac{5 s}{24576 \pi^{6}}\right\} \\
& +m_{c}^{2}\left\langle g_{s}^{2} G G\right\rangle\left\langle\bar{q} g_{s} \sigma G q\right\rangle\langle\bar{q} q\rangle \int_{4 m_{c}^{2}}^{s_{0}} d s \int_{y_{i}}^{y_{f}} d y \int_{z_{i}}^{1-y} d z e^{-\frac{s}{T^{2}}}\left\{-\frac{y+z-1}{y z}\right\}\left\{\frac{5}{36864 \pi^{6}}\right\},
\end{aligned}
$$




$$
\begin{aligned}
\mathbf{S}_{B}(12)_{2} & =2 m_{c}^{2}\langle\bar{q} q\rangle^{4} \int_{4 m_{c}^{2}}^{s_{0}} d s \int_{y_{i}}^{y_{f}} d y \int_{z_{i}}^{1-y} d z e^{-\frac{s}{T^{2}}}\left\{\frac{y+z-1}{y}\right\}\left\{\frac{25}{31104 \pi^{4}}\right\} \\
& +2 m_{c}^{2}\langle\bar{q} q\rangle^{4} \int_{4 m_{c}^{2}}^{s_{0}} d s \int_{y_{i}}^{y_{f}} d y \int_{z_{i}}^{1-y} d z e^{-\frac{s}{T^{2}}}\left\{-\frac{z(z+y-1)}{y^{2}}\right\}\left\{-\frac{25}{31104 \pi^{4}}\right\} \\
& +2\langle\bar{q} q\rangle^{4} \int_{4 m_{c}^{2}}^{s_{0}} d s \int_{y_{i}}^{y_{f}} d y \int_{z_{i}}^{1-y} d z e^{-\frac{s}{T^{2}}}\left\{-\frac{z(y+z-1)}{y}\right\}\left\{-\frac{25 y\left(\bar{m}^{2}-s\right)}{15552 \pi^{4}}+\frac{25\left(\bar{m}^{2}-s\right)}{23328 \pi^{4}}\right\} \\
& +2\langle\bar{q} q\rangle^{4} \int_{4 m_{c}^{2}}^{s_{0}} d s \int_{y_{i}}^{y_{f}} d y \int_{z_{i}}^{1-y} d z e^{-\frac{s}{T^{2}}}\left\{-\frac{z(y+z-1)}{y}\right\}\left\{\frac{25 s y}{15552 \pi^{4}}-\frac{25 s}{46656 \pi^{4}}\right\} \\
& +2\langle\bar{q} q\rangle^{4} \int_{4 m_{c}^{2}}^{s_{0}} d s \int_{y_{i}}^{y_{f}} d y \int_{z_{i}}^{1-y} d z e^{-\frac{s}{T^{2}}}\left\{-\frac{z(y+z-1)}{y}\right\}\left\{\frac{25 y\left(\bar{m}^{2}-s\right)}{15552 \pi^{4}}-\frac{25\left(\bar{m}^{2}-s\right)}{93312 \pi^{4}}\right\} \\
& +2\langle\bar{q} q\rangle^{4} \int_{4 m_{c}^{2}}^{s_{0}} d s \int_{y_{i}}^{y_{f}} d y \int_{z_{i}}^{1-y} d z e^{-\frac{s}{T^{2}}}\left\{-\frac{z(y+z-1)}{y}\right\}\left\{-\frac{25 s y}{15552 \pi^{4}}+\frac{25 s}{186624 \pi^{4}}\right\}
\end{aligned}
$$

$$
\begin{aligned}
\mathbf{S}_{B}(12)_{3} & \left.=\langle\bar{\psi} \psi\rangle^{2}\langle\bar{q} q\rangle^{2} \int_{4 m_{c}^{2}}^{s_{0}} d s \int_{y_{i}}^{y_{f}} d y \int_{z_{i}}^{1-y} d z e^{-\frac{s}{T^{2}}}\left\{-\frac{(y+z-1)^{3}}{(y-1) y z}\right)\right\}\left\{\frac{35(y-1)\left(\bar{m}^{2}-s\right)}{1119744 \pi^{6}}-\frac{35 s y}{2239488 \pi^{6}}\right\} \\
& +\langle\bar{\psi} \psi\rangle^{2}\langle\bar{q} q\rangle^{2} \int_{4 m_{c}^{2}}^{s_{0}} d s \int_{y_{i}}^{y_{f}} d y \int_{z_{i}}^{1-y} d z e^{-\frac{s}{T^{2}}}\left\{-\frac{(y+z-1)^{2}}{(y-1) y}\right\}\left\{\frac{35 s}{2239488 \pi^{6}}\right\} \\
& +\langle\bar{\psi} \psi\rangle^{2}\langle\bar{q} q\rangle^{2} \int_{4 m_{c}^{2}}^{s_{0}} d s \int_{y_{i}}^{y_{f}} d y \int_{z_{i}}^{1-y} d z e^{-\frac{s}{T^{2}}}\left\{-(y+z-1)^{2}\right\}\left\{-\frac{35\left(\bar{m}^{2}-s\right)}{186624 \pi^{6}}+\frac{35 s}{186624 \pi^{6}}\right\} \\
& +m_{c}^{2}\langle\bar{\psi} \psi\rangle^{2}\langle\bar{q} q\rangle^{2} \int_{4 m_{c}^{2}}^{s_{0}} d s \int_{y_{i}}^{y_{f}} d y \int_{z_{i}}^{1-y} d z e^{-\frac{s}{T^{2}}}\left\{-\frac{(y+z-1)^{2}}{y z^{2}}\right\}\left\{\frac{5(1-y)}{186624 \pi^{6}}\right\} \\
& +\langle\bar{\psi} \psi\rangle^{2}\langle\bar{q} q\rangle^{2} \int_{4 m_{c}^{2}}^{s_{0}} d s \int_{y_{i}}^{y_{f}} d y \int_{z_{i}}^{1-y} d z e^{-\frac{s}{T^{2}}}\left\{-\frac{(y+z-1)^{3}}{(y-1) y z}\right\}\left\{\frac{5(1-y)\left(\bar{m}^{2}-s\right)}{279936 \pi^{6}}+\frac{5 s y}{559872 \pi^{6}}\right\} \\
& +\langle\bar{\psi} \psi\rangle^{2}\langle\bar{q} q\rangle^{2} \int_{4 m_{c}^{2}}^{s_{0}} d s \int_{y_{i}}^{y_{f}} d y \int_{z_{i}}^{1-y} d z e^{-\frac{s}{T^{2}}}\left\{-\frac{(y+z-1)^{2}}{(y-1) y}\right\}\left\{-\frac{5 s}{559872 \pi^{6}}\right\} \\
& +\langle\bar{\psi} \psi\rangle^{2}\langle\bar{q} q\rangle^{2} \int_{4 m_{c}^{2}}^{s_{0}} d s \int_{y_{i}}^{y_{f}} d y \int_{z_{i}}^{1-y} d z e^{-\frac{s}{T^{2}}}\left\{-(y+z-1)^{2}\right\}\left\{\frac{5\left(\bar{m}^{2}-s\right)}{186624 \pi^{6}}-\frac{5 s}{186624 \pi^{6}}\right\} \\
& +m_{c}^{2}\langle\bar{\psi} \psi\rangle^{2}\langle\bar{q} q\rangle^{2} \int_{4 m_{c}^{2}}^{s_{0}} d s \int_{y_{i}}^{y_{f}} d y \int_{z_{i}}^{1-y} d z e^{-\frac{s}{T^{2}}}\left\{\frac{(y+z-1)^{2}}{y z}\right\}\left\{-\frac{5}{46656 \pi^{6}}\right\},
\end{aligned}
$$

$$
\begin{aligned}
\mathbf{S}_{B}(12)_{4} & =m_{c}^{2}\left\langle g_{s}^{2} G G\right\rangle^{3} \int_{4 m_{c}^{2}}^{s_{0}} d s \int_{y_{i}}^{y_{f}} d y \int_{z_{i}}^{1-y} d z e^{-\frac{s}{T^{2}}}\left\{-\frac{y+z-1}{y z}\right\}\left\{-\frac{3143}{65229815808 \pi^{10}}\right\} \\
& +\left\langle g_{s}^{2} G G\right\rangle^{3} \int_{4 m_{c}^{2}}^{s_{0}} d s \int_{y_{i}}^{y_{f}} d y \int_{z_{i}}^{1-y} d z e^{-\frac{s}{T^{2}}}\{y+z-1\}\left\{\frac{2383 s}{97844723712 \pi^{10}}-\frac{2383\left(\bar{m}^{2}-s\right)}{48922361856 \pi^{10}}\right\} \\
& +\left\langle g_{s}^{2} G G\right\rangle^{3} \int_{4 m_{c}^{2}}^{s_{0}} d s \int_{y_{i}}^{y_{f}} d y \int_{z_{i}}^{1-y} d z e^{-\frac{s}{T^{2}}}\left\{-(y+z-1)^{2}\right\}\left\{\frac{103\left(\bar{m}^{2}-s\right)}{32614907904 \pi^{10}}-\frac{103 s}{32614907904 \pi^{10}}\right\}
\end{aligned}
$$




$$
\begin{aligned}
\mathbf{S}_{B}(12)_{5} & =m_{c}^{2}\left\langle g_{s}^{3} f G G G\right\rangle^{2} \int_{4 m_{c}^{2}}^{s_{0}} d s \int_{y_{i}}^{y_{f}} d y \int_{z_{i}}^{1-y} d z e^{-\frac{s}{T^{2}}}\left\{-\frac{y+z-1}{y z}\right\}\left\{-\frac{1}{50331648 \pi^{10}}\right\} \\
& +\left\langle g_{s}^{3} f G G G\right\rangle^{2} \int_{4 m_{c}^{2}}^{s_{0}} d s \int_{y_{i}}^{y_{f}} d y \int_{z_{i}}^{1-y} d z e^{-\frac{s}{T^{2}}}\{y+z-1\}\left\{\frac{s}{150994944 \pi^{10}}-\frac{\bar{m}^{2}-s}{75497472 \pi^{10}}\right\} \\
& +\left\langle g_{s}^{3} f G G G\right\rangle^{2} \int_{4 m_{c}^{2}}^{s_{0}} d s \int_{y_{i}}^{y_{f}} d y \int_{z_{i}}^{1-y} d z e^{-\frac{s}{T^{2}}}\left\{-(y+z-1)^{2}\right\}\left\{-\frac{\bar{m}^{2}-s}{25165824 \pi^{10}}+\frac{s}{25165824 \pi^{10}}\right\},
\end{aligned}
$$

$$
\begin{aligned}
\mathbf{S}_{B}(14)_{1} & =\left\langle g_{s}^{2} G G\right\rangle^{2}\langle\bar{q} q\rangle^{2} \int_{4 m_{c}^{2}}^{s_{0}} d s \int_{y_{i}}^{y_{f}} d y \int_{z_{i}}^{1-y} d z e^{-\frac{s}{T^{2}}}\left\{\frac{259}{63700992 \pi^{6}}\right\} \\
& +\left\langle g_{s}^{2} G G\right\rangle^{2}\langle\bar{q} q\rangle^{2} \int_{4 m_{c}^{2}}^{s_{0}} d s \int_{y_{i}}^{y_{f}} d y \int_{z_{i}}^{1-y} d z e^{-\frac{s}{T^{2}}}\{y+z-1\}\left\{-\frac{83}{10616832 \pi^{6}}\right\}
\end{aligned}
$$

$$
\mathbf{S}_{B}(14)_{2}=\left\langle g_{s}^{2} G G\right\rangle\left\langle\bar{q} g_{s} \sigma G q\right\rangle^{2} \int_{4 m_{c}^{2}}^{s_{0}} d s \int_{y_{i}}^{y_{f}} d y \int_{z_{i}}^{1-y} d z e^{-\frac{s}{T^{2}}}\left\{-\frac{5}{294912 \pi^{6}}\right\}
$$

$$
\begin{aligned}
\mathbf{S}_{B}(14)_{3} & =2\left\langle\bar{q} g_{s} \sigma G q\right\rangle\langle\bar{q} q\rangle^{3} \int_{4 m_{c}^{2}}^{s_{0}} d s \int_{y_{i}}^{y_{f}} d y \int_{z_{i}}^{1-y} d z e^{-\frac{s}{T^{2}}}\left\{\frac{z}{y}\right\}\left\{-\frac{25 y}{31104 \pi^{4}}+\frac{25}{46656 \pi^{4}}\right\} \\
& +2\left\langle\bar{q} g_{s} \sigma G q\right\rangle\langle\bar{q} q\rangle^{3} \int_{4 m_{c}^{2}}^{s_{0}} d s \int_{y_{i}}^{y_{f}} d y \int_{z_{i}}^{1-y} d z e^{-\frac{s}{T^{2}}}\left\{\frac{z}{y}\right\}\left\{\frac{25 y}{31104 \pi^{4}}-\frac{25}{186624 \pi^{4}}\right\}
\end{aligned}
$$

$$
\begin{aligned}
\mathbf{S}_{B}(14)_{4} & =\langle\bar{q} q\rangle\left\langle\bar{q} g_{s} \sigma G q\right\rangle\langle\bar{\psi} \psi\rangle^{2} \int_{4 m_{c}^{2}}^{s_{0}} d s \int_{y_{i}}^{y_{f}} d y \int_{z_{i}}^{1-y} d z e^{-\frac{s}{T^{2}}}\left\{\frac{(y+z-1)^{2}}{(y-1) y z}\right\}\left\{-\frac{5 y}{279936 \pi^{6}}+\frac{5}{279936 \pi^{6}}\right\} \\
& +\langle\bar{q} q\rangle\left\langle\bar{q} g_{s} \sigma G q\right\rangle\langle\bar{\psi} \psi\rangle^{2} \int_{4 m_{c}^{2}}^{s_{0}} d s \int_{y_{i}}^{y_{f}} d y \int_{z_{i}}^{1-y} d z e^{-\frac{s}{T^{2}}}\{y+z-1\}\left\{\frac{5}{186624 \pi^{6}}\right\} \\
& +\langle\bar{q} q\rangle\left\langle\bar{q} g_{s} \sigma G q\right\rangle\langle\bar{\psi} \psi\rangle^{2} \int_{4 m_{c}^{2}}^{s_{0}} d s \int_{y_{i}}^{y_{f}} d y \int_{z_{i}}^{1-y} d z e^{-\frac{s}{T^{2}}}\left\{\frac{(y+z-1)^{2}}{(y-1) y z}\right\}\left\{\frac{35 y}{1119744 \pi^{6}}-\frac{35}{1119744 \pi^{6}}\right\} \\
& +\langle\bar{q} q\rangle\left\langle\bar{q} g_{s} \sigma G q\right\rangle\langle\bar{\psi} \psi\rangle^{2} \int_{4 m_{c}^{2}}^{s_{0}} d s \int_{y_{i}}^{y_{f}} d y \int_{z_{i}}^{1-y} d z e^{-\frac{s}{T^{2}}}\{y+z-1\}\left\{-\frac{35}{186624 \pi^{6}}\right\} .
\end{aligned}
$$

Type C for $J_{1}$ 


$$
\begin{gathered}
\mathbf{S}_{C}(14)_{1} \quad=\left\langle\bar{q} g_{s} \sigma G q\right\rangle\langle\bar{q} q\rangle^{3} \int_{y_{i}}^{y_{f}} d y e^{-\frac{\tilde{m}^{2}}{T^{2}}}\{(y-1) y\}\left\{\frac{7 \tilde{m}^{2}}{108 \pi^{2}}+\frac{7 \tilde{m}^{4}}{432 \pi^{2} T^{2}}\right\} \\
+m_{c}^{2}\left\langle\bar{q} g_{s} \sigma G q\right\rangle\langle\bar{q} q\rangle^{3} \int_{y_{i}}^{y_{f}} d y e^{-\frac{\tilde{m}^{2}}{T^{2}}}\left\{-\frac{7}{432 \pi^{2}}-\frac{7 \tilde{m}^{2}}{432 \pi^{2} T^{2}}\right\} \\
+m_{c}^{2}\langle\bar{q} q\rangle^{3}\left\langle\bar{q} g_{s} \sigma G q\right\rangle \int_{y_{i}}^{y_{f}} d y e^{-\frac{\tilde{m}^{2}}{T^{2}}}\left\{-\frac{5}{23328 \pi^{4}}-\frac{5 \tilde{m}^{2}}{23328 \pi^{4} T^{2}}\right\} \\
+\langle\bar{q} q\rangle^{3}\left\langle\bar{q} g_{s} \sigma G q\right\rangle \int_{y_{i}}^{y_{f}} d y e^{-\frac{\tilde{m}^{2}}{T^{2}}}\{(y-1) y\}\left\{\frac{5 \tilde{m}^{2}}{5832 \pi^{4}}+\frac{5 \tilde{m}^{4}}{23328 \pi^{4} T^{2}}\right\} \\
\mathbf{S}_{C}(14)_{2}=m_{c}^{2}\left\langle g_{s}^{2} G G\right\rangle\left\langle\bar{q} g_{s} \sigma G q\right\rangle^{2} \int_{y_{i}}^{y_{f}} d y e^{-\frac{\tilde{m}^{2}}{T^{2}}}\left\{\frac{19}{1179648 \pi^{6}}+\frac{19 \tilde{m}^{2}}{1179648 \pi^{6} T^{2}}\right\} \\
+\left\langle g_{s}^{2} G G\right\rangle\left\langle\bar{q} g_{s} \sigma G q\right\rangle^{2} \int_{y_{i}}^{y_{f}} d y e^{-\frac{\tilde{m}^{2}}{T^{2}}}\{(y-1) y\}\left\{-\frac{19 \tilde{m}^{2}}{294912 \pi^{6}}-\frac{19 \tilde{m}^{4}}{1179648 \pi^{6} T^{2}}\right\} \\
\quad \mathbf{S}_{C}(14)_{3} \quad=m_{c}^{2}\langle\bar{q} q\rangle^{2}\left\langle g_{s}^{2} G G\right\rangle^{2} \int_{y_{i}}^{y_{f}} d y e^{-\frac{\tilde{m}^{2}}{T^{2}}}\left\{\frac{5}{7962624 \pi^{6}}+\frac{5 \tilde{m}^{2}}{7962624 \pi^{6} T^{2}}\right\} \\
+\langle\bar{q} q\rangle^{2}\left\langle g_{s}^{2} G G\right\rangle^{2} \int_{y_{i}}^{y_{f}} d y e^{-\frac{\tilde{m}^{2}}{T^{2}}}\{(y-1) y\}\left\{-\frac{5 \tilde{m}^{2}}{1990656 \pi^{6}}-\frac{5 \tilde{m}^{4}}{7962624 \pi^{6} T^{2}}\right\}
\end{gathered}
$$




$$
\begin{aligned}
& \mathbf{S}_{C}(16)_{1}=\frac{1}{6}\left\langle\bar{q} g_{s} \sigma G q\right\rangle^{2}\langle\bar{q} q\rangle^{2} \int_{y_{i}}^{y_{f}} d y e^{-\frac{\tilde{m}^{2}}{T^{2}}}\{(y-1) y\}\left\{-\frac{7}{32 \pi^{2}}+\frac{17 \tilde{m}^{6}}{192 \pi^{2} T^{6}}-\frac{7 \tilde{m}^{4}}{64 \pi^{2} T^{4}}-\frac{7 \tilde{m}^{2}}{32 \pi^{2} T^{2}}\right\} \\
& +\frac{1}{6}\left\langle\bar{q} g_{s} \sigma G q\right\rangle^{2}\langle\bar{q} q\rangle^{2} m_{c}^{2} \int_{y_{i}}^{y_{f}} d y e^{-\frac{\tilde{m}^{2}}{T^{2}}}\left\{\frac{7 \tilde{m}^{4}}{192 \pi^{2} T^{6}}\right\} \\
& +\langle\bar{q} q\rangle^{2}\left\langle\bar{q} g_{s} \sigma G q\right\rangle^{2} m_{c}^{2} \int_{y_{i}}^{y_{f}} d y e^{-\frac{\tilde{m}^{2}}{T^{2}}}\left\{\frac{1}{(y-1) y}\right\}\left\{-\frac{83}{331776 \pi^{2} T^{2}}\right\} \\
& +\langle\bar{q} q\rangle^{2}\left\langle\bar{q} g_{s} \sigma G q\right\rangle^{2} \int_{y_{i}}^{y_{f}} d y e^{-\frac{\tilde{m}^{2}}{T^{2}}}\left\{\frac{83}{663552 \pi^{2}}+\frac{83 \tilde{m}^{2}}{663552 \pi^{2} T^{2}}\right\} \\
& +m_{c}^{2}\left\langle\bar{q} g_{s} \sigma G q\right\rangle^{2}\langle\bar{q} q\rangle^{2} \int_{y_{i}}^{y_{f}} d y e^{-\frac{\tilde{m}^{2}}{T^{2}}}\left\{\frac{1}{y}\right\}\left\{-\frac{25 \tilde{m}^{2}}{497664 \pi^{4} T^{4}}\right\} \\
& +m_{c}^{2}\left\langle\bar{q} g_{s} \sigma G q\right\rangle^{2}\langle\bar{q} q\rangle^{2} \int_{y_{i}}^{y_{f}} d y e^{-\frac{\tilde{m}^{2}}{T^{2}}}\left\{\frac{y}{(y-1)^{2}}\right\}\left\{-\frac{25 \tilde{m}^{2}}{497664 \pi^{4} T^{4}}\right\} \\
& +\left\langle\bar{q} g_{s} \sigma G q\right\rangle^{2}\langle\bar{q} q\rangle^{2} \int_{y_{i}}^{y_{f}} d y e^{-\frac{\tilde{m}^{2}}{T^{2}}}\left\{\frac{y-1}{y}\right\}\left\{\frac{25}{373248 \pi^{4}}+\frac{25 \tilde{m}^{2}}{373248 \pi^{4} T^{2}}-\frac{25 \tilde{m}^{4} y}{746496 \pi^{4} T^{4}}\right\} \\
& +\left\langle\bar{q} g_{s} \sigma G q\right\rangle^{2}\langle\bar{q} q\rangle^{2} \int_{y_{i}}^{y_{f}} d y e^{-\frac{\tilde{m}^{2}}{T^{2}}}\left\{\frac{y-1}{y}\right\}\left\{-\frac{25 \tilde{m}^{2} y}{373248 \pi^{4} T^{2}}-\frac{25 y}{373248 \pi^{4}}\right\} \\
& +\left\langle\bar{q} g_{s} \sigma G q\right\rangle^{2}\langle\bar{q} q\rangle^{2} \int_{y_{i}}^{y_{f}} d y e^{-\frac{\tilde{m}^{2}}{T^{2}}}\left\{\frac{y-1}{y}\right\}\left\{-\frac{25}{1492992 \pi^{4}}-\frac{25 \tilde{m}^{2}}{1492992 \pi^{4} T^{2}}+\frac{25 \tilde{m}^{4} y}{746496 \pi^{4} T^{4}}\right\} \\
& +\left\langle\bar{q} g_{s} \sigma G q\right\rangle^{2}\langle\bar{q} q\rangle^{2} \int_{y_{i}}^{y_{f}} d y e^{-\frac{\tilde{m}^{2}}{T^{2}}}\left\{\frac{y-1}{y}\right\}\left\{\frac{25 \tilde{m}^{2} y}{373248 \pi^{4} T^{2}}+\frac{25 y}{373248 \pi^{4}}\right\} \\
& +m_{c}^{2}\langle\bar{q} q\rangle^{2}\left\langle\bar{q} g_{s} \sigma G q\right\rangle^{2} \int_{y_{i}}^{y_{f}} d y e^{-\frac{\tilde{m}^{2}}{T^{2}}}\left\{\frac{5 \tilde{m}^{4}}{186624 \pi^{4} T^{6}}\right\} \\
& +\langle\bar{q} q\rangle^{2}\left\langle\bar{q} g_{s} \sigma G q\right\rangle^{2} \int_{y_{i}}^{y_{f}} d y e^{-\frac{\tilde{m}^{2}}{T^{2}}}\{(y-1) y\}\left\{-\frac{5}{31104 \pi^{4}}-\frac{5 \tilde{m}^{6}}{186624 \pi^{4} T^{6}}\right\} \\
& +\langle\bar{q} q\rangle^{2}\left\langle\bar{q} g_{s} \sigma G q\right\rangle^{2} \int_{y_{i}}^{y_{f}} d y e^{-\frac{\tilde{m}^{2}}{T^{2}}}\{(y-1) y\}\left\{-\frac{5 \tilde{m}^{4}}{62208 \pi^{4} T^{4}}-\frac{5 \tilde{m}^{2}}{31104 \pi^{4} T^{2}}\right\},
\end{aligned}
$$




$$
\begin{aligned}
& \mathbf{S}_{C}(16)_{2}=2 m_{c}^{2}\langle\bar{q} q\rangle^{4}\left\langle g_{s}^{2} G G\right\rangle \int_{y_{i}}^{y_{f}} d y e^{-\frac{\tilde{m}^{2}}{T^{2}}}\left\{\frac{1}{y^{2}}\right\}\left\{\frac{7}{10368 \pi^{2} T^{2}}\right\} \\
& +m_{c}^{4}\langle\bar{q} q\rangle^{4}\left\langle g_{s}^{2} G G\right\rangle \int_{y_{i}}^{y_{f}} d y e^{-\frac{\tilde{m}^{2}}{T^{2}}}\left\{\frac{1}{y^{3}}\right\}\left\{-\frac{7}{15552 \pi^{2} T^{4}}\right\} \\
& +m_{c}^{2}\langle\bar{q} q\rangle^{4}\left\langle g_{s}^{2} G G\right\rangle \int_{y_{i}}^{y_{f}} d y e^{-\frac{\tilde{m}^{2}}{T^{2}}}\left\{\frac{y-1}{y^{2}}\right\}\left\{\frac{7 \tilde{m}^{2}}{15552 \pi^{2} T^{4}}\right\} \\
& +\left\langle g_{s}^{2} G G\right\rangle\langle\bar{q} q\rangle^{4} \int_{y_{i}}^{y_{f}} d y e^{-\frac{\tilde{m}^{2}}{T^{2}}}\left\{-\frac{7}{41472 \pi^{2}}-\frac{7 \tilde{m}^{2}}{41472 \pi^{2} T^{2}}\right\} \\
& +m_{c}^{2}\left\langle g_{s}^{2} G G\right\rangle\langle\bar{q} q\rangle^{4} \int_{y_{i}}^{y_{f}} d y e^{-\frac{\tilde{m}^{2}}{T^{2}}}\left\{\frac{1}{(y-1) y}\right\}\left\{\frac{7}{20736 \pi^{2} T^{2}}\right\} \\
& +\frac{1}{6} m_{c}^{2}\langle\bar{q} q\rangle^{4}\left\langle g_{s}^{2} G G\right\rangle \int_{y_{i}}^{y_{f}} d y e^{-\frac{\tilde{m}^{2}}{T^{2}}}\left\{\frac{7 \tilde{m}^{4}}{2592 \pi^{2} T^{6}}\right\} \\
& +\frac{1}{6}\langle\bar{q} q\rangle^{4}\left\langle g_{s}^{2} G G\right\rangle \int_{y_{i}}^{y_{f}} d y e^{-\frac{\tilde{m}^{2}}{T^{2}}}\{(y-1) y\}\left\{-\frac{7}{432 \pi^{2}}-\frac{7 \tilde{m}^{6}}{2592 \pi^{2} T^{6}}-\frac{7 \tilde{m}^{4}}{864 \pi^{2} T^{4}}-\frac{7 \tilde{m}^{2}}{432 \pi^{2} T^{2}}\right\} \\
& +2 m_{c}^{2}\left\langle g_{s}^{2} G G\right\rangle\langle\bar{q} q\rangle^{4} \int_{y_{i}}^{y_{f}} d y e^{-\frac{\tilde{m}^{2}}{T^{2}}}\left\{\frac{1}{y^{2}}\right\}\left\{\frac{1}{34992 \pi^{4} T^{2}}\right\} \\
& +m_{c}^{4}\left\langle g_{s}^{2} G G\right\rangle\langle\bar{q} q\rangle^{4} \int_{y_{i}}^{y_{f}} d y e^{-\frac{\tilde{m}^{2}}{T^{2}}}\left\{\frac{1}{y^{3}}\right\}\left\{-\frac{1}{52488 \pi^{4} T^{4}}\right\} \\
& +m_{c}^{2}\left\langle g_{s}^{2} G G\right\rangle\langle\bar{q} q\rangle^{4} \int_{y_{i}}^{y_{f}} d y e^{-\frac{\tilde{m}^{2}}{T^{2}}}\left\{\frac{y-1}{y^{2}}\right\}\left\{\frac{\tilde{m}^{2}}{52488 \pi^{4} T^{4}}\right\} \\
& +2 m_{c}^{2}\left\langle g_{s}^{2} G G\right\rangle\langle\bar{q} q\rangle^{4} \int_{y_{i}}^{y_{f}} d y e^{-\frac{\tilde{m}^{2}}{T^{2}}}\left\{\frac{1}{y^{2}}\right\}\left\{-\frac{1}{93312 \pi^{4} T^{2}}\right\} \\
& +m_{c}^{4}\left\langle g_{s}^{2} G G\right\rangle\langle\bar{q} q\rangle^{4} \int_{y_{i}}^{y_{f}} d y e^{-\frac{\tilde{m}^{2}}{T^{2}}}\left\{\frac{1}{y^{3}}\right\}\left\{\frac{1}{139968 \pi^{4} T^{4}}\right\} \\
& +m_{c}^{2}\left\langle g_{s}^{2} G G\right\rangle\langle\bar{q} q\rangle^{4} \int_{y_{i}}^{y_{f}} d y e^{-\frac{\tilde{m}^{2}}{T^{2}}}\left\{\frac{y-1}{y^{2}}\right\}\left\{-\frac{\tilde{m}^{2}}{139968 \pi^{4} T^{4}}\right\} \\
& +\frac{1}{6} m_{c}^{2}\langle\bar{q} q\rangle^{4}\left\langle g_{s}^{2} G G\right\rangle \int_{y_{i}}^{y_{f}} d y e^{-\frac{\tilde{m}^{2}}{T^{2}}}\left\{\frac{7 \tilde{m}^{4}}{186624 \pi^{4} T^{6}}\right\} \\
& +\frac{1}{6}\langle\bar{q} q\rangle^{4}\left\langle g_{s}^{2} G G\right\rangle \int_{y_{i}}^{y_{f}} d y e^{-\frac{\tilde{m}^{2}}{T^{2}}}\{(y-1) y\}\left\{-\frac{7}{31104 \pi^{4}}-\frac{7 \tilde{m}^{6}}{186624 \pi^{4} T^{6}}-\frac{7 \tilde{m}^{4}}{62208 \pi^{4} T^{4}}-\frac{7 \tilde{m}^{2}}{31104 \pi^{4} T^{2}}\right\} \\
& +2 m_{c}^{2}\langle\bar{q} q\rangle^{4}\left\langle g_{s}^{2} G G\right\rangle \int_{y_{i}}^{y_{f}} d y e^{-\frac{\tilde{m}^{2}}{T^{2}}}\left\{\frac{1}{y^{2}}\right\}\left\{\frac{7}{20155392 \pi^{6} T^{2}}\right\} \\
& +m_{c}^{4}\langle\bar{q} q\rangle^{4}\left\langle g_{s}^{2} G G\right\rangle \int_{y_{i}}^{y_{f}} d y e^{-\frac{\tilde{m}^{2}}{T^{2}}}\left\{\frac{1}{y^{3}}\right\}\left\{-\frac{7}{30233088 \pi^{6} T^{4}}\right\} \\
& +m_{c}^{2}\langle\bar{q} q\rangle^{4}\left\langle g_{s}^{2} G G\right\rangle \int_{y_{i}}^{y_{f}} d y e^{-\frac{\tilde{m}^{2}}{T^{2}}}\left\{\frac{y-1}{y^{2}}\right\}\left\{\frac{7 \tilde{m}^{2}}{30233088 \pi^{6} T^{4}}\right\}
\end{aligned}
$$




$$
\begin{aligned}
\mathbf{S}_{C}(16)_{3} & =2 m_{c}^{2}\left\langle\bar{q} g_{s} \sigma G q\right\rangle\left\langle g_{s}^{2} G G\right\rangle^{2}\langle\bar{q} q\rangle \int_{y_{i}}^{y_{f}} d y e^{-\frac{\tilde{m}^{2}}{T^{2}}}\left\{\frac{1}{y^{2}}\right\}\left\{-\frac{1}{331776 \pi^{6} T^{2}}\right\} \\
& +m_{c}^{4}\left\langle\bar{q} g_{s} \sigma G q\right\rangle\left\langle g_{s}^{2} G G\right\rangle^{2}\langle\bar{q} q\rangle \int_{y_{i}}^{y_{f}} d y e^{-\frac{\tilde{m}^{2}}{T^{2}}}\left\{\frac{1}{y^{3}}\right\}\left\{\frac{1}{497664 \pi^{6} T^{4}}\right\} \\
& +m_{c}^{2}\left\langle\bar{q} g_{s} \sigma G q\right\rangle\left\langle g_{s}^{2} G G\right\rangle^{2}\langle\bar{q} q\rangle \int_{y_{i}}^{y_{f}} d y e^{-\frac{\tilde{m}^{2}}{T^{2}}}\left\{\frac{y-1}{y^{2}}\right\}\left\{-\frac{\tilde{m}^{2}}{497664 \pi^{6} T^{4}}\right\} \\
& +2 m_{c}^{2}\left\langle\bar{q} g_{s} \sigma G q\right\rangle\left\langle g_{s}^{2} G G\right\rangle^{2}\langle\bar{q} q\rangle \int_{y_{i}}^{y_{f}} d y e^{-\frac{\tilde{m}^{2}}{T^{2}}}\left\{\frac{1}{y^{2}}\right\}\left\{\frac{1}{884736 \pi^{6} T^{2}}\right\} \\
& +m_{c}^{4}\left\langle\bar{q} g_{s} \sigma G q\right\rangle\left\langle g_{s}^{2} G G\right\rangle^{2}\langle\bar{q} q\rangle \int_{y_{i}}^{y_{f}} d y e^{-\frac{\tilde{m}^{2}}{T^{2}}}\left\{\frac{1}{y^{3}}\right\}\left\{-\frac{1}{1327104 \pi^{6} T^{4}}\right\} \\
& +m_{c}^{2}\left\langle\bar{q} g_{s} \sigma G q\right\rangle\left\langle g_{s}^{2} G G\right\rangle^{2}\langle\bar{q} q\rangle \int_{y_{i}}^{y_{f}} d y e^{-\frac{\tilde{m}^{2}}{T^{2}}}\left\{\frac{y-1}{y^{2}}\right\}\left\{\frac{\tilde{m}^{2}}{1327104 \pi^{6} T^{4}}\right\} \\
& +m_{c}^{2}\langle\bar{q} q\rangle\left\langle\bar{q} g_{s} \sigma G q\right\rangle\left\langle g_{s}^{2} G G\right\rangle^{2} \int_{y_{i}}^{y_{f}} d y e^{-\frac{\tilde{m}^{2}}{T^{2}}}\left\{\frac{1}{(y-1) y}\right\}\left\{-\frac{145}{84934656 \pi^{6} T^{2}}\right\} \\
& +\langle\bar{q} q\rangle\left\langle\bar{q} g_{s} \sigma G q\right\rangle\left\langle g_{s}^{2} G G\right\rangle^{2} \int_{y_{i}}^{y_{f}} d y e^{-\frac{\tilde{m}^{2}}{T^{2}}}\left\{\frac{259}{254803968 \pi^{6}}+\frac{259 \tilde{m}^{2}}{254803968 \pi^{6} T^{2}}\right\} .
\end{aligned}
$$

Type D for $J_{1}$

$$
\begin{gathered}
\mathbf{S}_{D}(12)_{1} \quad=2 m_{c}^{2}\left\langle\bar{q} g_{s} \sigma G q\right\rangle\langle\bar{q} q\rangle\left\langle g_{s}^{2} G G\right\rangle \int_{y_{i}}^{y_{f}} d y \int_{z_{i}}^{1-y} d z e^{-\frac{\bar{m}^{2}}{T^{2}}}\left\{\frac{y+z-1}{y^{2}}\right\}\left\{-\frac{5 \bar{m}^{2} y}{55296 \pi^{6}}\right\} \\
+2 m_{c}^{2}\left\langle\bar{q} g_{s} \sigma G q\right\rangle\langle\bar{q} q\rangle\left\langle g_{s}^{2} G G\right\rangle \int_{y_{i}}^{y_{f}} d y \int_{z_{i}}^{1-y} d z e^{-\frac{\bar{m}^{2}}{T^{2}}}\left\{-\frac{z(y+z-1)}{y^{2}}\right\}\left\{\frac{5 \bar{m}^{2}}{55296 \pi^{6}}\right\} \\
\mathbf{S}_{D}(12)_{2} \quad=2 m_{c}^{2}\langle\bar{q} q\rangle^{4} \int_{y_{i}}^{y_{f}} d y \int_{z_{i}}^{1-y} d z e^{-\frac{\bar{m}^{2}}{T^{2}}}\left\{\frac{y+z-1}{y}\right\}\left\{\frac{25 \bar{m}^{2}}{62208 \pi^{4}}\right\} \\
+2 m_{c}^{2}\langle\bar{q} q\rangle^{4} \int_{y_{i}}^{y_{f}} d y \int_{z_{i}}^{1-y} d z e^{-\frac{\bar{m}^{2}}{T^{2}}}\left\{-\frac{z(y+z-1)}{y^{2}}\right\}\left\{-\frac{25 \bar{m}^{2}}{62208 \pi^{4}}\right\} \\
+2\langle\bar{q} q\rangle^{4} \int_{y_{i}}^{y_{f}} d y \int_{z_{i}}^{1-y} d z e^{-\frac{\bar{m}^{2}}{T^{2}}}\left\{-\frac{z(y+z-1)}{y}\right\}\left\{\frac{25 \bar{m}^{4} y}{93312 \pi^{4}}\right\} \\
+\langle\bar{q} q\rangle^{4} \int_{y_{i}}^{y_{f}} d y \int_{z_{i}}^{1-y} d z e^{-\frac{\bar{m}^{2}}{T^{2}}}\left\{-\frac{z(y+z-1)}{y}\right\}\left\{-\frac{25 \bar{m}^{4} y}{93312 \pi^{4}}\right\}
\end{gathered}
$$




$$
\begin{aligned}
\mathbf{S}_{D}(12)_{3} & =\langle\bar{\psi} \psi\rangle^{2}\langle\bar{q} q\rangle^{2} \int_{y_{i}}^{y_{f}} d y \int_{z_{i}}^{1-y} d z e^{-\frac{\bar{m}^{2}}{T^{2}}}\left\{-(y+z-1)^{2}\right\}\left\{\frac{35 \bar{m}^{4}}{1119744 \pi^{6}}\right\} \\
& +\langle\bar{\psi} \psi\rangle^{2}\langle\bar{q} q\rangle^{2} m_{c}^{4} \int_{y_{i}}^{y_{f}} d y \int_{z_{i}}^{1-y} d z e^{-\frac{\bar{m}^{2}}{T^{2}}}\left\{-\frac{(y+z-1)^{2}}{y^{2} z^{2}}\right\}\left\{\frac{5}{373248 \pi^{6}}\right\} \\
& +\langle\bar{\psi} \psi\rangle^{2}\langle\bar{q} q\rangle^{2} m_{c}^{2} \int_{y_{i}}^{y_{f}} d y \int_{z_{i}}^{1-y} d z e^{-\frac{\bar{m}^{2}}{T^{2}}}\left\{-\frac{(y+z-1)^{2}}{y z^{2}}\right\}\left\{-\frac{5 \bar{m}^{2} y}{373248 \pi^{6}}\right\} \\
& +\langle\bar{\psi} \psi\rangle^{2}\langle\bar{q} q\rangle^{2} \int_{y_{i}}^{y_{f}} d y \int_{z_{i}}^{1-y} d z e^{-\frac{\bar{m}^{2}}{T^{2}}}\left\{-(y+z-1)^{2}\right\}\left\{-\frac{5 \bar{m}^{4}}{1119744 \pi^{6}}\right\} \\
& +\langle\bar{\psi} \psi\rangle^{2}\langle\bar{q} q\rangle^{2} m_{c}^{2} \int_{y_{i}}^{y_{f}} d y \int_{z_{i}}^{1-y} d z e^{-\frac{\bar{m}^{2}}{T^{2}}}\left\{\frac{(y+z-1)^{2}}{y z}\right\}\left\{-\frac{5 \bar{m}^{2}}{93312 \pi^{6}}\right\}
\end{aligned}
$$

$\mathbf{S}_{D}(12)_{4}=\left\langle g_{s}^{2} G G\right\rangle^{3} \int_{y_{i}}^{y_{f}} d y \int_{z_{i}}^{1-y} d z e^{-\frac{\bar{m}^{2}}{T^{2}}}\left\{-(y+z-1)^{2}\right\}\left\{-\frac{103 \bar{m}^{4}}{195689447424 \pi^{10}}\right\}$

$\mathbf{S}_{D}(12)_{5}=\left\langle g_{s}^{3} f G G G\right\rangle^{2} \int_{y_{i}}^{y_{f}} d y \int_{z_{i}}^{1-y} d z e^{-\frac{\bar{m}^{2}}{T^{2}}}\left\{-(y+z-1)^{2}\right\}\left\{\frac{\bar{m}^{4}}{150994944 \pi^{10}}\right\}$

$$
\begin{aligned}
\mathbf{S}_{D}(14)_{1} & =2 m_{c}^{2}\left\langle\bar{q} g_{s} \sigma G q\right\rangle^{2}\left\langle g_{s}^{2} G G\right\rangle \int_{y_{i}}^{y_{f}} d y \int_{z_{i}}^{1-y} d z e^{-\frac{\bar{m}^{2}}{T^{2}}}\left\{-\frac{1}{y^{2}}\right\}\left\{-\frac{5}{221184 \pi^{6}}+\frac{5 \bar{m}^{2} y}{442368 \pi^{6} T^{2}}+\frac{5 y}{442368 \pi^{6}}\right\} \\
& +2 m_{c}^{2}\left\langle\bar{q} g_{s} \sigma G q\right\rangle^{2}\left\langle g_{s}^{2} G G\right\rangle \int_{y_{i}}^{y_{f}} d y \int_{z_{i}}^{1-y} d z e^{-\frac{\bar{m}^{2}}{T^{2}}}\left\{\frac{z}{y^{2}}\right\}\left\{-\frac{5}{442368 \pi^{6}}-\frac{5 \bar{m}^{2}}{442368 \pi^{6} T^{2}}\right\} \\
& +\left\langle g_{s}^{2} G G\right\rangle\left\langle\bar{q} g_{s} \sigma G q\right\rangle^{2} \int_{y_{i}}^{y_{f}} d y \int_{z_{i}}^{1-y} d z e^{-\frac{\bar{m}^{2}}{T^{2}}}\{-1\}\left\{\frac{5 \bar{m}^{2}}{589824 \pi^{6}}\right\} \\
& +\left\langle g_{s}^{2} G G\right\rangle\left\langle\bar{q} g_{s} \sigma G q\right\rangle^{2} m_{c}^{2} \int_{y_{i}}^{y_{f}} d y \int_{z_{i}}^{1-y} d z e^{-\frac{\bar{m}^{2}}{T^{2}}}\left\{\frac{1}{y z}\right\}\left\{-\frac{5}{294912 \pi^{6}}\right\}
\end{aligned}
$$




$$
\begin{aligned}
& \mathbf{S}_{D}(14)_{2}=\frac{1}{2} m_{c}^{4}\left\langle g_{s}^{2} G G\right\rangle^{2}\langle\bar{q} q\rangle^{2} \int_{y_{i}}^{y_{f}} d y \int_{z_{i}}^{1-y} d z e^{-\frac{\bar{m}^{2}}{T^{2}}}\left\{-\frac{(y+z-1)^{2}}{y^{2} z^{2}}\right\}\left\{-\frac{5 \bar{m}^{2}}{1990656 \pi^{6} T^{4}}\right\} \\
& +m_{c}^{2}\left\langle g_{s}^{2} G G\right\rangle^{2}\langle\bar{q} q\rangle^{2} \int_{y_{i}}^{y_{f}} d y \int_{z_{i}}^{1-y} d z e^{-\frac{\bar{m}^{2}}{T^{2}}}\left\{\frac{(y+z-1)^{3}}{(y-1) y^{2} z^{2}}\right\}\left\{\frac{5}{995328 \pi^{6}}-\frac{5 \bar{m}^{2} y}{1990656 \pi^{6} T^{2}}\right\} \\
& +m_{c}^{2}\left\langle g_{s}^{2} G G\right\rangle^{2}\langle\bar{q} q\rangle^{2} \int_{y_{i}}^{y_{f}} d y \int_{z_{i}}^{1-y} d z e^{-\frac{\bar{m}^{2}}{T^{2}}}\left\{\frac{(y+z-1)^{3}}{(y-1) y^{2} z^{2}}\right\}\left\{-\frac{5 y}{1990656 \pi^{6}}\right\} \\
& +m_{c}^{2}\left\langle g_{s}^{2} G G\right\rangle^{2}\langle\bar{q} q\rangle^{2} \int_{y_{i}}^{y_{f}} d y \int_{z_{i}}^{1-y} d z e^{-\frac{\bar{m}^{2}}{T^{2}}}\left\{\frac{(y+z-1)^{2}}{(y-1) y^{2} z}\right\}\left\{\frac{5}{1990656 \pi^{6}}\left(\frac{\bar{m}^{2}}{T^{2}}-1\right)\right\} \\
& +\frac{1}{2} m_{c}^{2}\left\langle g_{s}^{2} G G\right\rangle^{2}\langle\bar{q} q\rangle^{2} \int_{y_{i}}^{y_{f}} d y \int_{z_{i}}^{1-y} d z e^{-\frac{\bar{m}^{2}}{T^{2}}}\left\{\frac{(y+z-1)^{2}}{y z}\right\}\left\{\frac{5}{995328 \pi^{6}}+\frac{5 \bar{m}^{4}}{1990656 \pi^{6} T^{4}}\right\} \\
& +\frac{1}{2} m_{c}^{2}\left\langle g_{s}^{2} G G\right\rangle^{2}\langle\bar{q} q\rangle^{2} \int_{y_{i}}^{y_{f}} d y \int_{z_{i}}^{1-y} d z e^{-\frac{\bar{m}^{2}}{T^{2}}}\left\{\frac{(y+z-1)^{2}}{y z}\right\}\left\{\frac{5 \bar{m}^{2}}{995328 \pi^{6} T^{2}}\right\} \\
& +2 m_{c}^{2}\left\langle g_{s}^{2} G G\right\rangle^{2}\langle\bar{q} q\rangle^{2} \int_{y_{i}}^{y_{f}} d y \int_{z_{i}}^{1-y} d z e^{-\frac{\bar{m}^{2}}{T^{2}}}\left\{\frac{z}{y^{2}}\right\}\left\{\frac{1}{248832 \pi^{6}}-\frac{\bar{m}^{2}}{82944 \pi^{6} T^{2}}\right\} \\
& +2 m_{c}^{2}\left\langle g_{s}^{2} G G\right\rangle^{2}\langle\bar{q} q\rangle^{2} \int_{y_{i}}^{y_{f}} d y \int_{z_{i}}^{1-y} d z e^{-\frac{\bar{m}^{2}}{T^{2}}}\left\{-\frac{1}{y^{2}}\right\}\left\{-\frac{1}{124416 \pi^{6}}+\frac{\bar{m}^{2} y}{248832 \pi^{6} T^{2}}+\frac{y}{248832 \pi^{6}}\right\} \\
& +2 m_{c}^{2}\left\langle g_{s}^{2} G G\right\rangle^{2}\langle\bar{q} q\rangle^{2} \int_{y_{i}}^{y_{f}} d y \int_{z_{i}}^{1-y} d z e^{-\frac{\bar{m}^{2}}{T^{2}}}\left\{\frac{z}{y^{2}}\right\}\left\{\frac{1}{663552 \pi^{6}}+\frac{\bar{m}^{2}}{663552 \pi^{6} T^{2}}\right\} \\
& +2 m_{c}^{2}\left\langle g_{s}^{2} G G\right\rangle^{2}\langle\bar{q} q\rangle^{2} \int_{y_{i}}^{y_{f}} d y \int_{z_{i}}^{1-y} d z e^{-\frac{\bar{m}^{2}}{T^{2}}}\left\{-\frac{1}{y^{2}}\right\}\left\{\frac{1}{331776 \pi^{6}}-\frac{\bar{m}^{2} y}{663552 \pi^{6} T^{2}}-\frac{y}{663552 \pi^{6}}\right\} \\
& +m_{c}^{2}\left\langle g_{s}^{2} G G\right\rangle^{2}\langle\bar{q} q\rangle^{2} \int_{y_{i}}^{y_{f}} d y \int_{z_{i}}^{1-y} d z e^{-\frac{\bar{m}^{2}}{T^{2}}}\left\{\frac{1}{y z}\right\}\left\{-\frac{145}{42467328 \pi^{6}}\right\} \\
& +\left\langle g_{s}^{2} G G\right\rangle^{2}\langle\bar{q} q\rangle^{2} \int_{y_{i}}^{y_{f}} d y \int_{z_{i}}^{1-y} d z e^{-\frac{\bar{m}^{2}}{T^{2}}}\{-1\}\left\{-\frac{259 \bar{m}^{2}}{127401984 \pi^{6}}\right\} \\
& +\left\langle g_{s}^{2} G G\right\rangle^{2}\langle\bar{q} q\rangle^{2} \int_{y_{i}}^{y_{f}} d y \int_{z_{i}}^{1-y} d z e^{-\frac{\bar{m}^{2}}{T^{2}}}\{y+z-1\}\left\{-\frac{83 \bar{m}^{2}}{15925248 \pi^{6}}-\frac{83 \bar{m}^{4}}{63700992 \pi^{6} T^{2}}\right\} \\
& +m_{c}^{2}\left\langle g_{s}^{2} G G\right\rangle^{2}\langle\bar{q} q\rangle^{2} \int_{y_{i}}^{y_{f}} d y \int_{z_{i}}^{1-y} d z e^{-\frac{\bar{m}^{2}}{T^{2}}}\left\{\frac{(y+z-1)^{3}}{(y-1) y^{2} z^{2}}\right\}\left\{\frac{7}{53747712 \pi^{8}}-\frac{7 \bar{m}^{2} y}{107495424 \pi^{8} T^{2}}\right\} \\
& +m_{c}^{2}\left\langle g_{s}^{2} G G\right\rangle^{2}\langle\bar{q} q\rangle^{2} \int_{y_{i}}^{y_{f}} d y \int_{z_{i}}^{1-y} d z e^{-\frac{\bar{m}^{2}}{T^{2}}}\left\{\frac{(y+z-1)^{3}}{(y-1) y^{2} z^{2}}\right\}\left\{-\frac{7 y}{107495424 \pi^{8}}\right\} \\
& +m_{c}^{2}\left\langle g_{s}^{2} G G\right\rangle^{2}\langle\bar{q} q\rangle^{2} \int_{y_{i}}^{y_{f}} d y \int_{z_{i}}^{1-y} d z e^{-\frac{\bar{m}^{2}}{T^{2}}}\left\{\frac{(y+z-1)^{2}}{(y-1) y^{2} z}\right\}\left\{\frac{7 \bar{m}^{2}}{107495424 \pi^{8} T^{2}}-\frac{7}{107495424 \pi^{8}}\right\} \\
& +\frac{1}{2} m_{c}^{2}\left\langle g_{s}^{2} G G\right\rangle^{2}\langle\bar{q} q\rangle^{2} \int_{y_{i}}^{y_{f}} d y \int_{z_{i}}^{1-y} d z e^{-\frac{\bar{m}^{2}}{T^{2}}}\left\{\frac{(y+z-1)^{2}}{y z}\right\}\left\{\frac{7}{53747712 \pi^{8}}+\frac{7 \bar{m}^{4}}{107495424 \pi^{8} T^{4}}\right\} \\
& +\frac{1}{2} m_{c}^{2}\left\langle g_{s}^{2} G G\right\rangle^{2}\langle\bar{q} q\rangle^{2} \int_{y_{i}}^{y_{f}} d y \int_{z_{i}}^{1-y} d z e^{-\frac{\bar{m}^{2}}{T^{2}}}\left\{\frac{(y+z-1)^{2}}{y z}\right\}\left\{\frac{7 \bar{m}^{2}}{53747712 \pi^{8} T^{2}}\right\} \\
& +\frac{1}{2} m_{c}^{4}\left\langle g_{s}^{2} G G\right\rangle^{2}\langle\bar{q} q\rangle^{2} \int_{y_{i}}^{y_{f}} d y \int_{z_{i}}^{1-y} d z e^{-\frac{\bar{m}^{2}}{T^{2}}}\left\{-\frac{(y+z-1)^{2}}{y^{2} z^{2}}\right\}\left\{-\frac{7 \bar{m}^{2}}{107495424 \pi^{8} T^{4}}\right\},
\end{aligned}
$$




$$
\begin{aligned}
\mathbf{S}_{D}(14)_{3} & =2 m_{c}^{2}\langle\bar{q} q\rangle^{3}\left\langle\bar{q} g_{s} \sigma G q\right\rangle \int_{y_{i}}^{y_{f}} d y \int_{z_{i}}^{1-y} d z e^{-\frac{\bar{m}^{2}}{T^{2}}}\left\{-\frac{1}{y}\right\}\left\{-\frac{25}{124416 \pi^{4}}-\frac{25 \bar{m}^{2}}{124416 \pi^{4} T^{2}}\right\} \\
& +2 m_{c}^{2}\langle\bar{q} q\rangle^{3}\left\langle\bar{q} g_{s} \sigma G q\right\rangle \int_{y_{i}}^{y_{f}} d y \int_{z_{i}}^{1-y} d z e^{-\frac{\bar{m}^{2}}{T^{2}}}\left\{\frac{z}{y^{2}}\right\}\left\{\frac{25}{124416 \pi^{4}}+\frac{25 \bar{m}^{2}}{124416 \pi^{4} T^{2}}\right\} \\
& +2\left\langle\bar{q} g_{s} \sigma G q\right\rangle\langle\bar{q} q\rangle^{3} \int_{y_{i}}^{y_{f}} d y \int_{z_{i}}^{1-y} d z e^{-\frac{\bar{m}^{2}}{T^{2}}}\left\{\frac{z}{y}\right\}\left\{\frac{25 \bar{m}^{2}}{93312 \pi^{4}}-\frac{25 \bar{m}^{4} y}{186624 \pi^{4} T^{2}}-\frac{25 \bar{m}^{2} y}{46656 \pi^{4}}\right\} \\
& +2\left\langle\bar{q} g_{s} \sigma G q\right\rangle\langle\bar{q} q\rangle^{3} \int_{y_{i}}^{y_{f}} d y \int_{z_{i}}^{1-y} d z e^{-\frac{\bar{m}^{2}}{T^{2}}}\left\{\frac{z}{y}\right\}\left\{-\frac{25 \bar{m}^{2}}{373248 \pi^{4}}+\frac{25 \bar{m}^{4} y}{186624 \pi^{4} T^{2}}+\frac{25 \bar{m}^{2} y}{46656 \pi^{4}}\right\},
\end{aligned}
$$

$$
\begin{aligned}
\mathbf{S}_{D}(14)_{4} & =m_{c}^{4}\left\langle\bar{q} g_{s} \sigma G q\right\rangle\langle\bar{q} q\rangle\langle\bar{\psi} \psi\rangle^{2} \int_{y_{i}}^{y_{f}} d y \int_{z_{i}}^{1-y} d z e^{-\frac{\bar{m}^{2}}{T^{2}}}\left\{\frac{y+z-1}{y^{2} z^{2}}\right\}\left\{-\frac{5}{373248 \pi^{6} T^{2}}\right\} \\
& +2 m_{c}^{2}\left\langle\bar{q} g_{s} \sigma G q\right\rangle\langle\bar{q} q\rangle\langle\bar{\psi} \psi\rangle^{2} \int_{y_{i}}^{y_{f}} d y \int_{z_{i}}^{1-y} d z e^{-\frac{\bar{m}^{2}}{T^{2}}}\left\{\frac{y+z-1}{y z^{2}}\right\}\left\{-\frac{5}{373248 \pi^{6}}+\frac{5 \bar{m}^{2} y}{746496 \pi^{6} T^{2}}\right\} \\
& +2 m_{c}^{2}\left\langle\bar{q} g_{s} \sigma G q\right\rangle\langle\bar{q} q\rangle\langle\bar{\psi} \psi\rangle^{2} \int_{y_{i}}^{y_{f}} d y \int_{z_{i}}^{1-y} d z e^{-\frac{\bar{m}^{2}}{T^{2}}}\left\{\frac{y+z-1}{y z^{2}}\right\}\left\{\frac{5 y}{746496 \pi^{6}}\right\} \\
& +\left\langle\bar{q} g_{s} \sigma G q\right\rangle\langle\bar{q} q\rangle\langle\bar{\psi} \psi\rangle^{2} m_{c}^{2} \int_{y_{i}}^{y_{f}} d y \int_{z_{i}}^{1-y} d z e^{-\frac{\bar{m}^{2}}{T^{2}}}\left\{-\frac{y+z-1}{y z}\right\}\left\{\frac{5}{93312 \pi^{6}}+\frac{5 \bar{m}^{2}}{93312 \pi^{6} T^{2}}\right\} \\
& +\left\langle\bar{q} g_{s} \sigma G q\right\rangle\langle\bar{q} q\rangle\langle\bar{\psi} \psi\rangle^{2} \int_{y_{i}}^{y_{f}} d y \int_{z_{i}}^{1-y} d z e^{-\frac{\bar{m}^{2}}{T^{2}}}\left\{\frac{(y+z-1)^{2}}{(y-1) y z}\right\}\left\{-\frac{5 \bar{m}^{2} y}{559872 \pi^{6}}\right\} \\
& +\left\langle\bar{q} g_{s} \sigma G q\right\rangle\langle\bar{q} q\rangle\langle\bar{\psi} \psi\rangle^{2} \int_{y_{i}}^{y_{f}} d y \int_{z_{i}}^{1-y} d z e^{-\frac{\bar{m}^{2}}{T^{2}}}\left\{\frac{y+z-1}{(y-1) y}\right\}\left\{\frac{5 \bar{m}^{2}}{559872 \pi^{6}}\right\} \\
& +\left\langle\bar{q} g_{s} \sigma G q\right\rangle\langle\bar{q} q\rangle\langle\bar{\psi} \psi\rangle^{2} \int_{y_{i}}^{y_{f}} d y \int_{z_{i}}^{1-y} d z e^{-\frac{\bar{m}^{2}}{T^{2}}}\{y+z-1\}\left\{\frac{5 \bar{m}^{2}}{279936 \pi^{6}}+\frac{5 \bar{m}^{4}}{1119744 \pi^{6} T^{2}}\right\} \\
& +\left\langle\bar{q} g_{s} \sigma G q\right\rangle\langle\bar{q} q\rangle\langle\bar{\psi} \psi\rangle^{2} \int_{y_{i}}^{y_{f}} d y \int_{z_{i}}^{1-y} d z e^{-\frac{\bar{m}^{2}}{T^{2}}}\left\{\frac{(y+z-1)^{2}}{(y-1) y z}\right\}\left\{\frac{35 \bar{m}^{2} y}{2239488 \pi^{6}}\right\} \\
& +\left\langle\bar{q} g_{s} \sigma G q\right\rangle\langle\bar{q} q\rangle\langle\bar{\psi} \psi\rangle^{2} \int_{y_{i}}^{y_{f}} d y \int_{z_{i}}^{1-y} d z e^{-\frac{\bar{m}^{2}}{T^{2}}}\left\{\frac{y+z-1}{(y-1) y}\right\}\left\{-\frac{35 \bar{m}^{2}}{2239488 \pi^{6}}\right\} \\
& +\left\langle\bar{q} g_{s} \sigma G q\right\rangle\langle\bar{q} q\rangle\langle\bar{\psi} \psi\rangle^{2} \int_{y_{i}}^{y_{f}} d y \int_{z_{i}}^{1-y} d z e^{-\frac{\bar{m}^{2}}{T^{2}}}\{y+z-1\}\left\{-\frac{35 \bar{m}^{2}}{279936 \pi^{6}}-\frac{35 \bar{m}^{4}}{1119744 \pi^{6} T^{2}}\right\}
\end{aligned}
$$




$$
\begin{aligned}
\mathbf{S}_{D}(16)_{1}= & \frac{1}{2} m_{c}^{2}\langle\bar{q} q\rangle\left\langle\bar{q} g_{s} \sigma G q\right\rangle\left\langle g_{s}^{2} G G\right\rangle^{2} \int_{y_{i}}^{y_{f}} d y \int_{z_{i}}^{1-y} d z e^{-\frac{\bar{m}^{2}}{T^{2}}}\left\{-\frac{(y+z-1)^{2}}{(y-1) y^{2} z^{2}}\right\}\left\{\frac{5 \bar{m}^{2} y}{995328 \pi^{6} T^{4}}\right\} \\
& +\frac{1}{2} m_{c}^{2}\langle\bar{q} q\rangle\left\langle\bar{q} g_{s} \sigma G q\right\rangle\left\langle g_{s}^{2} G G\right\rangle^{2} \int_{y_{i}}^{y_{f}} d y \int_{z_{i}}^{1-y} d z e^{-\frac{\bar{m}^{2}}{T^{2}}}\left\{-\frac{(y+z-1)^{2}}{(y-1) y^{2} z^{2}}\right\}\left\{-\frac{5}{497664 \pi^{6} T^{2}}\right\} \\
& +\frac{1}{2} m_{c}^{2}\langle\bar{q} q\rangle\left\langle\bar{q} g_{s} \sigma G q\right\rangle\left\langle g_{s}^{2} G G\right\rangle^{2} \int_{y_{i}}^{y_{f}} d y \int_{z_{i}}^{1-y} d z e^{-\frac{\bar{m}^{2}}{T^{2}}}\left\{\frac{y+z-1}{y^{2} z(1-y)}\right\}\left\{\frac{5}{497664 \pi^{6} T^{2}}-\frac{5 \bar{m}^{2}}{995328 \pi^{6} T^{4}}\right\} \\
& +\frac{1}{6} m_{c}^{2}\langle\bar{q} q\rangle\left\langle\bar{q} g_{s} \sigma G q\right\rangle\left\langle g_{s}^{2} G G\right\rangle^{2} \int_{y_{i}}^{y_{f}} d y \int_{z_{i}}^{1-y} d z e^{-\frac{\bar{m}^{2}}{T^{2}}}\left\{-\frac{y+z-1}{y z}\right\}\left\{-\frac{5 \bar{m}^{4}}{663552 \pi^{6} T^{6}}\right\} \\
& +\frac{1}{6} m_{c}^{4}\langle\bar{q} q\rangle\left\langle\bar{q} g_{s} \sigma G q\right\rangle\left\langle g_{s}^{2} G G\right\rangle^{2} \int_{y_{i}}^{y_{f}} d y \int_{z_{i}}^{1-y} d z e^{-\frac{\bar{m}^{2}}{T^{2}}}\left\{\frac{y+z-1}{y^{2} z^{2}}\right\}\left\{\frac{5 \bar{m}^{2}}{663552 \pi^{6} T^{6}}-\frac{5}{663552 \pi^{6} T^{4}}\right\} \\
& +\frac{1}{2}\langle\bar{q} q\rangle\left\langle\bar{q} g_{s} \sigma G q\right\rangle\left\langle g_{s}^{2} G G\right\rangle^{2} \int_{y_{i}}^{y_{f}} d y \int_{z_{i}}^{1-y} d z e^{-\frac{\bar{m}^{2}}{T^{2}}}\{-1\}\left\{\frac{83}{31850496 \pi^{6}}+\frac{83 \bar{m}^{4}}{63700992 \pi^{6} T^{4}}\right\} \\
& +\frac{1}{2}\langle\bar{q} q\rangle\left\langle\bar{q} g_{s} \sigma G q\right\rangle\left\langle g_{s}^{2} G G\right\rangle^{2} \int_{y_{i}}^{y_{f}} d y \int_{z_{i}}^{1-y} d z e^{-\frac{\bar{m}^{2}}{T^{2}}}\{-1\}\left\{\frac{83 \bar{m}^{2}}{31850496 \pi^{6} T^{2}}\right\}
\end{aligned}
$$

$$
\begin{aligned}
\mathbf{S}_{D}(16)_{2}= & \frac{1}{2} m_{c}^{4}\left\langle\bar{q} g_{s} \sigma G q\right\rangle^{2}\langle\bar{\psi} \psi\rangle^{2} \int_{y_{i}}^{y_{f}} d y \int_{z_{i}}^{1-y} d z e^{-\frac{\bar{m}^{2}}{T^{2}}}\left\{-\frac{1}{y^{2} z^{2}}\right\}\left\{\frac{5}{1492992 \pi^{6} T^{4}}\right\} \\
& +\frac{1}{2} m_{c}^{2}\left\langle\bar{q} g_{s} \sigma G q\right\rangle^{2}\langle\bar{\psi} \psi\rangle^{2} \int_{y_{i}}^{y_{f}} d y \int_{z_{i}}^{1-y} d z e^{-\frac{\bar{m}^{2}}{T^{2}}}\left\{-\frac{1}{y z^{2}}\right\}\left\{\frac{5}{1492992 \pi^{6} T^{2}}-\frac{5 \bar{m}^{2} y}{2985984 \pi^{6} T^{4}}\right\} \\
& +\frac{1}{2} m_{c}^{2}\left\langle\bar{q} g_{s} \sigma G q\right\rangle^{2}\langle\bar{\psi} \psi\rangle^{2} \int_{y_{i}}^{y_{f}} d y \int_{z_{i}}^{1-y} d z e^{-\frac{\bar{m}^{2}}{T^{2}}}\left\{-\frac{1}{y z^{2}}\right\}\left\{\frac{5}{1492992 \pi^{6} T^{2}}-\frac{5 \bar{m}^{2} y}{2985984 \pi^{6} T^{4}}\right\} \\
& +\frac{1}{2} m_{c}^{2}\left\langle\bar{q} g_{s} \sigma G q\right\rangle^{2}\langle\bar{\psi} \psi\rangle^{2} \int_{y_{i}}^{y_{f}} d y \int_{z_{i}}^{1-y} d z e^{-\frac{\bar{m}^{2}}{T^{2}}}\left\{\frac{1}{y z}\right\}\left\{\frac{5 \bar{m}^{2}}{373248 \pi^{6} T^{4}}\right\} \\
& +\left\langle\bar{q} g_{s} \sigma G q\right\rangle^{2}\langle\bar{\psi} \psi\rangle^{2} \int_{y_{i}}^{y_{f}} d y \int_{z_{i}}^{1-y} d z e^{-\frac{\bar{m}^{2}}{T^{2}}}\left\{\frac{y+z-1}{y z(1-y)}\right\}\left\{-\frac{5}{2239488 \pi^{6}}+\frac{5 \bar{m}^{2} y}{4478976 \pi^{6} T^{2}}+\frac{5 y}{4478976 \pi^{6}}\right\} \\
& +\left\langle\bar{q} g_{s} \sigma G q\right\rangle^{2}\langle\bar{\psi} \psi\rangle^{2} \int_{y_{i}}^{y_{f}} d y \int_{z_{i}}^{1-y} d z e^{-\frac{\bar{m}^{2}}{T^{2}}}\left\{\frac{1}{y-y^{2}}\right\}\left\{\frac{5}{4478976 \pi^{6}}-\frac{5 \bar{m}^{2}}{4478976 \pi^{6} T^{2}}\right\} \\
& +\frac{1}{2}\left\langle\bar{q} g_{s} \sigma G q\right\rangle^{2}\langle\bar{\psi} \psi\rangle^{2} \int_{y_{i}}^{y_{f}} d y \int_{z_{i}}^{1-y} d z e^{-\frac{\bar{m}^{2}}{T^{2}}}\{-1\}\left\{-\frac{5}{2239488 \pi^{6}}-\frac{5 \bar{m}^{4}}{4478976 \pi^{6} T^{4}}-\frac{5 \bar{m}^{2}}{2239488 \pi^{6} T^{2}}\right\} \\
& +\left\langle\bar{q} g_{s} \sigma G q\right\rangle^{2}\langle\bar{\psi} \psi\rangle^{2} \int_{y_{i}}^{y_{f}} d y \int_{z_{i}}^{1-y} d z e^{-\frac{\bar{m}^{2}}{T^{2}}}\left\{\frac{y+z-1}{y z(1-y)}\right\}\left\{\frac{35}{8957952 \pi^{6}}-\frac{35 \bar{m}^{2} y}{17915904 \pi^{6} T^{2}}-\frac{35 y}{17915904 \pi^{6}}\right\} \\
& +\left\langle\bar{q} g_{s} \sigma G q\right\rangle^{2}\langle\bar{\psi} \psi\rangle^{2} \int_{y_{i}}^{y_{f}} d y \int_{z_{i}}^{1-y} d z e^{-\frac{\bar{m}^{2}}{T^{2}}}\left\{\frac{1}{y-y^{2}}\right\}\left\{\frac{35 \bar{m}^{2}}{17915904 \pi^{6} T^{2}}-\frac{35}{17915904 \pi^{6}}\right\} \\
& +\frac{1}{2}\left\langle\bar{q} g_{s} \sigma G q\right\rangle^{2}\langle\bar{\psi} \psi\rangle^{2} \int_{y_{i}}^{y_{f}} d y \int_{z_{i}}^{1-y} d z e^{-\frac{\bar{m}^{2}}{T^{2}}}\{-1\}\left\{\frac{35}{2239488 \pi^{6}}+\frac{35 \bar{m}^{4}}{4478976 \pi^{6} T^{4}}+\frac{35 \bar{m}^{2}}{2239488 \pi^{6} T^{2}}\right\}
\end{aligned}
$$


Type A for $J_{2}$

$$
\begin{aligned}
\mathbf{S}_{A}(12)_{1} & =m_{c}^{2}\langle\bar{q} q\rangle^{4} \int_{4 m_{c}^{2}}^{s_{0}} d s \int_{y_{i}}^{y_{f}} d y e^{-\frac{s}{T^{2}}}\left\{\frac{1}{144 \pi^{2}}\right\} \\
& +\langle\bar{q} q\rangle^{4} \int_{4 m_{c}^{2}}^{s_{0}} d s \int_{y_{i}}^{y_{f}} d y e^{-\frac{s}{T^{2}}}\{(y-1) y\}\left\{\frac{\tilde{m}^{2}-s}{72 \pi^{2}}-\frac{s}{144 \pi^{2}}\right\} \\
& +\langle\bar{q} q\rangle^{4} \int_{4 m_{c}^{2}}^{s_{0}} d s \int_{y_{i}}^{y_{f}} d y e^{-\frac{s}{T^{2}}}\{(y-1) y\}\left\{\frac{\tilde{m}^{2}-s}{1944 \pi^{4}}-\frac{s}{3888 \pi^{4}}\right\} \\
& +m_{c}^{2}\langle\bar{q} q\rangle^{4} \int_{4 m_{c}^{2}}^{s_{0}} d s \int_{y_{i}}^{y_{f}} d y e^{-\frac{s}{T^{2}}}\left\{\frac{1}{3888 \pi^{4}}\right\}
\end{aligned}
$$

$$
\begin{aligned}
\mathbf{S}_{A}(12)_{1}= & m_{c}^{2}\langle\bar{q} q\rangle\left\langle\bar{q} g_{s} \sigma G q\right\rangle\left\langle g_{s}^{2} G G\right\rangle \int_{4 m_{c}^{2}}^{s_{0}} d s \int_{y_{i}}^{y_{f}} d y e^{-\frac{s}{T^{2}}}\left\{-\frac{1}{12288 \pi^{6}}\right\} \\
& +\langle\bar{q} q\rangle\left\langle\bar{q} g_{s} \sigma G q\right\rangle\left\langle g_{s}^{2} G G\right\rangle \int_{4 m_{c}^{2}}^{s_{0}} d s \int_{y_{i}}^{y_{f}} d y e^{-\frac{s}{T^{2}}}\{(y-1) y\}\left\{\frac{s}{12288 \pi^{6}}-\frac{\tilde{m}^{2}-s}{6144 \pi^{6}}\right\} \\
& +m_{c}^{2}\left\langle\bar{q} g_{s} \sigma G q\right\rangle\langle\bar{q} q\rangle\left\langle g_{s}^{2} G G\right\rangle \int_{4 m_{c}^{2}}^{s_{0}} d s \int_{y_{i}}^{y_{f}} d y e^{-\frac{s}{T^{2}}}\left\{-\frac{1}{36864 \pi^{6}}\right\} \\
& +\left\langle\bar{q} g_{s} \sigma G q\right\rangle\langle\bar{q} q\rangle\left\langle g_{s}^{2} G G\right\rangle \int_{4 m_{c}^{2}}^{s_{0}} d s \int_{y_{i}}^{y_{f}} d y e^{-\frac{s}{T^{2}}}\{(y-1) y\}\left\{\frac{s}{36864 \pi^{6}}-\frac{\tilde{m}^{2}-s}{18432 \pi^{6}}\right\}
\end{aligned}
$$

$$
\begin{aligned}
\mathbf{S}_{A}(14)_{1} \quad & =\langle\bar{q} q\rangle^{3}\left\langle\bar{q} g_{s} \sigma G q\right\rangle \int_{4 m_{c}^{2}}^{s_{0}} d s \int_{y_{i}}^{y_{f}} d y e^{-\frac{s}{T^{2}}}\{(y-1) y\}\left\{\frac{1}{24 \pi^{2}}\right\} \\
& +\langle\bar{q} q\rangle^{3}\left\langle\bar{q} g_{s} \sigma G q\right\rangle \int_{4 m_{c}^{2}}^{s_{0}} d s \int_{y_{i}}^{y_{f}} d y e^{-\frac{s}{T^{2}}}\{(y-1) y\}\left\{\frac{1}{1296 \pi^{4}}\right\} .
\end{aligned}
$$

Type B for $J_{2}$

$$
\begin{aligned}
\mathbf{S}_{B}(0) & =m_{c}^{2} \int_{4 m_{c}^{2}}^{s_{0}} d s \int_{y_{i}}^{y_{f}} d y \int_{z_{i}}^{1-y} d z e^{-\frac{s}{T^{2}}}\left\{-(y+z-1)^{5}\right\}\left\{\frac{\left(s-\bar{m}^{2}\right)^{6}}{39321600 \pi^{10}}\right\} \\
& +\int_{4 m_{c}^{2}}^{s_{0}} d s \int_{y_{i}}^{y_{f}} d y \int_{z_{i}}^{1-y} d z e^{-\frac{s}{T^{2}}}\left\{-y z(y+z-1)^{5}\right\}\left\{\frac{\left(s-\bar{m}^{2}\right)^{7}}{137625600 \pi^{10}}+\frac{s\left(s-\bar{m}^{2}\right)^{6}}{39321600 \pi^{10}}\right\}
\end{aligned}
$$




$$
\begin{aligned}
\mathbf{S}_{B}(4) & =m_{c}^{2}\left\langle g_{s}^{2} G G\right\rangle \int_{4 m_{c}^{2}}^{s_{0}} d s \int_{y_{i}}^{y_{f}} d y \int_{z_{i}}^{1-y} d z e^{-\frac{s}{T^{2}}}\left\{\frac{(y+z-1)^{5}}{y^{2}}\right\}\left\{\frac{(y-1)\left(s-\bar{m}^{2}\right)^{4}}{23592960 \pi^{10}}+\frac{s y\left(s-\bar{m}^{2}\right)^{3}}{11796480 \pi^{10}}\right\} \\
& +m_{c}^{2}\left\langle g_{s}^{2} G G\right\rangle \int_{4 m_{c}^{2}}^{s_{0}} d s \int_{y_{i}}^{y_{f}} d y \int_{z_{i}}^{1-y} d z e^{-\frac{s}{T^{2}}}\left\{-\frac{z(y+z-1)^{5}}{y^{2}}\right\}\left\{-\frac{\left(s-\bar{m}^{2}\right)^{4}}{23592960 \pi^{10}}-\frac{s\left(s-\bar{m}^{2}\right)^{3}}{11796480 \pi^{10}}\right\} \\
& +m_{c}^{2}\left\langle g_{s}^{2} G G\right\rangle \int_{4 m_{c}^{2}}^{s_{0}} d s \int_{y_{i}}^{y_{f}} d y \int_{z_{i}}^{1-y} d z e^{-\frac{s}{T^{2}}}\left\{-(y+z-1)^{3}\right\}\left\{\frac{\left(s-\bar{m}^{2}\right)^{4}}{1572864 \pi^{10}}\right\} \\
& +\left\langle g_{s}^{2} G G\right\rangle \int_{4 m_{c}^{2}}^{s_{0}} d s \int_{y_{i}}^{y_{f}} d y \int_{z_{i}}^{1-y} d z e^{-\frac{s}{T^{2}}}\left\{-y z(y+z-1)^{3}\right\}\left\{\frac{\left(s-\bar{m}^{2}\right)^{5}}{3932160 \pi^{10}}+\frac{s\left(s-\bar{m}^{2}\right)^{4}}{1572864 \pi^{10}}\right\},
\end{aligned}
$$

$$
\begin{aligned}
& \mathbf{S}_{B}(6)=m_{c}^{2}\langle\bar{q} q\rangle^{2} \int_{4 m_{c}^{2}}^{s_{0}} d s \int_{y_{i}}^{y_{f}} d y \int_{z_{i}}^{1-y} d z e^{-\frac{s}{T^{2}}}\left\{(y+z-1)^{2}\right\}\left\{\frac{\left(s-\bar{m}^{2}\right)^{3}}{1536 \pi^{6}}\right\} \\
& +\langle\bar{q} q\rangle^{2} \int_{4 m_{c}^{2}}^{s_{0}} d s \int_{y_{i}}^{y_{f}} d y \int_{z_{i}}^{1-y} d z e^{-\frac{s}{T^{2}}}\left\{y z(y+z-1)^{2}\right\}\left\{\frac{\left(s-\bar{m}^{2}\right)^{4}}{3072 \pi^{6}}+\frac{s\left(s-\bar{m}^{2}\right)^{3}}{1536 \pi^{6}}\right\} \\
& +m_{c}^{2}\langle\bar{q} q\rangle^{2} \int_{4 m_{c}^{2}}^{s_{0}} d s \int_{y_{i}}^{y_{f}} d y \int_{z_{i}}^{1-y} d z e^{-\frac{s}{T^{2}}}\left\{(y+z-1)^{2}\right\}\left\{\frac{\left(s-\bar{m}^{2}\right)^{3}}{27648 \pi^{8}}\right\} \\
& +\langle\bar{q} q\rangle^{2} \int_{4 m_{c}^{2}}^{s_{0}} d s \int_{y_{i}}^{y_{f}} d y \int_{z_{i}}^{1-y} d z e^{-\frac{s}{T^{2}}}\left\{y z(y+z-1)^{2}\right\}\left\{\frac{\left(s-\bar{m}^{2}\right)^{4}}{165888 \pi^{8}}+\frac{s\left(s-\bar{m}^{2}\right)^{3}}{82944 \pi^{8}}\right\} \\
& +m_{c}^{2}\langle\bar{q} q\rangle^{2} \int_{4 m_{c}^{2}}^{s_{0}} d s \int_{y_{i}}^{y_{f}} d y \int_{z_{i}}^{1-y} d z e^{-\frac{s}{T^{2}}}\left\{\frac{(y+z-1)^{4}}{y}\right\}\left\{-\frac{\left(s-\bar{m}^{2}\right)^{3}}{331776 \pi^{8}}-\frac{s\left(s-\bar{m}^{2}\right)^{2}}{221184 \pi^{8}}\right\} \\
& +m_{c}^{2}\langle\bar{q} q\rangle^{2} \int_{4 m_{c}^{2}}^{s_{0}} d s \int_{y_{i}}^{y_{f}} d y \int_{z_{i}}^{1-y} d z e^{-\frac{s}{T^{2}}}\left\{\frac{z(y+z-1)^{4}}{y^{2}}\right\}\left\{-\frac{\left(s-\bar{m}^{2}\right)^{3}}{331776 \pi^{8}}-\frac{s\left(s-\bar{m}^{2}\right)^{2}}{221184 \pi^{8}}\right\} \\
& +\langle\bar{q} q\rangle^{2} \int_{4 m_{c}^{2}}^{s_{0}} d s \int_{y_{i}}^{y_{f}} d y \int_{z_{i}}^{1-y} d z e^{-\frac{s}{T^{2}}}\left\{\frac{z(y+z-1)^{4}}{y}\right\}\left\{\frac{s^{2} y\left(s-\bar{m}^{2}\right)^{2}}{331776 \pi^{8}}+\frac{y\left(s-\bar{m}^{2}\right)^{4}}{663552 \pi^{8}}\right\} \\
& +\langle\bar{q} q\rangle^{2} \int_{4 m_{c}^{2}}^{s_{0}} d s \int_{y_{i}}^{y_{f}} d y \int_{z_{i}}^{1-y} d z e^{-\frac{s}{T^{2}}}\left\{\frac{z(y+z-1)^{4}}{y}\right\}\left\{\frac{s y\left(s-\bar{m}^{2}\right)^{3}}{165888 \pi^{8}}-\frac{\left(s-\bar{m}^{2}\right)^{4}}{995328 \pi^{8}}-\frac{s\left(s-\bar{m}^{2}\right)^{3}}{497664 \pi^{8}}\right\} \\
& +\langle\bar{q} q\rangle^{2} \int_{4 m_{c}^{2}}^{s_{0}} d s \int_{y_{i}}^{y_{f}} d y \int_{z_{i}}^{1-y} d z e^{-\frac{s}{T^{2}}}\left\{\frac{z(y+z-1)^{4}}{y}\right\}\left\{-\frac{s^{2} y\left(s-\bar{m}^{2}\right)^{2}}{331776 \pi^{8}}-\frac{y\left(s-\bar{m}^{2}\right)^{4}}{663552 \pi^{8}}\right\} \\
& +\langle\bar{q} q\rangle^{2} \int_{4 m_{c}^{2}}^{s_{0}} d s \int_{y_{i}}^{y_{f}} d y \int_{z_{i}}^{1-y} d z e^{-\frac{s}{T^{2}}}\left\{\frac{z(y+z-1)^{4}}{y}\right\}\left\{-\frac{s y\left(s-\bar{m}^{2}\right)^{3}}{165888 \pi^{8}}+\frac{\left(s-\bar{m}^{2}\right)^{4}}{3981312 \pi^{8}}+\frac{s\left(s-\bar{m}^{2}\right)^{3}}{1990656 \pi^{8}}\right\},
\end{aligned}
$$

$$
\begin{aligned}
\mathbf{S}_{B}(8) & =m_{c}^{2}\langle\bar{q} q\rangle\left\langle\bar{q} g_{s} \sigma G q\right\rangle \int_{4 m_{c}^{2}}^{s_{0}} d s \int_{y_{i}}^{y_{f}} d y \int_{z_{i}}^{1-y} d z e^{-\frac{s}{T^{2}}}\{-y-z+1\}\left\{-\frac{\left(s-\bar{m}^{2}\right)^{2}}{512 \pi^{6}}\right\} \\
& +\langle\bar{q} q\rangle\left\langle\bar{q} g_{s} \sigma G q\right\rangle \int_{4 m_{c}^{2}}^{s_{0}} d s \int_{y_{i}}^{y_{f}} d y \int_{z_{i}}^{1-y} d z e^{-\frac{s}{T^{2}}}\{-y z(y+z-1)\}\left\{-\frac{\left(s-\bar{m}^{2}\right)^{3}}{768 \pi^{6}}-\frac{s\left(s-\bar{m}^{2}\right)^{2}}{512 \pi^{6}}\right\}
\end{aligned}
$$




$$
\begin{aligned}
\mathbf{S}_{B}(10)_{1} & =m_{c}^{2}\left\langle\bar{q} g_{s} \sigma G q\right\rangle^{2} \int_{4 m_{c}^{2}}^{s_{0}} d s \int_{y_{i}}^{y_{f}} d y \int_{z_{i}}^{1-y} d z e^{-\frac{s}{T^{2}}}\left\{-\frac{\bar{m}^{2}-s}{2048 \pi^{6}}\right\} \\
& +\left\langle\bar{q} g_{s} \sigma G q\right\rangle^{2} \int_{4 m_{c}^{2}}^{s_{0}} d s \int_{y_{i}}^{y_{f}} d y \int_{z_{i}}^{1-y} d z e^{-\frac{s}{T^{2}}}\{y z\}\left\{\frac{\left(s-\bar{m}^{2}\right)^{2}}{2048 \pi^{6}}+\frac{s\left(s-\bar{m}^{2}\right.}{2048 \pi^{6}}\right\} \\
& +\left\langle\bar{q} g_{s} \sigma G q\right\rangle^{2} \int_{4 m_{c}^{2}}^{s_{0}} d s \int_{y_{i}}^{y_{f}} d y \int_{z_{i}}^{1-y} d z e^{-\frac{s}{T^{2}}}\{y z\}\left\{\frac{\left(s-\bar{m}^{2}\right)^{2}}{98304 \pi^{6}}+\frac{s\left(s-\bar{m}^{2}\right)}{98304 \pi^{6}}\right\} \\
& +m_{c}^{2}\left\langle\bar{q} g_{s} \sigma G q\right\rangle^{2} \int_{4 m_{c}^{2}}^{s_{0}} d s \int_{y_{i}}^{y_{f}} d y \int_{z_{i}}^{1-y} d z e^{-\frac{s}{T^{2}}}\left\{-\frac{\bar{m}^{2}-s}{98304 \pi^{6}}\right\}
\end{aligned}
$$

$$
\begin{aligned}
\mathbf{S}_{B}(10)_{2}= & m_{c}^{2}\langle\bar{q} q\rangle^{2}\left\langle g_{s}^{2} G G\right\rangle \int_{4 m_{c}^{2}}^{s_{0}} d s \int_{y_{i}}^{y_{f}} d y \int_{z_{i}}^{1-y} d z e^{-\frac{s}{T^{2}}}\left\{-\frac{(y+z-1)^{2}}{y^{2}}\right\}\left\{\frac{s y}{9216 \pi^{6}}-\frac{(y-1)\left(\bar{m}^{2}-s\right)}{4608 \pi^{6}}\right\} \\
& +m_{c}^{2}\langle\bar{q} q\rangle^{2}\left\langle g_{s}^{2} G G\right\rangle \int_{4 m_{c}^{2}}^{s_{0}} d s \int_{y_{i}}^{y_{f}} d y \int_{z_{i}}^{1-y} d z e^{-\frac{s}{T^{2}}}\left\{\frac{z(y+z-1)^{2}}{y^{2}}\right\}\left\{\frac{\bar{m}^{2}-s}{4608 \pi^{6}}-\frac{s}{9216 \pi^{6}}\right\} \\
& +m_{c}^{2}\langle\bar{q} q\rangle^{2}\left\langle g_{s}^{2} G G\right\rangle \int_{4 m_{c}^{2}}^{s_{0}} d s \int_{y_{i}}^{y_{f}} d y \int_{z_{i}}^{1-y} d z e^{-\frac{s}{T^{2}}}\left\{-\frac{\bar{m}^{2}-s}{6144 \pi^{6}}\right\} \\
& +\langle\bar{q} q\rangle^{2}\left\langle g_{s}^{2} G G\right\rangle \int_{4 m_{c}^{2}}^{s_{0}} d s \int_{y_{i}}^{y_{f}} d y \int_{z_{i}}^{1-y} d z e^{-\frac{s}{T^{2}}}\{y z\}\left\{\frac{\left(s-\bar{m}^{2}\right)^{2}}{6144 \pi^{6}}+\frac{s\left(s-\bar{m}^{2}\right.}{6144 \pi^{6}}\right\} \\
& +m_{c}^{2}\langle\bar{q} q\rangle^{2}\left\langle g_{s}^{2} G G\right\rangle \int_{4 m_{c}^{2}}^{s_{0}} d s \int_{y_{i}}^{y_{f}} d y \int_{z_{i}}^{1-y} d z e^{-\frac{s}{T^{2}}}\left\{-\frac{\bar{m}^{2}-s}{9216 \pi^{6}}\right\} \\
& +\langle\bar{q} q\rangle^{2}\left\langle g_{s}^{2} G G\right\rangle \int_{4 m_{c}^{2}}^{s_{0}} d s \int_{y_{i}}^{y_{f}} d y \int_{z_{i}}^{1-y} d z e^{-\frac{s}{T^{2}}}\{y z\}\left\{\frac{\left(s-\bar{m}^{2}\right)^{2}}{9216 \pi^{6}}+\frac{s\left(s-\bar{m}^{2}\right)}{9216 \pi^{6}}\right\} \\
& +m_{c}^{2}\left\langle g_{s}^{2} G G\right\rangle\langle\bar{q} q\rangle^{2} \int_{4 m_{c}^{2}}^{s_{0}} d s \int_{y_{i}}^{y_{f}} d y \int_{z_{i}}^{1-y} d z e^{-\frac{s}{T^{2}}}\left\{-\frac{(y+z-1)^{2}}{y^{2}}\right\}\left\{\frac{(1-y)\left(\bar{m}^{2}-s\right)}{248832 \pi^{8}}+\frac{s y}{497664 \pi^{8}}\right\} \\
& +m_{c}^{2}\left\langle g_{s}^{2} G G\right\rangle\langle\bar{q} q\rangle^{2} \int_{4 m_{c}^{2}}^{s_{0}} d s \int_{y_{i}}^{y_{f}} d y \int_{z_{i}}^{1-y} d z e^{-\frac{s}{T^{2}}}\left\{\frac{z(y+z-1)^{2}}{y^{2}}\right\}\left\{\frac{\bar{m}^{2}-s}{248832 \pi^{8}}-\frac{s}{497664 \pi^{8}}\right\}
\end{aligned}
$$

$$
\begin{aligned}
\mathbf{S}_{B}(12)_{1} & =m_{c}^{2}\langle\bar{q} q\rangle^{4} \int_{4 m_{c}^{2}}^{s_{0}} d s \int_{y_{i}}^{y_{f}} d y \int_{z_{i}}^{1-y} d z e^{-\frac{s}{T^{2}}}\left\{\frac{y+z-1}{y}\right\}\left\{\frac{1}{1296 \pi^{4}}\right\} \\
& +m_{c}^{2}\langle\bar{q} q\rangle^{4} \int_{4 m_{c}^{2}}^{s_{0}} d s \int_{y_{i}}^{y_{f}} d y \int_{z_{i}}^{1-y} d z e^{-\frac{s}{T^{2}}}\left\{-\frac{z(y+z-1)}{y^{2}}\right\}\left\{-\frac{1}{1296 \pi^{4}}\right\} \\
& +\langle\bar{q} q\rangle^{4} \int_{4 m_{c}^{2}}^{s_{0}} d s \int_{y_{i}}^{y_{f}} d y \int_{z_{i}}^{1-y} d z e^{-\frac{s}{T^{2}}}\left\{-\frac{z(y+z-1)}{y}\right\}\left\{-\frac{\bar{m}^{2} y}{648 \pi^{4}}+\frac{\bar{m}^{2}}{972 \pi^{4}}+\frac{s y}{324 \pi^{4}}-\frac{s}{648 \pi^{4}}\right\} \\
& +\langle\bar{q} q\rangle^{4} \int_{4 m_{c}^{2}}^{s_{0}} d s \int_{y_{i}}^{y_{f}} d y \int_{z_{i}}^{1-y} d z e^{-\frac{s}{T^{2}}}\left\{-\frac{z(y+z-1)}{y}\right\}\left\{\frac{\bar{m}^{2} y}{648 \pi^{4}}-\frac{\bar{m}^{2}}{3888 \pi^{4}}-\frac{s y}{324 \pi^{4}}+\frac{s}{2592 \pi^{4}}\right\},
\end{aligned}
$$




$$
\begin{aligned}
\mathbf{S}_{B}(12)_{2} \quad & =m_{c}^{2}\left\langle\bar{q} g_{s} \sigma G q\right\rangle\langle\bar{q} q\rangle\left\langle g_{s}^{2} G G\right\rangle \int_{4 m_{c}^{2}}^{s_{0}} d s \int_{y_{i}}^{y_{f}} d y \int_{z_{i}}^{1-y} d z e^{-\frac{s}{T^{2}}}\left\{\frac{y+z-1}{y^{2}}\right\}\left\{\frac{1-y}{4608 \pi^{6}}\right\} \\
& +m_{c}^{2}\left\langle\bar{q} g_{s} \sigma G q\right\rangle\langle\bar{q} q\rangle\left\langle g_{s}^{2} G G\right\rangle \int_{4 m_{c}^{2}}^{s_{0}} d s \int_{y_{i}}^{y_{f}} d y \int_{z_{i}}^{1-y} d z e^{-\frac{s}{T^{2}}}\left\{-\frac{z(y+z-1)}{y^{2}}\right\}\left\{\frac{1}{4608 \pi^{6}}\right\} .
\end{aligned}
$$

Type C for $J_{2}$

$$
\begin{aligned}
\mathbf{S}_{C}(14) & =m_{c}^{2}\langle\bar{q} q\rangle^{3}\left\langle\bar{q} g_{s} \sigma G q\right\rangle \int_{y_{i}}^{y_{f}} d y e^{-\frac{\tilde{m}^{2}}{T^{2}}}\left\{-\frac{1}{144 \pi^{2}}-\frac{\tilde{m}^{2}}{144 \pi^{2} T^{2}}\right\} \\
& +\langle\bar{q} q\rangle^{3}\left\langle\bar{q} g_{s} \sigma G q\right\rangle \int_{y_{i}}^{y_{f}} d y e^{-\frac{\tilde{m}^{2}}{T^{2}}}\{(y-1) y\}\left\{\frac{\tilde{m}^{2}}{36 \pi^{2}}+\frac{\tilde{m}^{4}}{144 \pi^{2} T^{2}}\right\} \\
& +m_{c}^{2}\langle\bar{q} q\rangle^{3}\left\langle\bar{q} g_{s} \sigma G q\right\rangle \int_{y_{i}}^{y_{f}} d y e^{-\frac{\tilde{m}^{2}}{T^{2}}}\left\{-\frac{1}{7776 \pi^{4}}-\frac{\tilde{m}^{2}}{7776 \pi^{4} T^{2}}\right\} \\
& +\langle\bar{q} q\rangle^{3}\left\langle\bar{q} g_{s} \sigma G q\right\rangle \int_{y_{i}}^{y_{f}} d y e^{-\frac{\tilde{m}^{2}}{T^{2}}}\{(y-1) y\}\left\{\frac{\tilde{m}^{2}}{1944 \pi^{4}}+\frac{\tilde{m}^{4}}{7776 \pi^{4} T^{2}}\right\},
\end{aligned}
$$

$$
\begin{aligned}
\mathbf{S}_{C}(16)_{1} & =\langle\bar{q} q\rangle^{2}\left\langle\bar{q} g_{s} \sigma G q\right\rangle^{2} \int_{y_{i}}^{y_{f}} d y e^{-\frac{\tilde{m}^{2}}{T^{2}}}\{(y-1) y\}\left\{-\frac{1}{64 \pi^{2}}-\frac{\tilde{m}^{6}}{384 \pi^{2} T^{6}}-\frac{\tilde{m}^{4}}{128 \pi^{2} T^{4}}-\frac{\tilde{m}^{2}}{64 \pi^{2} T^{2}}\right\} \\
& +m_{c}^{2}\langle\bar{q} q\rangle^{2}\left\langle\bar{q} g_{s} \sigma G q\right\rangle^{2} \int_{y_{i}}^{y_{f}} d y e^{-\frac{\tilde{m}^{2}}{T^{2}}}\left\{\frac{\tilde{m}^{4}}{384 \pi^{2} T^{6}}\right\} \\
& +m_{c}^{2}\langle\bar{q} q\rangle^{2}\left\langle\bar{q} g_{s} \sigma G q\right\rangle^{2} \int_{y_{i}}^{y_{f}} d y e^{-\frac{\tilde{m}^{2}}{T^{2}}}\left\{\frac{\tilde{m}^{4}}{62208 \pi^{4} T^{6}}\right\} \\
& +\langle\bar{q} q\rangle^{2}\left\langle\bar{q} g_{s} \sigma G q\right\rangle^{2} \int_{y_{i}}^{y_{f}} d y e^{-\frac{\tilde{m}^{2}}{T^{2}}}\{(y-1) y\}\left\{-\frac{1}{10368 \pi^{4}}-\frac{\tilde{m}^{6}}{62208 \pi^{4} T^{6}}-\frac{\tilde{m}^{4}}{20736 \pi^{4} T^{4}}-\frac{\tilde{m}^{2}}{10368 \pi^{4} T^{2}}\right\},
\end{aligned}
$$

$$
\begin{aligned}
\mathbf{S}_{C}(16)_{2}= & m_{c}^{2}\left\langle g_{s}^{2} G G\right\rangle\langle\bar{q} q\rangle^{4} \int_{y_{i}}^{y_{f}} d y e^{-\frac{\tilde{m}^{2}}{T^{2}}}\left\{\frac{1}{y^{2}}\right\}\left\{\frac{1}{1728 \pi^{2} T^{2}}\right\} \\
& +m_{c}^{4}\left\langle g_{s}^{2} G G\right\rangle\langle\bar{q} q\rangle^{4} \int_{y_{i}}^{y_{f}} d y e^{-\frac{\tilde{m}^{2}}{T^{2}}}\left\{\frac{1}{y^{3}}\right\}\left\{-\frac{1}{5184 \pi^{2} T^{4}}\right\} \\
& +m_{c}^{2}\left\langle g_{s}^{2} G G\right\rangle\langle\bar{q} q\rangle^{4} \int_{y_{i}}^{y_{f}} d y e^{-\frac{\tilde{m}^{2}}{T^{2}}}\left\{\frac{y-1}{y^{2}}\right\}\left\{\frac{\tilde{m}^{2}}{5184 \pi^{2} T^{4}}\right\} \\
& +m_{c}^{2}\langle\bar{q} q\rangle^{4}\left\langle g_{s}^{2} G G\right\rangle \int_{y_{i}}^{y_{f}} d y e^{-\frac{\tilde{m}^{2}}{T^{2}}}\left\{\frac{\tilde{m}^{4}}{5184 \pi^{2} T^{6}}\right\} \\
& +\langle\bar{q} q\rangle^{4}\left\langle g_{s}^{2} G G\right\rangle \int_{y_{i}}^{y_{f}} d y e^{-\frac{\tilde{m}^{2}}{T^{2}}}\{(y-1) y\}\left\{-\frac{1}{864 \pi^{2}}-\frac{\tilde{m}^{6}}{5184 \pi^{2} T^{6}}-\frac{\tilde{m}^{4}}{1728 \pi^{2} T^{4}}-\frac{\tilde{m}^{2}}{864 \pi^{2} T^{2}}\right\} .
\end{aligned}
$$


Type D for $J_{2}$

$$
\begin{gathered}
\mathbf{S}_{D}(12)_{1}=m_{c}^{2}\left\langle\bar{q} g_{s} \sigma G q\right\rangle\langle\bar{q} q\rangle\left\langle g_{s}^{2} G G\right\rangle \int_{y_{i}}^{y_{f}} d y \int_{z_{i}}^{1-y} d z e^{-\frac{\bar{m}^{2}}{T^{2}}}\left\{\frac{y+z-1}{y^{2}}\right\}\left\{-\frac{\bar{m}^{2} y}{9216 \pi^{6}}\right\} \\
+m_{c}^{2}\left\langle\bar{q} g_{s} \sigma G q\right\rangle\langle\bar{q} q\rangle\left\langle g_{s}^{2} G G\right\rangle \int_{y_{i}}^{y_{f}} d y \int_{z_{i}}^{1-y} d z e^{-\frac{\bar{m}^{2}}{T^{2}}}\left\{-\frac{z(y+z-1)}{y^{2}}\right\}\left\{\frac{\bar{m}^{2}}{9216 \pi^{6}}\right\} \\
\mathbf{S}_{D}(12)_{2}=m_{c}^{2}\langle\bar{q} q\rangle^{4} \int_{y_{i}}^{y_{f}} d y \int_{z_{i}}^{1-y} d z e^{-\frac{\bar{m}^{2}}{T^{2}}}\left\{\frac{y+z-1}{y}\right\}\left\{\frac{\bar{m}^{2}}{2592 \pi^{4}}\right\} \\
+m_{c}^{2}\langle\bar{q} q\rangle^{4} \int_{y_{i}}^{y_{f}} d y \int_{z_{i}}^{1-y} d z e^{-\frac{\bar{m}^{2}}{T^{2}}}\left\{-\frac{z(y+z-1)}{y^{2}}\right\}\left\{-\frac{\bar{m}^{2}}{2592 \pi^{4}}\right\} \\
+\langle\bar{q} q\rangle^{4} \int_{y_{i}}^{y_{f}} d y \int_{z_{i}}^{1-y} d z e^{-\frac{\bar{m}^{2}}{T^{2}}}\left\{-\frac{z(y+z-1)}{y}\right\}\left\{\frac{\bar{m}^{4} y}{3888 \pi^{4}}\right\} \\
+\langle\bar{q} q\rangle^{4} \int_{y_{i}}^{y_{f}} d y \int_{z_{i}}^{1-y} d z e^{-\frac{\bar{m}^{2}}{T^{2}}}\left\{-\frac{z(y+z-1)}{y}\right\}\left\{-\frac{\bar{m}^{4} y}{3888 \pi^{4}}\right\} .
\end{gathered}
$$


Type A for $J_{3}$

$$
\begin{aligned}
\mathbf{S}_{A}(12) & =\langle\bar{q} q\rangle^{4} \int_{4 m_{c}^{2}}^{s_{0}} d s \int_{y_{i}}^{y_{f}} d y e^{-\frac{s}{T^{2}}}\{(y-1) y\}\left\{\frac{\tilde{m}^{2}-s}{216 \pi^{2}}-\frac{s}{432 \pi^{2}}\right\} \\
& +\langle\bar{q} q\rangle^{4} \int_{4 m_{c}^{2}}^{s_{0}} d s \int_{y_{i}}^{y_{f}} d y e^{-\frac{s}{T^{2}}}\{(y-1) y\}\left\{\frac{\tilde{m}^{2}-s}{432 \pi^{2}}-\frac{s}{216 \pi^{2}}\right\} \\
& -m_{c}^{2}\langle\bar{q} q\rangle^{4} \int_{4 m_{c}^{2}}^{s_{0}} d s \int_{y_{i}}^{y_{f}} d y e^{-\frac{s}{T^{2}}}\left\{\frac{1}{144 \pi^{2}}\right\} \\
& +\langle\bar{q} q\rangle^{4} \int_{4 m_{c}^{2}}^{s_{0}} d s \int_{y_{i}}^{y_{f}} d y e^{-\frac{s}{T^{2}}}\{(y-1) y\}\left\{\frac{\tilde{m}^{2}-s}{11664 \pi^{4}}-\frac{s}{5832 \pi^{4}}\right\} \\
& -m_{c}^{2}\langle\bar{q} q\rangle^{4} \int_{4 m_{c}^{2}}^{s_{0}} d s \int_{y_{i}}^{y_{f}} d y e^{-\frac{s}{T^{2}}}\left\{\frac{1}{3888 \pi^{4}}\right\} \\
& +\langle\bar{q} q\rangle^{4} \int_{4 m_{c}^{2}}^{s_{0}} d s \int_{y_{i}}^{y_{f}} d y e^{-\frac{s}{T^{2}}}\{(y-1) y\}\left\{\frac{\tilde{m}^{2}-s}{5832 \pi^{4}}-\frac{s}{11664 \pi^{4}}\right\},
\end{aligned}
$$

$$
\begin{aligned}
\mathbf{S}_{A}(14) & =\left\langle\bar{q} g_{s} \sigma G q\right\rangle\langle\bar{q} q\rangle^{3} \int_{4 m_{c}^{2}}^{s_{0}} d s \int_{y_{i}}^{y_{f}} d y e^{-\frac{s}{T^{2}}}\{(y-1) y\}\left\{\frac{1}{72 \pi^{2}}\right\} \\
& +\left\langle\bar{q} g_{s} \sigma G q\right\rangle\langle\bar{q} q\rangle^{3} \int_{4 m_{c}^{2}}^{s_{0}} d s \int_{y_{i}}^{y_{f}} d y e^{-\frac{s}{T^{2}}}\{(y-1) y\}\left\{\frac{1}{144 \pi^{2}}\right\} \\
& +\langle\bar{q} q\rangle^{3}\left\langle\bar{q} g_{s} \sigma G q\right\rangle \int_{4 m_{c}^{2}}^{s_{0}} d s \int_{y_{i}}^{y_{f}} d y e^{-\frac{s}{T^{2}}}\{(y-1) y\}\left\{\frac{1}{7776 \pi^{4}}\right\} \\
& +\langle\bar{q} q\rangle^{3}\left\langle\bar{q} g_{s} \sigma G q\right\rangle \int_{4 m_{c}^{2}}^{s_{0}} d s \int_{y_{i}}^{y_{f}} d y e^{-\frac{s}{T^{2}}}\{(y-1) y\}\left\{\frac{1}{3888 \pi^{4}}\right\} .
\end{aligned}
$$

Type B for $J_{3}$

$$
\begin{aligned}
\mathbf{S}_{B}(0) & =\int_{4 m_{c}^{2}}^{s_{0}} d s \int_{y_{i}}^{y_{f}} d y \int_{z_{i}}^{1-y} d z e^{-\frac{s}{T^{2}}}\left\{-y z(y+z-1)^{5}\right\}\left\{\frac{\left(s-\bar{m}^{2}\right)^{7}}{412876800 \pi^{10}}+\frac{s\left(s-\bar{m}^{2}\right)^{6}}{117964800 \pi^{10}}\right\} \\
& +\int_{4 m_{c}^{2}}^{s_{0}} d s \int_{y_{i}}^{y_{f}} d y \int_{z_{i}}^{1-y} d z e^{-\frac{s}{T^{2}}}\left\{-y z(y+z-1)^{5}\right\}\left\{\frac{\left(s-\bar{m}^{2}\right)^{7}}{825753600 \pi^{10}}+\frac{s\left(s-\bar{m}^{2}\right)^{6}}{58982400 \pi^{10}}\right\} \\
& -m_{c}^{2} \int_{4 m_{c}^{2}}^{s_{0}} d s \int_{y_{i}}^{y_{f}} d y \int_{z_{i}}^{1-y} d z e^{-\frac{s}{T^{2}}}\left\{-(y+z-1)^{5}\right\}\left\{\frac{\left(s-\bar{m}^{2}\right)^{6}}{39321600 \pi^{10}}\right\}
\end{aligned}
$$




$$
\begin{aligned}
\mathbf{S}_{B}(4) & =m_{c}^{2}\left\langle g_{s}^{2} G G\right\rangle \int_{4 m_{c}^{2}}^{s_{0}} d s \int_{y_{i}}^{y_{f}} d y \int_{z_{i}}^{1-y} d z e^{-\frac{s}{T^{2}}}\left\{-\frac{z(y+z-1)^{5}}{y^{2}}\right\}\left\{-\frac{\left(s-\bar{m}^{2}\right)^{4}}{141557760 \pi^{10}}-\frac{s\left(s-\bar{m}^{2}\right)^{3}}{17694720 \pi^{10}}\right\} \\
& -m_{c}^{2}\left\langle g_{s}^{2} G G\right\rangle \int_{4 m_{c}^{2}}^{s_{0}} d s \int_{y_{i}}^{y_{f}} d y \int_{z_{i}}^{1-y} d z e^{-\frac{s}{T^{2}}}\left\{\frac{(y+z-1)^{5}}{y^{2}}\right\}\left\{\frac{s y\left(s-\bar{m}^{2}\right)^{3}}{11796480 \pi^{10}}-\frac{(1-y)\left(s-\bar{m}^{2}\right)^{4}}{23592960 \pi^{10}}\right\} \\
& +m_{c}^{2}\left\langle g_{s}^{2} G G\right\rangle \int_{4 m_{c}^{2}}^{s_{0}} d s \int_{y_{i}}^{y_{f}} d y \int_{z_{i}}^{1-y} d z e^{-\frac{s}{T^{2}}}\left\{-\frac{z(y+z-1)^{5}}{y^{2}}\right\}\left\{-\frac{\left(s-\bar{m}^{2}\right)^{4}}{70778880 \pi^{10}}-\frac{s\left(s-\bar{m}^{2}\right)^{3}}{35389440 \pi^{10}}\right\} \\
& +\left\langle g_{s}^{2} G G\right\rangle \int_{4 m_{c}^{2}}^{s_{0}} d s \int_{y_{i}}^{y_{f}} d y \int_{z_{i}}^{1-y} d z e^{-\frac{s}{T^{2}}}\left\{-y z(y+z-1)^{3}\right\}\left\{\frac{\left(s-\bar{m}^{2}\right)^{5}}{23592960 \pi^{10}}+\frac{s\left(s-\bar{m}^{2}\right)^{4}}{2359296 \pi^{10}}\right\} \\
& -m_{c}^{2}\left\langle g_{s}^{2} G G\right\rangle \int_{4 m_{c}^{2}}^{s_{0}} d s \int_{y_{i}}^{y_{f}} d y \int_{z_{i}}^{1-y} d z e^{-\frac{s}{T^{2}}}\left\{-(y+z-1)^{3}\right\}\left\{\frac{\left(s-\bar{m}^{2}\right)^{4}}{1572864 \pi^{10}}\right\} \\
& +\left\langle g_{s}^{2} G G\right\rangle \int_{4 m_{c}^{2}}^{s_{0}} d s \int_{y_{i}}^{y_{f}} d y \int_{z_{i}}^{1-y} d z e^{-\frac{s}{T^{2}}}\left\{-y z(y+z-1)^{3}\right\}\left\{\frac{\left(s-\bar{m}^{2}\right)^{5}}{11796480 \pi^{10}}+\frac{s\left(s-\bar{m}^{2}\right)^{4}}{4718592 \pi^{10}}\right\}
\end{aligned}
$$

$$
\begin{aligned}
\mathbf{S}_{B}(6) & =\langle\bar{q} q\rangle^{2} \int_{4 m_{c}^{2}}^{s_{0}} d s \int_{y_{i}}^{y_{f}} d y \int_{z_{i}}^{1-y} d z e^{-\frac{s}{T^{2}}}\left\{y z(y+z-1)^{2}\right\}\left\{\frac{\left(s-\bar{m}^{2}\right)^{4}}{9216 \pi^{6}}+\frac{s\left(s-\bar{m}^{2}\right)^{3}}{4608 \pi^{6}}\right\} \\
& +\langle\bar{q} q\rangle^{2} \int_{4 m_{c}^{2}}^{s_{0}} d s \int_{y_{i}}^{y_{f}} d y \int_{z_{i}}^{1-y} d z e^{-\frac{s}{T^{2}}}\left\{y z(y+z-1)^{2}\right\}\left\{\frac{\left(s-\bar{m}^{2}\right)^{4}}{18432 \pi^{6}}+\frac{s\left(s-\bar{m}^{2}\right)^{3}}{2304 \pi^{6}}\right\} \\
& -m_{c}^{2}\langle\bar{q} q\rangle^{2} \int_{4 m_{c}^{2}}^{s_{0}} d s \int_{y_{i}}^{y_{f}} d y \int_{z_{i}}^{1-y} d z e^{-\frac{s}{T^{2}}}\left\{(y+z-1)^{2}\right\}\left\{\frac{\left(s-\bar{m}^{2}\right)^{3}}{1536 \pi^{6}}\right\} \\
& +\langle\bar{q} q\rangle^{2} \int_{4 m_{c}^{2}}^{s_{0}} d s \int_{y_{i}}^{y_{f}} d y \int_{z_{i}}^{1-y} d z e^{-\frac{s}{T^{2}}}\left\{y z(y+z-1)^{2}\right\}\left\{\frac{\left(s-\bar{m}^{2}\right)^{4}}{995328 \pi^{8}}+\frac{s\left(s-\bar{m}^{2}\right)^{3}}{124416 \pi^{8}}\right\} \\
& -m_{c}^{2}\langle\bar{q} q\rangle^{2} \int_{4 m_{c}^{2}}^{s_{0}} d s \int_{y_{i}}^{y_{f}} d y \int_{z_{i}}^{1-y} d z e^{-\frac{s}{T^{2}}}\left\{(y+z-1)^{2}\right\}\left\{\frac{\left(s-\bar{m}^{2}\right)^{3}}{82944 \pi^{8}}\right\} \\
& +\langle\bar{q} q\rangle^{2} \int_{4 m_{c}^{2}}^{s_{0}} d s \int_{y_{i}}^{y_{f}} d y \int_{z_{i}}^{1-y} d z e^{-\frac{s}{T^{2}}}\left\{y z(y+z-1)^{2}\right\}\left\{\frac{\left(s-\bar{m}^{2}\right)^{4}}{497664 \pi^{8}}+\frac{s\left(s-\bar{m}^{2}\right)^{3}}{248832 \pi^{8}}\right\}
\end{aligned}
$$

$$
\begin{aligned}
\mathbf{S}_{B}(8) & =\left\langle\bar{q} g_{s} \sigma G q\right\rangle\langle\bar{q} q\rangle \int_{4 m_{c}^{2}}^{s_{0}} d s \int_{y_{i}}^{y_{f}} d y \int_{z_{i}}^{1-y} d z e^{-\frac{s}{T^{2}}}\{-y z(y+z-1)\}\left\{-\frac{\left(s-\bar{m}^{2}\right)^{3}}{2304 \pi^{6}}-\frac{s\left(s-\bar{m}^{2}\right)^{2}}{1536 \pi^{6}}\right\} \\
& +\left\langle\bar{q} g_{s} \sigma G q\right\rangle\langle\bar{q} q\rangle \int_{4 m_{c}^{2}}^{s_{0}} d s \int_{y_{i}}^{y_{f}} d y \int_{z_{i}}^{1-y} d z e^{-\frac{s}{T^{2}}}\{-y z(y+z-1)\}\left\{-\frac{\left(s-\bar{m}^{2}\right)^{3}}{4608 \pi^{6}}-\frac{s\left(s-\bar{m}^{2}\right)^{2}}{768 \pi^{6}}\right\} \\
& -m_{c}^{2}\left\langle\bar{q} g_{s} \sigma G q\right\rangle\langle\bar{q} q\rangle \int_{4 m_{c}^{2}}^{s_{0}} d s \int_{y_{i}}^{y_{f}} d y \int_{z_{i}}^{1-y} d z e^{-\frac{s}{T^{2}}}\{-y-z+1\}\left\{-\frac{\left(s-\bar{m}^{2}\right)^{2}}{512 \pi^{6}}\right\}
\end{aligned}
$$




$$
\begin{aligned}
\mathbf{S}_{B}(10)_{1} & =m_{c}^{2}\langle\bar{q} q\rangle^{2}\left\langle g_{s}^{2} G G\right\rangle \int_{4 m_{c}^{2}}^{s_{0}} d s \int_{y_{i}}^{y_{f}} d y \int_{z_{i}}^{1-y} d z e^{-\frac{s}{T^{2}}}\left\{\frac{z(y+z-1)^{2}}{y^{2}}\right\}\left\{\frac{\bar{m}^{2}-s}{27648 \pi^{6}}-\frac{s}{13824 \pi^{6}}\right\} \\
& -m_{c}^{2}\left\langle g_{s}^{2} G G\right\rangle\langle\bar{q} q\rangle^{2} \int_{4 m_{c}^{2}}^{s_{0}} d s \int_{y_{i}}^{y_{f}} d y \int_{z_{i}}^{1-y} d z e^{-\frac{s}{T^{2}}}\left\{-\frac{(y+z-1)^{2}}{y^{2}}\right\}\left\{\frac{(1-y)\left(\bar{m}^{2}-s\right)}{4608 \pi^{6}}+\frac{s y}{9216 \pi^{6}}\right\} \\
& +m_{c}^{2}\left\langle g_{s}^{2} G G\right\rangle\langle\bar{q} q\rangle^{2} \int_{4 m_{c}^{2}}^{s_{0}} d s \int_{y_{i}}^{y_{f}} d y \int_{z_{i}}^{1-y} d z e^{-\frac{s}{T^{2}}}\left\{\frac{z(y+z-1)^{2}}{y^{2}}\right\}\left\{\frac{\bar{m}^{2}-s}{13824 \pi^{6}}-\frac{s}{27648 \pi^{6}}\right\} \\
& +\langle\bar{q} q\rangle^{2}\left\langle g_{s}^{2} G G\right\rangle \int_{4 m_{c}^{2}}^{s_{0}} d s \int_{y_{i}}^{y_{f}} d y \int_{z_{i}}^{1-y} d z e^{-\frac{s}{T^{2}}}\{y z\}\left\{\frac{\left(s-\bar{m}^{2}\right)^{2}}{36864 \pi^{6}}+\frac{s\left(s-\bar{m}^{2}\right)}{9216 \pi^{6}}\right\} \\
& -m_{c}^{2}\left\langle g_{s}^{2} G G\right\rangle\langle\bar{q} q\rangle^{2} \int_{4 m_{c}^{2}}^{s_{0}} d s \int_{y_{i}}^{y_{f}} d y \int_{z_{i}}^{1-y} d z e^{-\frac{s}{T^{2}}}\left\{-\frac{\bar{m}^{2}-s}{6144 \pi^{6}}\right\} \\
& +\left\langle g_{s}^{2} G G\right\rangle\langle\bar{q} q\rangle^{2} \int_{4 m_{c}^{2}}^{s_{0}} d s \int_{y_{i}}^{y_{f}} d y \int_{z_{i}}^{1-y} d z e^{-\frac{s}{T^{2}}}\{y z\}\left\{\frac{\left(s-\bar{m}^{2}\right)^{2}}{18432 \pi^{6}}+\frac{s\left(s-\bar{m}^{2}\right)}{18432 \pi^{6}}\right\} \\
& +\langle\bar{q} q\rangle^{2}\left\langle g_{s}^{2} G G\right\rangle \int_{4 m_{c}^{2}}^{s_{0}} d s \int_{y_{i}}^{y_{f}} d y \int_{z_{i}}^{1-y} d z e^{-\frac{s}{T^{2}}}\{y z\}\left\{\frac{\left(s-\bar{m}^{2}\right)^{2}}{55296 \pi^{6}}+\frac{s\left(s-\bar{m}^{2}\right)}{13824 \pi^{6}}\right\} \\
& -m_{c}^{2}\langle\bar{q} q\rangle^{2}\left\langle g_{s}^{2} G G\right\rangle \int_{4 m_{c}^{2}}^{s_{0}} d s \int_{y_{i}}^{y_{f}} d y \int_{z_{i}}^{1-y} d z e^{-\frac{s}{T^{2}}}\left\{-\frac{\bar{m}^{2}-s}{9216 \pi^{6}}\right\} \\
& +\langle\bar{q} q\rangle^{2}\left\langle g_{s}^{2} G G\right\rangle \int_{4 m_{c}^{2}}^{s_{0}} d s \int_{y_{i}}^{y_{f}} d y \int_{z_{i}}^{1-y} d z e^{-\frac{s}{T^{2}}}\{y z\}\left\{\frac{\left(s-\bar{m}^{2}\right)^{2}}{27648 \pi^{6}}+\frac{s\left(s-\bar{m}^{2}\right)}{27648 \pi^{6}}\right\}
\end{aligned}
$$

$$
\begin{aligned}
\mathbf{S}_{B}(10)_{2} & +\left\langle\bar{q} g_{s} \sigma G q\right\rangle^{2} \int_{4 m_{c}^{2}}^{s_{0}} d s \int_{y_{i}}^{y_{f}} d y \int_{z_{i}}^{1-y} d z e^{-\frac{s}{T^{2}}}\left\{(y z\}\left\{\frac{\left(s-\bar{m}^{2}\right)^{2}}{6144 \pi^{6}}+\frac{s\left(s-\bar{m}^{2}\right)}{6144 \pi^{6}}\right\}\right. \\
& +\left\langle\bar{q} g_{s} \sigma G q\right\rangle^{2} \int_{4 m_{c}^{2}}^{s_{0}} d s \int_{y_{i}}^{y_{f}} d y \int_{z_{i}}^{1-y} d z e^{-\frac{s}{T^{2}}}\{y z\}\left\{\frac{\left(s-\bar{m}^{2}\right)^{2}}{12288 \pi^{6}}+\frac{s\left(s-\bar{m}^{2}\right)}{3072 \pi^{6}}\right\} \\
& -m_{c}^{2}\left\langle\bar{q} g_{s} \sigma G q\right\rangle^{2} \int_{4 m_{c}^{2}}^{s_{0}} d s \int_{y_{i}}^{y_{f}} d y \int_{z_{i}}^{1-y} d z e^{-\frac{s}{T^{2}}}\left\{-\frac{\bar{m}^{2}-s}{2048 \pi^{6}}\right\} \\
& +\left\langle\bar{q} g_{s} \sigma G q\right\rangle^{2} \int_{4 m_{c}^{2}}^{s_{0}} d s \int_{y_{i}}^{y_{f}} d y \int_{z_{i}}^{1-y} d z e^{-\frac{s}{T^{2}}}\{y z\}\left\{\frac{\left(s-\bar{m}^{2}\right)^{2}}{589824 \pi^{6}}+\frac{s\left(s-\bar{m}^{2}\right)}{147456 \pi^{6}}\right\} \\
& -m_{c}^{2}\left\langle\bar{q} g_{s} \sigma G q\right\rangle^{2} \int_{4 m_{c}^{2}}^{s_{0}} d s \int_{y_{i}}^{y_{f}} d y \int_{z_{i}}^{1-y} d z e^{-\frac{s}{T^{2}}}\left\{-\frac{\bar{m}^{2}-s}{98304 \pi^{6}}\right\} \\
& +\left\langle\bar{q} g_{s} \sigma G q\right\rangle^{2} \int_{4 m_{c}^{2}}^{s_{0}} d s \int_{y_{i}}^{y_{f}} d y \int_{z_{i}}^{1-y} d z e^{-\frac{s}{T^{2}}}\{y z\}\left\{\frac{\left(s-\bar{m}^{2}\right)^{2}}{294912 \pi^{6}}+\frac{s\left(s-\bar{m}^{2}\right)}{294912 \pi^{6}}\right\} .
\end{aligned}
$$

Type C for $J_{3}$ 


$$
\begin{aligned}
\mathbf{S}_{C}(14) & =\left\langle\bar{q} g_{s} \sigma G q\right\rangle\langle\bar{q} q\rangle^{3} \int_{y_{i}}^{y_{f}} d y e^{-\frac{\tilde{m}^{2}}{T^{2}}}\{(y-1) y\}\left\{\frac{\tilde{m}^{2}}{108 \pi^{2}}+\frac{\tilde{m}^{4}}{432 \pi^{2} T^{2}}\right\} \\
& +\left\langle\bar{q} g_{s} \sigma G q\right\rangle\langle\bar{q} q\rangle^{3} \int_{y_{i}}^{y_{f}} d y e^{-\frac{\tilde{m}^{2}}{T^{2}}}\{(y-1) y\}\left\{\frac{5 \tilde{m}^{2}}{432 \pi^{2}}+\frac{\tilde{m}^{4}}{216 \pi^{2} T^{2}}\right\} \\
& -m_{c}^{2}\left\langle\bar{q} g_{s} \sigma G q\right\rangle\langle\bar{q} q\rangle^{3} \int_{y_{i}}^{y_{f}} d y e^{-\frac{\tilde{m}^{2}}{T^{2}}}\left\{-\frac{1}{144 \pi^{2}}-\frac{\tilde{m}^{2}}{144 \pi^{2} T^{2}}\right\} \\
& +\langle\bar{q} q\rangle^{3}\left\langle\bar{q} g_{s} \sigma G q\right\rangle \int_{y_{i}}^{y_{f}} d y e^{-\frac{\tilde{m}^{2}}{T^{2}}}\{(y-1) y\}\left\{\frac{5 \tilde{m}^{2}}{23328 \pi^{4}}+\frac{\tilde{m}^{4}}{11664 \pi^{4} T^{2}}\right\} \\
& -m_{c}^{2}\langle\bar{q} q\rangle^{3}\left\langle\bar{q} g_{s} \sigma G q\right\rangle \int_{y_{i}}^{y_{f}} d y e^{-\frac{\tilde{m}^{2}}{T^{2}}}\left\{-\frac{1}{7776 \pi^{4}}-\frac{\tilde{m}^{2}}{7776 \pi^{4} T^{2}}\right\} \\
& +\langle\bar{q} q\rangle^{3}\left\langle\bar{q} g_{s} \sigma G q\right\rangle \int_{y_{i}}^{y_{f}} d y e^{-\frac{\tilde{m}^{2}}{T^{2}}}\{(y-1) y\}\left\{\frac{\tilde{m}^{2}}{5832 \pi^{4}}+\frac{\tilde{m}^{4}}{23328 \pi^{4} T^{2}}\right\},
\end{aligned}
$$

$$
\begin{aligned}
\mathbf{S}_{C}(16)_{1} & =\left\langle\bar{q} g_{s} \sigma G q\right\rangle^{2}\langle\bar{q} q\rangle^{2} \int_{y_{i}}^{y_{f}} d y e^{-\frac{\tilde{m}^{2}}{T^{2}}}\{(y-1) y\}\left\{-\frac{1}{192 \pi^{2}}-\frac{\tilde{m}^{6}}{1152 \pi^{2} T^{6}}-\frac{\tilde{m}^{4}}{384 \pi^{2} T^{4}}-\frac{\tilde{m}^{2}}{192 \pi^{2} T^{2}}\right\} \\
& +\left\langle\bar{q} g_{s} \sigma G q\right\rangle^{2}\langle\bar{q} q\rangle^{2} \int_{y_{i}}^{y_{f}} d y e^{-\frac{\tilde{m}^{2}}{T^{2}}}\{(y-1) y\}\left\{\frac{1}{192 \pi^{2}}-\frac{\tilde{m}^{6}}{576 \pi^{2} T^{6}}-\frac{\tilde{m}^{4}}{384 \pi^{2} T^{4}}\right\} \\
& -m_{c}^{2}\left\langle\bar{q} g_{s} \sigma G q\right\rangle^{2}\langle\bar{q} q\rangle^{2} \int_{y_{i}}^{y_{f}} d y e^{-\frac{\tilde{m}^{2}}{T^{2}}}\left\{\frac{\tilde{m}^{4}}{64 \pi^{2} T^{6}}\right\} \\
& +\langle\bar{q} q\rangle^{2}\left\langle\bar{q} g_{s} \sigma G q\right\rangle^{2} \int_{y_{i}}^{y_{f}} d y e^{-\frac{\tilde{m}^{2}}{T^{2}}}\{(y-1) y\}\left\{\frac{1}{31104 \pi^{4}}-\frac{\tilde{m}^{6}}{93312 \pi^{4} T^{6}}-\frac{\tilde{m}^{4}}{62208 \pi^{4} T^{4}}\right\} \\
& -m_{c}^{2}\langle\bar{q} q\rangle^{2}\left\langle\bar{q} g_{s} \sigma G q\right\rangle^{2} \int_{y_{i}}^{y_{f}} d y e^{-\frac{\tilde{m}^{2}}{T^{2}}}\left\{\frac{\tilde{m}^{4}}{62208 \pi^{4} T^{6}}\right\} \\
& +\langle\bar{q} q\rangle^{2}\left\langle\bar{q} g_{s} \sigma G q\right\rangle^{2} \int_{y_{i}}^{y_{f}} d y e^{-\frac{\tilde{m}^{2}}{T^{2}}}\{(y-1) y\}\left\{-\frac{1}{31104 \pi^{4}}-\frac{\tilde{m}^{6}}{186624 \pi^{4} T^{6}}-\frac{\tilde{m}^{4}}{62208 \pi^{4} T^{4}}-\frac{\tilde{m}^{2}}{31104 \pi^{4} T^{2}}\right\}
\end{aligned}
$$




$$
\begin{aligned}
\mathbf{S}_{C}(16)_{2} & =m_{c}^{2}\langle\bar{q} q\rangle^{4}\left\langle g_{s}^{2} G G\right\rangle \int_{y_{i}}^{y_{f}} d y e^{-\frac{\tilde{m}^{2}}{T^{2}}}\left\{\frac{y-1}{y^{2}}\right\}\left\{\frac{\tilde{m}^{2}}{7776 \pi^{2} T^{4}}-\frac{1}{5184 \pi^{2} T^{2}}\right\} \\
& -m_{c}^{2}\langle\bar{q} q\rangle^{4}\left\langle g_{s}^{2} G G\right\rangle \int_{y_{i}}^{y_{f}} d y e^{-\frac{\tilde{m}^{2}}{T^{2}}}\left\{\frac{1}{y^{2}}\right\}\left\{\frac{1}{1728 \pi^{2} T^{2}}\right\} \\
& -m_{c}^{4}\langle\bar{q} q\rangle^{4}\left\langle g_{s}^{2} G G\right\rangle \int_{y_{i}}^{y_{f}} d y e^{-\frac{\tilde{m}^{2}}{T^{2}}}\left\{\frac{1}{y^{3}}\right\}\left\{-\frac{1}{5184 \pi^{2} T^{4}}\right\} \\
& +m_{c}^{2}\left\langle g_{s}^{2} G G\right\rangle\langle\bar{q} q\rangle^{4} \int_{y_{i}}^{y_{f}} d y e^{-\frac{\tilde{m}^{2}}{T^{2}}}\left\{\frac{y-1}{y^{2}}\right\}\left\{\frac{\tilde{m}^{2}}{15552 \pi^{2} T^{4}}\right\} \\
& +\langle\bar{q} q\rangle^{4}\left\langle g_{s}^{2} G G\right\rangle \int_{y_{i}}^{y_{f}} d y e^{-\frac{\tilde{m}^{2}}{T^{2}}}\{(y-1) y\}\left\{\frac{1}{2592 \pi^{2}}-\frac{\tilde{m}^{6}}{7776 \pi^{2} T^{6}}-\frac{\tilde{m}^{4}}{5184 \pi^{2} T^{4}}\right\} \\
& -m_{c}^{2}\langle\bar{q} q\rangle^{4}\left\langle g_{s}^{2} G G\right\rangle \int_{y_{i}}^{y_{f}} d y e^{-\frac{\tilde{m}^{2}}{T^{2}}}\left\{\frac{\tilde{m}^{4}}{5184 \pi^{2} T^{6}}\right\} \\
& +\langle\bar{q} q\rangle^{4}\left\langle g_{s}^{2} G G\right\rangle \int_{y_{i}}^{y_{f}} d y e^{-\frac{\tilde{m}^{2}}{T^{2}}}\{(y-1) y\}\left\{-\frac{1}{2592 \pi^{2}}-\frac{\tilde{m}^{6}}{15552 \pi^{2} T^{6}}-\frac{\tilde{m}^{4}}{5184 \pi^{2} T^{4}}-\frac{\tilde{m}^{2}}{2592 \pi^{2} T^{2}}\right\} .
\end{aligned}
$$


Type A for $J_{4}$

$$
\begin{aligned}
\mathbf{S}_{A}(12) & =\langle\bar{q} q\rangle^{4} \int_{4 m_{c}^{2}}^{s_{0}} d s \int_{y_{i}}^{y_{f}} d y e^{-\frac{s}{T^{2}}}\{(y-1) y\}\left\{\frac{\tilde{m}^{2}-s}{216 \pi^{2}}-\frac{s}{432 \pi^{2}}\right\} \\
& +\langle\bar{q} q\rangle^{4} \int_{4 m_{c}^{2}}^{s_{0}} d s \int_{y_{i}}^{y_{f}} d y e^{-\frac{s}{T^{2}}}\{(y-1) y\}\left\{\frac{\tilde{m}^{2}-s}{432 \pi^{2}}-\frac{s}{216 \pi^{2}}\right\} \\
& +m_{c}^{2}\langle\bar{q} q\rangle^{4} \int_{4 m_{c}^{2}}^{s_{0}} d s \int_{y_{i}}^{y_{f}} d y e^{-\frac{s}{T^{2}}}\left\{\frac{1}{144 \pi^{2}}\right\} \\
& +\langle\bar{q} q\rangle^{4} \int_{4 m_{c}^{2}}^{s_{0}} d s \int_{y_{i}}^{y_{f}} d y e^{-\frac{s}{T^{2}}}\{(y-1) y\}\left\{\frac{\tilde{m}^{2}-s}{11664 \pi^{4}}-\frac{s}{5832 \pi^{4}}\right\} \\
& +m_{c}^{2}\langle\bar{q} q\rangle^{4} \int_{4 m_{c}^{2}}^{s_{0}} d s \int_{y_{i}}^{y_{f}} d y e^{-\frac{s}{T^{2}}}\left\{\frac{1}{3888 \pi^{4}}\right\} \\
& +\langle\bar{q} q\rangle^{4} \int_{4 m_{c}^{2}}^{s_{0}} d s \int_{y_{i}}^{y_{f}} d y e^{-\frac{s}{T^{2}}}\{(y-1) y\}\left\{\frac{\tilde{m}^{2}-s}{5832 \pi^{4}}-\frac{s}{11664 \pi^{4}}\right\},
\end{aligned}
$$

$$
\begin{aligned}
\mathbf{S}_{A}(14) & =\left\langle\bar{q} g_{s} \sigma G q\right\rangle\langle\bar{q} q\rangle^{3} \int_{4 m_{c}^{2}}^{s_{0}} d s \int_{y_{i}}^{y_{f}} d y e^{-\frac{s}{T^{2}}}\{(y-1) y\}\left\{\frac{1}{72 \pi^{2}}\right\} \\
& +\left\langle\bar{q} g_{s} \sigma G q\right\rangle\langle\bar{q} q\rangle^{3} \int_{4 m_{c}^{2}}^{s_{0}} d s \int_{y_{i}}^{y_{f}} d y e^{-\frac{s}{T^{2}}}\{(y-1) y\}\left\{\frac{1}{144 \pi^{2}}\right\} \\
& +\langle\bar{q} q\rangle^{3}\left\langle\bar{q} g_{s} \sigma G q\right\rangle \int_{4 m_{c}^{2}}^{s_{0}} d s \int_{y_{i}}^{y_{f}} d y e^{-\frac{s}{T^{2}}}\{(y-1) y\}\left\{\frac{1}{7776 \pi^{4}}\right\} \\
& +\langle\bar{q} q\rangle^{3}\left\langle\bar{q} g_{s} \sigma G q\right\rangle \int_{4 m_{c}^{2}}^{s_{0}} d s \int_{y_{i}}^{y_{f}} d y e^{-\frac{s}{T^{2}}}\{(y-1) y\}\left\{\frac{1}{3888 \pi^{4}}\right\} .
\end{aligned}
$$

Type B for $J_{4}$

$$
\begin{aligned}
\mathbf{S}_{B}(0) & =\int_{4 m_{c}^{2}}^{s_{0}} d s \int_{y_{i}}^{y_{f}} d y \int_{z_{i}}^{1-y} d z e^{-\frac{s}{T^{2}}}\left\{-y z(y+z-1)^{5}\right\}\left\{\frac{\left(s-\bar{m}^{2}\right)^{7}}{412876800 \pi^{10}}+\frac{s\left(s-\bar{m}^{2}\right)^{6}}{117964800 \pi^{10}}\right\} \\
& +\int_{4 m_{c}^{2}}^{s_{0}} d s \int_{y_{i}}^{y_{f}} d y \int_{z_{i}}^{1-y} d z e^{-\frac{s}{T^{2}}}\left\{-y z(y+z-1)^{5}\right\}\left\{\frac{\left(s-\bar{m}^{2}\right)^{7}}{825753600 \pi^{10}}+\frac{s\left(s-\bar{m}^{2}\right)^{6}}{58982400 \pi^{10}}\right\} \\
& +m_{c}^{2} \int_{4 m_{c}^{2}}^{s_{0}} d s \int_{y_{i}}^{y_{f}} d y \int_{z_{i}}^{1-y} d z e^{-\frac{s}{T^{2}}}\left\{-(y+z-1)^{5}\right\}\left\{\frac{\left(s-\bar{m}^{2}\right)^{6}}{39321600 \pi^{10}}\right\}
\end{aligned}
$$




$$
\begin{aligned}
\mathbf{S}_{B}(4) & =m_{c}^{2}\left\langle g_{s}^{2} G G\right\rangle \int_{4 m_{c}^{2}}^{s_{0}} d s \int_{y_{i}}^{y_{f}} d y \int_{z_{i}}^{1-y} d z e^{-\frac{s}{T^{2}}}\left\{-\frac{z(y+z-1)^{5}}{y^{2}}\right\}\left\{-\frac{\left(s-\bar{m}^{2}\right)^{4}}{141557760 \pi^{10}}-\frac{s\left(s-\bar{m}^{2}\right)^{3}}{17694720 \pi^{10}}\right\} \\
& +m_{c}^{2}\left\langle g_{s}^{2} G G\right\rangle \int_{4 m_{c}^{2}}^{s_{0}} d s \int_{y_{i}}^{y_{f}} d y \int_{z_{i}}^{1-y} d z e^{-\frac{s}{T^{2}}}\left\{\frac{(y+z-1)^{5}}{y^{2}}\right\}\left\{\frac{s y\left(s-\bar{m}^{2}\right)^{3}}{11796480 \pi^{10}}-\frac{(1-y)\left(s-\bar{m}^{2}\right)^{4}}{23592960 \pi^{10}}\right\} \\
& +m_{c}^{2}\left\langle g_{s}^{2} G G\right\rangle \int_{4 m_{c}^{2}}^{s_{0}} d s \int_{y_{i}}^{y_{f}} d y \int_{z_{i}}^{1-y} d z e^{-\frac{s}{T^{2}}}\left\{-\frac{z(y+z-1)^{5}}{y^{2}}\right\}\left\{-\frac{\left(s-\bar{m}^{2}\right)^{4}}{70778880 \pi^{10}}-\frac{s\left(s-\bar{m}^{2}\right)^{3}}{35389440 \pi^{10}}\right\} \\
& +\left\langle g_{s}^{2} G G\right\rangle \int_{4 m_{c}^{2}}^{s_{0}} d s \int_{y_{i}}^{y_{f}} d y \int_{z_{i}}^{1-y} d z e^{-\frac{s}{T^{2}}}\left\{-y z(y+z-1)^{3}\right\}\left\{\frac{\left(s-\bar{m}^{2}\right)^{5}}{23592960 \pi^{10}}+\frac{s\left(s-\bar{m}^{2}\right)^{4}}{2359296 \pi^{10}}\right\} \\
& +m_{c}^{2}\left\langle g_{s}^{2} G G\right\rangle \int_{4 m_{c}^{2}}^{s_{0}} d s \int_{y_{i}}^{y_{f}} d y \int_{z_{i}}^{1-y} d z e^{-\frac{s}{T^{2}}}\left\{-(y+z-1)^{3}\right\}\left\{\frac{\left(s-\bar{m}^{2}\right)^{4}}{1572864 \pi^{10}}\right\} \\
& +\left\langle g_{s}^{2} G G\right\rangle \int_{4 m_{c}^{2}}^{s_{0}} d s \int_{y_{i}}^{y_{f}} d y \int_{z_{i}}^{1-y} d z e^{-\frac{s}{T^{2}}}\left\{-y z(y+z-1)^{3}\right\}\left\{\frac{\left(s-\bar{m}^{2}\right)^{5}}{11796480 \pi^{10}}+\frac{s\left(s-\bar{m}^{2}\right)^{4}}{4718592 \pi^{10}}\right\}
\end{aligned}
$$

$$
\begin{aligned}
\mathbf{S}_{B}(6)_{1} & =\langle\bar{q} q\rangle^{2} \int_{4 m_{c}^{2}}^{s_{0}} d s \int_{y_{i}}^{y_{f}} d y \int_{z_{i}}^{1-y} d z e^{-\frac{s}{T^{2}}}\left\{y z(y+z-1)^{2}\right\}\left\{\frac{\left(s-\bar{m}^{2}\right)^{4}}{9216 \pi^{6}}+\frac{s\left(s-\bar{m}^{2}\right)^{3}}{4608 \pi^{6}}\right\} \\
& +\langle\bar{q} q\rangle^{2} \int_{4 m_{c}^{2}}^{s_{0}} d s \int_{y_{i}}^{y_{f}} d y \int_{z_{i}}^{1-y} d z e^{-\frac{s}{T^{2}}}\left\{y z(y+z-1)^{2}\right\}\left\{\frac{\left(s-\bar{m}^{2}\right)^{4}}{18432 \pi^{6}}+\frac{s\left(s-\bar{m}^{2}\right)^{3}}{2304 \pi^{6}}\right\} \\
& +m_{c}^{2}\langle\bar{q} q\rangle^{2} \int_{4 m_{c}^{2}}^{s_{0}} d s \int_{y_{i}}^{y_{f}} d y \int_{z_{i}}^{1-y} d z e^{-\frac{s}{T^{2}}}\left\{(y+z-1)^{2}\right\}\left\{\frac{\left(s-\bar{m}^{2}\right)^{3}}{1536 \pi^{6}}\right\} \\
& +\langle\bar{q} q\rangle^{2} \int_{4 m_{c}^{2}}^{s_{0}} d s \int_{y_{i}}^{y_{f}} d y \int_{z_{i}}^{1-y} d z e^{-\frac{s}{T^{2}}}\left\{y z(y+z-1)^{2}\right\}\left\{\frac{\left(s-\bar{m}^{2}\right)^{4}}{995328 \pi^{8}}+\frac{s\left(s-\bar{m}^{2}\right)^{3}}{124416 \pi^{8}}\right\} \\
& +m_{c}^{2}\langle\bar{q} q\rangle^{2} \int_{4 m_{c}^{2}}^{s_{0}} d s \int_{y_{i}}^{y_{f}} d y \int_{z_{i}}^{1-y} d z e^{-\frac{s}{T^{2}}}\left\{(y+z-1)^{2}\right\}\left\{\frac{\left(s-\bar{m}^{2}\right)^{3}}{82944 \pi^{8}}\right\} \\
& +\langle\bar{q} q\rangle^{2} \int_{4 m_{c}^{2}}^{s_{0}} d s \int_{y_{i}}^{y_{f}} d y \int_{z_{i}}^{1-y} d z e^{-\frac{s}{T^{2}}}\left\{y z(y+z-1)^{2}\right\}\left\{\frac{\left(s-\bar{m}^{2}\right)^{4}}{497664 \pi^{8}}+\frac{s\left(s-\bar{m}^{2}\right)^{3}}{248832 \pi^{8}}\right\},
\end{aligned}
$$




$$
\begin{aligned}
& \mathbf{S}_{B}(6)_{2}=m_{c}^{2}\langle\bar{q} q\rangle^{2} \int_{4 m_{c}^{2}}^{s_{0}} d s \int_{y_{i}}^{y_{f}} d y \int_{z_{i}}^{1-y} d z e^{-\frac{s}{T^{2}}}\left\{\frac{(y+z-1)^{4}}{y}\right\}\left\{-\frac{\left(s-\bar{m}^{2}\right)^{3}}{331776 \pi^{8}}-\frac{s\left(s-\bar{m}^{2}\right)^{2}}{221184 \pi^{8}}\right\} \\
& +m_{c}^{2}\langle\bar{q} q\rangle^{2} \int_{4 m_{c}^{2}}^{s_{0}} d s \int_{y_{i}}^{y_{f}} d y \int_{z_{i}}^{1-y} d z e^{-\frac{s}{T^{2}}}\left\{\frac{z(y+z-1)^{4}}{y^{2}}\right\}\left\{-\frac{\left(s-\bar{m}^{2}\right)^{3}}{995328 \pi^{8}}-\frac{s\left(s-\bar{m}^{2}\right)^{2}}{663552 \pi^{8}}\right\} \\
& +m_{c}^{2}\langle\bar{q} q\rangle^{2} \int_{4 m_{c}^{2}}^{s_{0}} d s \int_{y_{i}}^{y_{f}} d y \int_{z_{i}}^{1-y} d z e^{-\frac{s}{T^{2}}}\left\{\frac{z(y+z-1)^{4}}{y^{2}}\right\}\left\{-\frac{\left(s-\bar{m}^{2}\right)^{3}}{1990656 \pi^{8}}-\frac{s\left(s-\bar{m}^{2}\right)^{2}}{331776 \pi^{8}}\right\} \\
& +\langle\bar{q} q\rangle^{2} \int_{4 m_{c}^{2}}^{s_{0}} d s \int_{y_{i}}^{y_{f}} d y \int_{z_{i}}^{1-y} d z e^{-\frac{s}{T^{2}}}\left\{\frac{z(y+z-1)^{4}}{y}\right\}\left\{\frac{s^{2} y\left(s-\bar{m}^{2}\right)^{2}}{995328 \pi^{8}}+\frac{y\left(s-\bar{m}^{2}\right)^{4}}{1990656 \pi^{8}}\right\} \\
& +\langle\bar{q} q\rangle^{2} \int_{4 m_{c}^{2}}^{s_{0}} d s \int_{y_{i}}^{y_{f}} d y \int_{z_{i}}^{1-y} d z e^{-\frac{s}{T^{2}}}\left\{\frac{z(y+z-1)^{4}}{y}\right\}\left\{\frac{s y\left(s-\bar{m}^{2}\right)^{3}}{497664 \pi^{8}}-\frac{\left(s-\bar{m}^{2}\right)^{4}}{2985984 \pi^{8}}-\frac{s\left(s-\bar{m}^{2}\right)^{3}}{1492992 \pi^{8}}\right\} \\
& +\langle\bar{q} q\rangle^{2} \int_{4 m_{c}^{2}}^{s_{0}} d s \int_{y_{i}}^{y_{f}} d y \int_{z_{i}}^{1-y} d z e^{-\frac{s}{T^{2}}}\left\{\frac{z(y+z-1)^{4}}{y}\right\}\left\{\frac{s^{2} y\left(s-\bar{m}^{2}\right)^{2}}{497664 \pi^{8}}+\frac{y\left(s-\bar{m}^{2}\right)^{4}}{3981312 \pi^{8}}\right\} \\
& +\langle\bar{q} q\rangle^{2} \int_{4 m_{c}^{2}}^{s_{0}} d s \int_{y_{i}}^{y_{f}} d y \int_{z_{i}}^{1-y} d z e^{-\frac{s}{T^{2}}}\left\{\frac{z(y+z-1)^{4}}{y}\right\}\left\{\frac{s y\left(s-\bar{m}^{2}\right)^{3}}{331776 \pi^{8}}-\frac{\left(s-\bar{m}^{2}\right)^{4}}{5971968 \pi^{8}}-\frac{s\left(s-\bar{m}^{2}\right)^{3}}{746496 \pi^{8}}\right\} \\
& +\langle\bar{q} q\rangle^{2} \int_{4 m_{c}^{2}}^{s_{0}} d s \int_{y_{i}}^{y_{f}} d y \int_{z_{i}}^{1-y} d z e^{-\frac{s}{T^{2}}}\left\{\frac{z(y+z-1)^{4}}{y}\right\}\left\{\frac{s^{2} y\left(s-\bar{m}^{2}\right)^{2}}{995328 \pi^{8}}-\frac{y\left(s-\bar{m}^{2}\right)^{4}}{1990656 \pi^{8}}\right\} \\
& +\langle\bar{q} q\rangle^{2} \int_{4 m_{c}^{2}}^{s_{0}} d s \int_{y_{i}}^{y_{f}} d y \int_{z_{i}}^{1-y} d z e^{-\frac{s}{T^{2}}}\left\{\frac{z(y+z-1)^{4}}{y}\right\}\left\{-\frac{s y\left(s-\bar{m}^{2}\right)^{3}}{497664 \pi^{8}}+\frac{\left(s-\bar{m}^{2}\right)^{4}}{11943936 \pi^{8}}+\frac{s\left(s-\bar{m}^{2}\right)^{3}}{5971968 \pi^{8}}\right\} \\
& +\langle\bar{q} q\rangle^{2} \int_{4 m_{c}^{2}}^{s_{0}} d s \int_{y_{i}}^{y_{f}} d y \int_{z_{i}}^{1-y} d z e^{-\frac{s}{T^{2}}}\left\{\frac{z(y+z-1)^{4}}{y}\right\}\left\{\frac{s^{2} y\left(s-\bar{m}^{2}\right)^{2}}{497664 \pi^{8}}-\frac{y\left(s-\bar{m}^{2}\right)^{4}}{3981312 \pi^{8}}\right\} \\
& +\langle\bar{q} q\rangle^{2} \int_{4 m_{c}^{2}}^{s_{0}} d s \int_{y_{i}}^{y_{f}} d y \int_{z_{i}}^{1-y} d z e^{-\frac{s}{T^{2}}}\left\{\frac{z(y+z-1)^{4}}{y}\right\}\left\{-\frac{s y\left(s-\bar{m}^{2}\right)^{3}}{331776 \pi^{8}}+\frac{\left(s-\bar{m}^{2}\right)^{4}}{23887872 \pi^{8}}+\frac{s\left(s-\bar{m}^{2}\right)^{3}}{2985984 \pi^{8}}\right\},
\end{aligned}
$$

$$
\begin{aligned}
\mathbf{S}_{B}(8) & =\left\langle\bar{q} g_{s} \sigma G q\right\rangle\langle\bar{q} q\rangle \int_{4 m_{c}^{2}}^{s_{0}} d s \int_{y_{i}}^{y_{f}} d y \int_{z_{i}}^{1-y} d z e^{-\frac{s}{T^{2}}}\{-y z(y+z-1)\}\left\{-\frac{\left(s-\bar{m}^{2}\right)^{3}}{2304 \pi^{6}}-\frac{s\left(s-\bar{m}^{2}\right)^{2}}{1536 \pi^{6}}\right\} \\
& +\left\langle\bar{q} g_{s} \sigma G q\right\rangle\langle\bar{q} q\rangle \int_{4 m_{c}^{2}}^{s_{0}} d s \int_{y_{i}}^{y_{f}} d y \int_{z_{i}}^{1-y} d z e^{-\frac{s}{T^{2}}}\{-y z(y+z-1)\}\left\{-\frac{\left(s-\bar{m}^{2}\right)^{3}}{4608 \pi^{6}}-\frac{s\left(s-\bar{m}^{2}\right)^{2}}{768 \pi^{6}}\right\} \\
& +m_{c}^{2}\left\langle\bar{q} g_{s} \sigma G q\right\rangle\langle\bar{q} q\rangle \int_{4 m_{c}^{2}}^{s_{0}} d s \int_{y_{i}}^{y_{f}} d y \int_{z_{i}}^{1-y} d z e^{-\frac{s}{T^{2}}}\{-y-z+1\}\left\{-\frac{\left(s-\bar{m}^{2}\right)^{2}}{512 \pi^{6}}\right\}
\end{aligned}
$$




$$
\begin{aligned}
\mathbf{S}_{B}(10)_{1} & =m_{c}^{2}\langle\bar{q} q\rangle^{2}\left\langle g_{s}^{2} G G\right\rangle \int_{4 m_{c}^{2}}^{s_{0}} d s \int_{y_{i}}^{y_{f}} d y \int_{z_{i}}^{1-y} d z e^{-\frac{s}{T^{2}}}\left\{\frac{z(y+z-1)^{2}}{y^{2}}\right\}\left\{\frac{\bar{m}^{2}-s}{27648 \pi^{6}}-\frac{s}{13824 \pi^{6}}\right\} \\
& +m_{c}^{2}\left\langle g_{s}^{2} G G\right\rangle\langle\bar{q} q\rangle^{2} \int_{4 m_{c}^{2}}^{s_{0}} d s \int_{y_{i}}^{y_{f}} d y \int_{z_{i}}^{1-y} d z e^{-\frac{s}{T^{2}}}\left\{-\frac{(y+z-1)^{2}}{y^{2}}\right\}\left\{\frac{(1-y)\left(\bar{m}^{2}-s\right)}{4608 \pi^{6}}+\frac{s y}{9216 \pi^{6}}\right\} \\
& +m_{c}^{2}\left\langle g_{s}^{2} G G\right\rangle\langle\bar{q} q\rangle^{2} \int_{4 m_{c}^{2}}^{s_{0}} d s \int_{y_{i}}^{y_{f}} d y \int_{z_{i}}^{1-y} d z e^{-\frac{s}{T^{2}}}\left\{\frac{z(y+z-1)^{2}}{y^{2}}\right\}\left\{\frac{\bar{m}^{2}-s}{13824 \pi^{6}}-\frac{s}{27648 \pi^{6}}\right\} \\
& +\langle\bar{q} q\rangle^{2}\left\langle g_{s}^{2} G G\right\rangle \int_{4 m_{c}^{2}}^{s_{0}} d s \int_{y_{i}}^{y_{f}} d y \int_{z_{i}}^{1-y} d z e^{-\frac{s}{T^{2}}}\{y z\}\left\{\frac{\left(s-\bar{m}^{2}\right)^{2}}{36864 \pi^{6}}+\frac{s\left(s-\bar{m}^{2}\right)}{9216 \pi^{6}}\right\} \\
& +m_{c}^{2}\left\langle g_{s}^{2} G G\right\rangle\langle\bar{q} q\rangle^{2} \int_{4 m_{c}^{2}}^{s_{0}} d s \int_{y_{i}}^{y_{f}} d y \int_{z_{i}}^{1-y} d z e^{-\frac{s}{T^{2}}}\left\{-\frac{\bar{m}^{2}-s}{6144 \pi^{6}}\right\} \\
& +\left\langle g_{s}^{2} G G\right\rangle\langle\bar{q} q\rangle^{2} \int_{4 m_{c}^{2}}^{s_{0}} d s \int_{y_{i}}^{y_{f}} d y \int_{z_{i}}^{1-y} d z e^{-\frac{s}{T^{2}}}\{y z\}\left\{\frac{\left(s-\bar{m}^{2}\right)^{2}}{18432 \pi^{6}}+\frac{s\left(s-\bar{m}^{2}\right)}{18432 \pi^{6}}\right\} \\
& +\langle\bar{q} q\rangle^{2}\left\langle g_{s}^{2} G G\right\rangle \int_{4 m_{c}^{2}}^{s_{0}} d s \int_{y_{i}}^{y_{f}} d y \int_{z_{i}}^{1-y} d z e^{-\frac{s}{T^{2}}}\{y z\}\left\{\frac{\left(s-\bar{m}^{2}\right)^{2}}{55296 \pi^{6}}+\frac{s\left(s-\bar{m}^{2}\right)}{13824 \pi^{6}}\right\} \\
& +m_{c}^{2}\langle\bar{q} q\rangle^{2}\left\langle g_{s}^{2} G G\right\rangle \int_{4 m_{c}^{2}}^{s_{0}} d s \int_{y_{i}}^{y_{f}} d y \int_{z_{i}}^{1-y} d z e^{-\frac{s}{T^{2}}}\left\{-\frac{\bar{m}^{2}-s}{9216 \pi^{6}}\right\} \\
& +\langle\bar{q} q\rangle^{2}\left\langle g_{s}^{2} G G\right\rangle \int_{4 m_{c}^{2}}^{s_{0}} d s \int_{y_{i}}^{y_{f}} d y \int_{z_{i}}^{1-y} d z e^{-\frac{s}{T^{2}}}\{y z\}\left\{\frac{\left(s-\bar{m}^{2}\right)^{2}}{27648 \pi^{6}}+\frac{s\left(s-\bar{m}^{2}\right)}{27648 \pi^{6}}\right\}
\end{aligned}
$$

$$
\begin{aligned}
\mathbf{S}_{B}(10)_{2} \quad & +\left\langle\bar{q} g_{s} \sigma G q\right\rangle^{2} \int_{4 m_{c}^{2}}^{s_{0}} d s \int_{y_{i}}^{y_{f}} d y \int_{z_{i}}^{1-y} d z e^{-\frac{s}{T^{2}}}\left\{(y z\}\left\{\frac{\left(s-\bar{m}^{2}\right)^{2}}{6144 \pi^{6}}+\frac{s\left(s-\bar{m}^{2}\right)}{6144 \pi^{6}}\right\}\right. \\
& +\left\langle\bar{q} g_{s} \sigma G q\right\rangle^{2} \int_{4 m_{c}^{2}}^{s_{0}} d s \int_{y_{i}}^{y_{f}} d y \int_{z_{i}}^{1-y} d z e^{-\frac{s}{T^{2}}}\{y z\}\left\{\frac{\left(s-\bar{m}^{2}\right)^{2}}{12288 \pi^{6}}+\frac{s\left(s-\bar{m}^{2}\right)}{3072 \pi^{6}}\right\} \\
& +m_{c}^{2}\left\langle\bar{q} g_{s} \sigma G q\right\rangle^{2} \int_{4 m_{c}^{2}}^{s_{0}} d s \int_{y_{i}}^{y_{f}} d y \int_{z_{i}}^{1-y} d z e^{-\frac{s}{T^{2}}}\left\{-\frac{\bar{m}^{2}-s}{2048 \pi^{6}}\right\} \\
& +\left\langle\bar{q} g_{s} \sigma G q\right\rangle^{2} \int_{4 m_{c}^{2}}^{s_{0}} d s \int_{y_{i}}^{y_{f}} d y \int_{z_{i}}^{1-y} d z e^{-\frac{s}{T^{2}}}\{y z\}\left\{\frac{\left(s-\bar{m}^{2}\right)^{2}}{589824 \pi^{6}}+\frac{s\left(s-\bar{m}^{2}\right)}{147456 \pi^{6}}\right\} \\
& +m_{c}^{2}\left\langle\bar{q} g_{s} \sigma G q\right\rangle^{2} \int_{4 m_{c}^{2}}^{s_{0}} d s \int_{y_{i}}^{y_{f}} d y \int_{z_{i}}^{1-y} d z e^{-\frac{s}{T^{2}}}\left\{-\frac{\bar{m}^{2}-s}{98304 \pi^{6}}\right\} \\
& +\left\langle\bar{q} g_{s} \sigma G q\right\rangle^{2} \int_{4 m_{c}^{2}}^{s_{0}} d s \int_{y_{i}}^{y_{f}} d y \int_{z_{i}}^{1-y} d z e^{-\frac{s}{T^{2}}}\{y z\}\left\{\frac{\left(s-\bar{m}^{2}\right)^{2}}{294912 \pi^{6}}+\frac{s\left(s-\bar{m}^{2}\right)}{294912 \pi^{6}}\right\}
\end{aligned}
$$




$$
\begin{aligned}
& \mathbf{S}_{B}(12)=m_{c}^{2}\langle\bar{q} q\rangle^{4} \int_{4 m_{c}^{2}}^{s_{0}} d s \int_{y_{i}}^{y_{f}} d y \int_{z_{i}}^{1-y} d z e^{-\frac{s}{T^{2}}}\left\{\frac{y+z-1}{y}\right\}\left\{\frac{1}{1296 \pi^{4}}\right\} \\
& +m_{c}^{2}\langle\bar{q} q\rangle^{4} \int_{4 m_{c}^{2}}^{s_{0}} d s \int_{y_{i}}^{y_{f}} d y \int_{z_{i}}^{1-y} d z e^{-\frac{s}{T^{2}}}\left\{-\frac{z(y+z-1)}{y^{2}}\right\}\left\{-\frac{1}{3888 \pi^{4}}\right\} \\
& +m_{c}^{2}\langle\bar{q} q\rangle^{4} \int_{4 m_{c}^{2}}^{s_{0}} d s \int_{y_{i}}^{y_{f}} d y \int_{z_{i}}^{1-y} d z e^{-\frac{s}{T^{2}}}\left\{-\frac{z(y+z-1)}{y^{2}}\right\}\left\{-\frac{1}{7776 \pi^{4}}\right\} \\
& +\langle\bar{q} q\rangle^{4} \int_{4 m_{c}^{2}}^{s_{0}} d s \int_{y_{i}}^{y_{f}} d y \int_{z_{i}}^{1-y} d z e^{-\frac{s}{T^{2}}}\left\{-\frac{z(y+z-1)}{y}\right\}\left\{\frac{\bar{m}^{2}-s}{2916 \pi^{4}}-\frac{s}{5832 \pi^{4}}\right\} \\
& \left.+m_{c}^{2}\langle\bar{q} q\rangle^{4} \int_{4 m_{c}^{2}}^{s_{0}} d s \int_{y_{i}}^{y_{f}} d y \int_{z_{i}}^{1-y} d z e^{-\frac{s}{T^{2}}}\left\{-\frac{z(y+z-1)}{y^{2}}\right)\right\}\left\{\frac{1}{5832 \pi^{4}}\right\} \\
& \left.+\langle\bar{q} q\rangle^{4} \int_{4 m_{c}^{2}}^{s_{0}} d s \int_{y_{i}}^{y_{f}} d y \int_{z_{i}}^{1-y} d z e^{-\frac{s}{T^{2}}}\left\{-\frac{z(y+z-1)}{y}\right)\right\}\left\{\frac{\bar{m}^{2}-s}{5832 \pi^{4}}-\frac{s}{2916 \pi^{4}}\right\} \\
& +m_{c}^{2}\langle\bar{q} q\rangle^{4} \int_{4 m_{c}^{2}}^{s_{0}} d s \int_{y_{i}}^{y_{f}} d y \int_{z_{i}}^{1-y} d z e^{-\frac{s}{T^{2}}}\left\{-\frac{z(y+z-1)}{y^{2}}\right\}\left\{\frac{1}{11664 \pi^{4}}\right\} \\
& \left.+\langle\bar{q} q\rangle^{4} \int_{4 m_{c}^{2}}^{s_{0}} d s \int_{y_{i}}^{y_{f}} d y \int_{z_{i}}^{1-y} d z e^{-\frac{s}{T^{2}}}\left\{-\frac{z(y+z-1)}{y}\right)\right\}\left\{\frac{\bar{m}^{2} y}{1944 \pi^{4}}-\frac{\bar{m}^{2}}{11664 \pi^{4}}-\frac{s y}{972 \pi^{4}}+\frac{s}{7776 \pi^{4}}\right\} \\
& +\langle\bar{q} q\rangle^{4} \int_{4 m_{c}^{2}}^{s_{0}} d s \int_{y_{i}}^{y_{f}} d y \int_{z_{i}}^{1-y} d z e^{-\frac{s}{T^{2}}}\left\{-\frac{z(y+z-1)}{y}\right\}\left\{\frac{\bar{m}^{2} y}{3888 \pi^{4}}-\frac{\bar{m}^{2}}{23328 \pi^{4}}-\frac{s y}{972 \pi^{4}}+\frac{s}{7776 \pi^{4}}\right\} .
\end{aligned}
$$

Type C for $J_{4}$

$$
\begin{aligned}
\mathbf{S}_{C}(14) & =\left\langle\bar{q} g_{s} \sigma G q\right\rangle\langle\bar{q} q\rangle^{3} \int_{y_{i}}^{y_{f}} d y e^{-\frac{\tilde{m}^{2}}{T^{2}}}\{(y-1) y\}\left\{\frac{\tilde{m}^{2}}{108 \pi^{2}}+\frac{\tilde{m}^{4}}{432 \pi^{2} T^{2}}\right\} \\
& +\left\langle\bar{q} g_{s} \sigma G q\right\rangle\langle\bar{q} q\rangle^{3} \int_{y_{i}}^{y_{f}} d y e^{-\frac{\tilde{m}^{2}}{T^{2}}}\{(y-1) y\}\left\{\frac{5 \tilde{m}^{2}}{432 \pi^{2}}+\frac{\tilde{m}^{4}}{216 \pi^{2} T^{2}}\right\} \\
& +m_{c}^{2}\left\langle\bar{q} g_{s} \sigma G q\right\rangle\langle\bar{q} q\rangle^{3} \int_{y_{i}}^{y_{f}} d y e^{-\frac{\tilde{m}^{2}}{T^{2}}}\left\{-\frac{1}{144 \pi^{2}}-\frac{\tilde{m}^{2}}{144 \pi^{2} T^{2}}\right\} \\
& +\langle\bar{q} q\rangle^{3}\left\langle\bar{q} g_{s} \sigma G q\right\rangle \int_{y_{i}}^{y_{f}} d y e^{-\frac{\tilde{m}^{2}}{T^{2}}}\{(y-1) y\}\left\{\frac{5 \tilde{m}^{2}}{23328 \pi^{4}}+\frac{\tilde{m}^{4}}{11664 \pi^{4} T^{2}}\right\} \\
& +m_{c}^{2}\langle\bar{q} q\rangle^{3}\left\langle\bar{q} g_{s} \sigma G q\right\rangle \int_{y_{i}}^{y_{f}} d y e^{-\frac{\tilde{m}^{2}}{T^{2}}}\left\{-\frac{1}{7776 \pi^{4}}-\frac{\tilde{m}^{2}}{7776 \pi^{4} T^{2}}\right\} \\
& +\langle\bar{q} q\rangle^{3}\left\langle\bar{q} g_{s} \sigma G q\right\rangle \int_{y_{i}}^{y_{f}} d y e^{-\frac{\tilde{m}^{2}}{T^{2}}}\{(y-1) y\}\left\{\frac{\tilde{m}^{2}}{5832 \pi^{4}}+\frac{\tilde{m}^{4}}{23328 \pi^{4} T^{2}}\right\},
\end{aligned}
$$




$$
\begin{aligned}
\mathbf{S}_{C}(16)_{1} & =\left\langle\bar{q} g_{s} \sigma G q\right\rangle^{2}\langle\bar{q} q\rangle^{2} \int_{y_{i}}^{y_{f}} d y e^{-\frac{\tilde{m}^{2}}{T^{2}}}\{(y-1) y\}\left\{-\frac{1}{192 \pi^{2}}-\frac{\tilde{m}^{6}}{1152 \pi^{2} T^{6}}-\frac{\tilde{m}^{4}}{384 \pi^{2} T^{4}}-\frac{\tilde{m}^{2}}{192 \pi^{2} T^{2}}\right\} \\
& +\left\langle\bar{q} g_{s} \sigma G q\right\rangle^{2}\langle\bar{q} q\rangle^{2} \int_{y_{i}}^{y_{f}} d y e^{-\frac{\tilde{m}^{2}}{T^{2}}}\{(y-1) y\}\left\{\frac{1}{192 \pi^{2}}-\frac{\tilde{m}^{6}}{576 \pi^{2} T^{6}}-\frac{\tilde{m}^{4}}{384 \pi^{2} T^{4}}\right\} \\
& +m_{c}^{2}\left\langle\bar{q} g_{s} \sigma G q\right\rangle^{2}\langle\bar{q} q\rangle^{2} \int_{y_{i}}^{y_{f}} d y e^{-\frac{\tilde{m}^{2}}{T^{2}}}\left\{\frac{\tilde{m}^{4}}{64 \pi^{2} T^{6}}\right\} \\
& +\langle\bar{q} q\rangle^{2}\left\langle\bar{q} g_{s} \sigma G q\right\rangle^{2} \int_{y_{i}}^{y_{f}} d y e^{-\frac{\tilde{m}^{2}}{T^{2}}}\{(y-1) y\}\left\{\frac{1}{31104 \pi^{4}}-\frac{\tilde{m}^{6}}{93312 \pi^{4} T^{6}}-\frac{\tilde{m}^{4}}{62208 \pi^{4} T^{4}}\right\} \\
& +m_{c}^{2}\langle\bar{q} q\rangle^{2}\left\langle\bar{q} g_{s} \sigma G q\right\rangle^{2} \int_{y_{i}}^{y_{f}} d y e^{-\frac{\tilde{m}^{2}}{T^{2}}}\left\{\frac{\tilde{m}^{4}}{62208 \pi^{4} T^{6}}\right\} \\
& +\langle\bar{q} q\rangle^{2}\left\langle\bar{q} g_{s} \sigma G q\right\rangle^{2} \int_{y_{i}}^{y_{f}} d y e^{-\frac{\tilde{m}^{2}}{T^{2}}}\{(y-1) y\}\left\{-\frac{1}{31104 \pi^{4}}-\frac{\tilde{m}^{6}}{186624 \pi^{4} T^{6}}-\frac{\tilde{m}^{4}}{62208 \pi^{4} T^{4}}-\frac{\tilde{m}^{2}}{31104 \pi^{4} T^{2}}\right\}
\end{aligned}
$$

$$
\begin{aligned}
\mathbf{S}_{C}(16)_{2}= & m_{c}^{2}\langle\bar{q} q\rangle^{4}\left\langle g_{s}^{2} G G\right\rangle \int_{y_{i}}^{y_{f}} d y e^{-\frac{\tilde{m}^{2}}{T^{2}}}\left\{\frac{y-1}{y^{2}}\right\}\left\{\frac{\tilde{m}^{2}}{7776 \pi^{2} T^{4}}-\frac{1}{5184 \pi^{2} T^{2}}\right\} \\
& +m_{c}^{2}\langle\bar{q} q\rangle^{4}\left\langle g_{s}^{2} G G\right\rangle \int_{y_{i}}^{y_{f}} d y e^{-\frac{\tilde{m}^{2}}{T^{2}}}\left\{\frac{1}{y^{2}}\right\}\left\{\frac{1}{1728 \pi^{2} T^{2}}\right\} \\
& +m_{c}^{4}\langle\bar{q} q\rangle^{4}\left\langle g_{s}^{2} G G\right\rangle \int_{y_{i}}^{y_{f}} d y e^{-\frac{\tilde{m}^{2}}{T^{2}}}\left\{\frac{1}{y^{3}}\right\}\left\{-\frac{1}{5184 \pi^{2} T^{4}}\right\} \\
& +m_{c}^{2}\left\langle g_{s}^{2} G G\right\rangle\langle\bar{q} q\rangle^{4} \int_{y_{i}}^{y_{f}} d y e^{-\frac{\tilde{m}^{2}}{T^{2}}}\left\{\frac{y-1}{y^{2}}\right\}\left\{\frac{\tilde{m}^{2}}{15552 \pi^{2} T^{4}}\right\} \\
& +\langle\bar{q} q\rangle^{4}\left\langle g_{s}^{2} G G\right\rangle \int_{y_{i}}^{y_{f}} d y e^{-\frac{\tilde{m}^{2}}{T^{2}}}\{(y-1) y\}\left\{\frac{1}{2592 \pi^{2}}-\frac{\tilde{m}^{6}}{7776 \pi^{2} T^{6}}-\frac{\tilde{m}^{4}}{5184 \pi^{2} T^{4}}\right\} \\
& +m_{c}^{2}\langle\bar{q} q\rangle^{4}\left\langle g_{s}^{2} G G\right\rangle \int_{y_{i}}^{y_{f}} d y e^{-\frac{\tilde{m}^{2}}{T^{2}}}\left\{\frac{\tilde{m}^{4}}{5184 \pi^{2} T^{6}}\right\} \\
& +\langle\bar{q} q\rangle^{4}\left\langle g_{s}^{2} G G\right\rangle \int_{y_{i}}^{y_{f}} d y e^{-\frac{\tilde{m}^{2}}{T^{2}}}\{(y-1) y\}\left\{-\frac{1}{2592 \pi^{2}}-\frac{\tilde{m}^{6}}{15552 \pi^{2} T^{6}}-\frac{\tilde{m}^{4}}{5184 \pi^{2} T^{4}}-\frac{\tilde{m}^{2}}{2592 \pi^{2} T^{2}}\right\} .
\end{aligned}
$$

Type D for $J_{4}$ 


$$
\begin{aligned}
\mathbf{S}_{D}(12) & =m_{c}^{2}\langle\bar{q} q\rangle^{4} \int_{y_{i}}^{y_{f}} d y \int_{z_{i}}^{1-y} d z e^{-\frac{\bar{m}^{2}}{T^{2}}}\left\{\frac{y+z-1}{y}\right\}\left\{\frac{\bar{m}^{2}}{2592 \pi^{4}}\right\} \\
& +m_{c}^{2}\langle\bar{q} q\rangle^{4} \int_{y_{i}}^{y_{f}} d y \int_{z_{i}}^{1-y} d z e^{-\frac{\bar{m}^{2}}{T^{2}}}\left\{-\frac{z(y+z-1)}{y^{2}}\right\}\left\{-\frac{\bar{m}^{2}}{7776 \pi^{4}}\right\} \\
& +m_{c}^{2}\langle\bar{q} q\rangle^{4} \int_{y_{i}}^{y_{f}} d y \int_{z_{i}}^{1-y} d z e^{-\frac{\bar{m}^{2}}{T^{2}}}\left\{-\frac{z(y+z-1)}{y^{2}}\right\}\left\{-\frac{\bar{m}^{2}}{3888 \pi^{4}}\right\} \\
& +m_{c}^{2}\langle\bar{q} q\rangle^{4} \int_{y_{i}}^{y_{f}} d y \int_{z_{i}}^{1-y} d z e^{-\frac{\bar{m}^{2}}{T^{2}}}\left\{-\frac{z(y+z-1)}{y^{2}}\right\}\left\{\frac{\bar{m}^{2}}{11664 \pi^{4}}\right\} \\
& +m_{c}^{2}\langle\bar{q} q\rangle^{4} \int_{y_{i}}^{y_{f}} d y \int_{z_{i}}^{1-y} d z e^{-\frac{\bar{m}^{2}}{T^{2}}}\left\{-\frac{z(y+z-1)}{y^{2}}\right\}\left\{\frac{\bar{m}^{2}}{5832 \pi^{4}}\right\} \\
& +\langle\bar{q} q\rangle^{4} \int_{y_{i}}^{y_{f}} d y \int_{z_{i}}^{1-y} d z e^{-\frac{\bar{m}^{2}}{T^{2}}}\left\{-\frac{z(y+z-1)}{y}\right\}\left\{-\frac{\bar{m}^{4} y}{11664 \pi^{4}}\right\} \\
& +\langle\bar{q} q\rangle^{4} \int_{y_{i}}^{y_{f}} d y \int_{z_{i}}^{1-y} d z e^{-\frac{\bar{m}^{2}}{T^{2}}}\left\{-\frac{z(y+z-1)}{y}\right\}\left\{-\frac{\bar{m}^{4} y}{5832 \pi^{4}}\right\}
\end{aligned}
$$

